\title{
Updated 34-band Photometry for the SINGS/KINGFISH Samples of Nearby Galaxies
}

D. A. Dale ${ }^{1}$, D. O. Cook $^{2}$, H. Roussel ${ }^{3}$, J. A. Turner ${ }^{1}$, L. Armus ${ }^{4}$, A. D. Bolatto ${ }^{5}$, M. Boquien ${ }^{6}$, M. J. I. Brown ${ }^{7}$, D. Calzetti $^{8}$, I. De Looze ${ }^{9}$, M. Galametz ${ }^{10,11}$, K. D. Gordon ${ }^{12}$, B. A. Groves ${ }^{13}$, T. H. Jarrett ${ }^{14}$, G. Helou ${ }^{4}$, R. Herrera-Camus ${ }^{15}$, J. L. Hinz ${ }^{16}$, L. K. Hunt ${ }^{17}$, R. C. Kennicutt ${ }^{18}$, E. J. Murphy ${ }^{19}$, A. Rest ${ }^{12}$, K. M. Sandstrom ${ }^{20}$, J.-D. T. Smith ${ }^{21}$, F. S. Tabatabaei ${ }^{22}$, and C. D. Wilson ${ }^{23}$

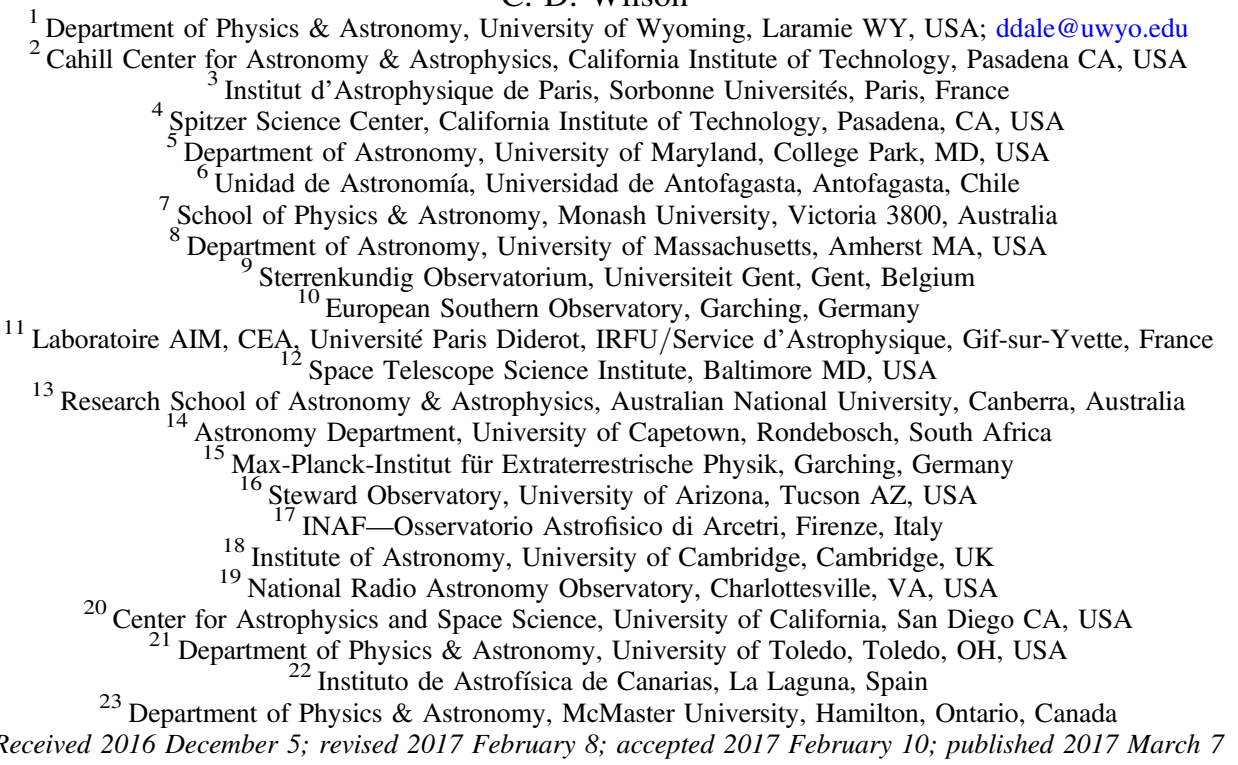

\begin{abstract}
We present an update to the ultraviolet-to-radio database of global broadband photometry for the 79 nearby galaxies that comprise the union of the KINGFISH (Key Insights on Nearby Galaxies: A Far-Infrared Survey with Herschel) and SINGS (Spitzer Infrared Nearby Galaxies Survey) samples. The 34-band data set presented here includes contributions from observational work carried out with a variety of facilities including GALEX, SDSS, Pan-STARRS1, NOAO, 2MASS, Wide-Field Infrared Survey Explorer, Spitzer, Herschel, Planck, JCMT, and the VLA. Improvements of note include recalibrations of previously published SINGS $B V R_{\mathrm{C}} I_{\mathrm{C}}$ and KINGFISH farinfrared/submillimeter photometry. Similar to previous results in the literature, an excess of submillimeter emission above model predictions is seen primarily for low-metallicity dwarf or irregular galaxies. This 33-band photometric data set for the combined KINGFISH+SINGS sample serves as an important multiwavelength reference for the variety of galaxies observed at low redshift. A thorough analysis of the observed spectral energy distributions is carried out in a companion paper.
\end{abstract}

Key words: galaxies: photometry - galaxies: ISM - infrared: ISM - ISM: general

Supporting material: extended figure

\section{Introduction}

Access to panchromatic broadband photometry for galaxies is crucial to fully understanding the characteristics of, and relative contributions to, galaxy spectra from the various processes related to interstellar attenuation, star formation, and the feeding of supermassive black holes (Silva et al. 1998; da Cunha et al. 2008; M. Boquien 2016, in preparation). Although a fairly complete multiwavelength data set has been published for the SINGS (Spitzer Infrared Nearby Galaxies Survey) sample of 75 nearby galaxies (Kennicutt et al. 2003; Dale et al. 2005, 2007), subsequent far-infrared/submillimeter Herschel broadband data were later published for the KINGFISH (Key Insights on Nearby Galaxies: A Far-Infrared Survey with Herschel) sample of 61 nearby galaxies (Kennicutt et al. 2011; Dale et al. 2012), a sample for which 57 of the 61 targets are also SINGS targets. The photometric data sets from the combined SINGS/KINGFISH surveys have served as important references for studies seeking to understand the diverse properties of galaxies in the Local Universe (da Cunha et al. 2008; Noll et al. 2009; Jonsson et al. 2010; Rémy-Ruyer et al. 2015), or to serve as redshift-zero comparison samples to higher redshift galaxies (Kartaltepe et al. 2010; Maiolino et al. 2015; Scoville et al. 2016).

In this effort we present an update to the global (spatially integrated) photometry for the 79 nearby galaxies that comprise the union of the KINGFISH and SINGS samples. This update includes a recalibration of the Dale et al. (2012) KINGFISH far-infrared/submillimeter photometry, necessary since the calibration of the Herschel photometers has undergone multiple revisions since those data were first published. We also carry out a Pan-STARRS1-based recalibration of the $B V R_{\mathrm{C}} I_{\mathrm{C}}$ fluxes previously published in Dale et al. (2007). A portion of these 
ground-based broadband optical data is suspect, since the data were originally taken in non-photometric conditions, and the ensuing attempts to calibrate the non-photometric frames were insufficient. We also include new ugriz and $12 \mu \mathrm{m}$ photometry from the Sloan Digital Sky Survey (SDSS) and the Wide-Field Infrared Survey Explorer (WISE) mission, respectively. In addition, the Herschel PACS photometry for KINGFISH galaxy NGC 0584 was not included in Dale et al. (2012) since these imaging data were not yet taken. Furthermore, we include new Herschel photometry for six SINGS galaxies from the Herschel Very Nearby Galaxy Survey (PI C. Wilson) that are not in the KINGFISH sample (NGC 2403, M81 = NGC 3031, M82 = NGC 3034, NGC 4125, M51a = NGC 5194, and M51b = NGC 5195). Finally, we complete the presentation of a multiwavelength database by including previously published global photometry from ultraviolet (GALEX), infrared/submillimeter (2MASS, Spitzer, SCUBA), and radio (VLA) wavelengths.

Section 2 provides a brief overview of the galaxy sample, Section 3 recapitulates the relevant observations and approaches to data processing, Section 4 describes the salient results, and Section 5 provides the summary. The companion paper (L.K. Hunt et al. 2017, in preparation) explores the observed spectral energy distributions (SEDs) by using and comparing fits employing three popular fitting tools: GRASIL, MAGPHYS, and CIGALE (Silva et al. 1998; da Cunha et al. 2008; Noll et al. 2009).

\section{Galaxy Sample}

Table 1 presents the full list of 79 galaxies that form the union of the SINGS and KINGFISH samples. The sample was chosen to be a representative sampling of the Local Universe; the sample is not volume limited and thus does not represent a statistical sampling of the Local Volume, but the sample is representative of the diversity of local galaxies. These galaxies were selected to span a range of morphologies, colors, and luminosities (e.g., Figures 5 and 3 of Kennicutt et al. 2003; Dale et al. 2012, respectively). Figure 1 demonstrates the sample's range of optical colors and near-infrared luminosities; a few galaxies reside in the red sequence near the top of the diagram, but most of the sample spans the blue star-forming sequence. The sample is comprised of $8 \%, 11 \%, 63 \%$, and $18 \%$ early-type, lenticular, spiral, and irregular galaxies, respectively, based on the optical morphologies provided in the NASA/IPAC Extragalactic Database (NED). This galaxy sample has no sources for which the optical luminosity is dominated by AGN emission, although one-third have signatures of Seyfert/LINER nuclei (Tajer et al. 2005; Dale et al. 2006; Moustakas et al. 2010; Grier et al. 2011). There are only a few galaxies that are undoubtedly interacting with neighboring galaxies, including NGC 5194 (with NGC 5195), NGC 1097 (with NGC 1097A), NGC 1316 (with NGC 1317), and NGC 3190 (with NGC 3187). The distances reach out to $\sim 30 \mathrm{Mpc}$ with a median value of $\sim 10 \mathrm{Mpc}$.

\section{Observations and Data Processing}

Much of the photometry presented here has already been described in Dale et al. (2007, 2012), therefore we focus the following discussion on important differences from what is presented in those publications. The imaging bandpasses used here are listed in the heading of Table 2. The central wavelengths and widths of the filters are computed via

$$
\bar{\lambda} \equiv \frac{\int \lambda \mathcal{T}(\lambda) d \lambda}{\int \mathcal{T}(\lambda) d \lambda}
$$

and

$$
\Delta \equiv \int \mathcal{T}(\lambda) d \lambda
$$

where $\mathcal{T}$ represents the filter transmittance normalized to peak at unity, based on the filter profiles compiled by Noll et al. (2009) for use in the CIGALE software package (Noll et al. 2009).

\subsection{Ground-based Optical}

Some of the optical $B V R_{\mathrm{C}} I_{\mathrm{C}}$ photometry from Dale et al. (2007) suffers from faulty calibration (Muñoz-Mateos et al. 2009). Sometimes insufficient numbers of standard star observations were taken, sometimes the standards were saturated, and some of the frames taken in non-photometric conditions were not successfully calibrated a posteriori. A recalibration is carried out here via comparison of photometry on foreground stars in Pan-STARRS1 (PS1) $g_{\mathrm{PS} 1} r_{\mathrm{PS} 1} i_{\mathrm{PS} 1} z_{\mathrm{PS} 1}$ and in our 2007-era $B V R_{\mathrm{C}} I_{\mathrm{C}}$ imaging. The PS1 $3 \pi$ Survey uses the $1.8 \mathrm{~m}$ telescope on Mount Haleakala to map the sky north of $\delta=-30^{\circ}$ with multiple passes of 30-60 s exposures in each of their five SDSS-like filters (Schlafly et al. 2012; Magnier et al. 2013).

Care was taken in the comparison to only use bright $\left(r_{\mathrm{PS} 1}<19 \mathrm{mag}\right)$, unsaturated sources with point-spread functions (PSFs) that agree with the seeing profiles of each image (i.e., background galaxies are excluded). The median number of foreground stars per galaxy used for this purpose was 15 . Aperture diameters for the foreground stellar photometry were typically 7 "; increasing the aperture diameter by $50 \%$ results in a $<1 \%$ difference in the calibration. The PS1 fluxes were converted from their measured values at 1.2 airmasses into 0 airmasses (Table 4 of Tonry et al. 2012), and the small (tens of millimag) corrections suggested by Scolnic et al. (2015) were incorporated (see their Table 3); the small calibration modifications of Scolnic et al. (2015) are based on a "super calibration" that combines flux measurements of secondary standards from several surveys including PS1 and SDSS.

We adopted the Tonry et al. (2012) quadratic filter transformations for stars between Pan-STARRS1 $g_{\mathrm{PS} 1} r_{\mathrm{PS} 1} i_{\mathrm{PS} 1} z_{\mathrm{PS} 1}$ and $B V R_{\mathrm{C}} I_{\mathrm{C}}$, although very similar results are obtained when using linear transformations between SDSS and Johnson/Cousins filters (Jester et al. 2005; Lupton et al. 2005; Jordi et al. 2006; Chonis \& Gaskell 2008; Tonry et al. 2012). The only significant outliers for any of these various stellar transformations are the Chonis \& Gaskell (2008) $V$ and Lupton et al. (2005) $R_{\mathrm{C}}$ linear transformations, both of which yield calibrations that ultimately result in galaxy fluxes that are $25 \%-30 \% \quad(\sim 0.25-0.30 \mathrm{mag})$ brighter compared to when using other published transformations. The photometric calibrations are derived in practice from the errorweighted differences between the instrumental $B V R_{\mathrm{C}} I_{\mathrm{C}}$ fluxes and the measured PS1 fluxes (transformed to $B V R_{\mathrm{C}} I_{\mathrm{C}}$ ) for the suite of suitable foreground stars identified for each galaxy. The dispersions in these bootstrap calibrations range from $2 \%$ to $15 \%$ (with a median of $5 \%$ ) and contribute to the overall photometric uncertainty estimates; the photometric uncertainties for the PS1-recalibrated fluxes are the sums in quadrature of these dispersions along with the uncertainties in the published 
Table 1

Galaxy Sample

\begin{tabular}{|c|c|c|c|c|c|c|c|c|c|}
\hline Galaxy & $\begin{array}{l}\text { Alternative } \\
\text { Name }\end{array}$ & $\begin{array}{l}\text { Optical } \\
\text { Morph. }\end{array}$ & $\begin{array}{c}E(B-V) \\
\quad(\text { mag. })\end{array}$ & $\begin{array}{c}\alpha_{0} \text { and } \delta_{0} \\
(\mathrm{~J} 2000)\end{array}$ & $\begin{array}{l}2 a \\
\left({ }^{\prime \prime}\right)\end{array}$ & $\begin{array}{l}2 b \\
(")\end{array}$ & $\begin{array}{l}\text { PA } \\
\left({ }^{\circ}\right)\end{array}$ & $\begin{array}{c}D \\
(\mathrm{Mpc})\end{array}$ & $\begin{array}{l}\text { TIR } \\
\left(L_{\odot}\right)\end{array}$ \\
\hline NGC 0024 & UGC A002 & SAc & 0.017 & $000955.9-245755$ & 301 & 216 & 45 & 8.20 & 8.8 \\
\hline NGC 0337 & NGC 0337 & SBd & 0.096 & $005950.7-073444$ & 253 & 194 & 140 & 19.30 & 10.1 \\
\hline NGC 0584 & NGC 0584 & $\mathrm{E} 4$ & 0.036 & $013120.6-065205$ & 326 & 278 & 60 & 20.80 & 8.7 \\
\hline NGC 0628 & Messier 074 & SAc & 0.060 & $013642.4+154711$ & 879 & 808 & 90 & 7.20 & 9.9 \\
\hline NGC 0855 & UGC 01718 & $\mathrm{E}$ & 0.061 & $021403.7+275237$ & 259 & 169 & 60 & 9.73 & 8.6 \\
\hline NGC 0925 & UGC 01913 & SABd & 0.065 & $022713.6+333504$ & 735 & 486 & 105 & 9.12 & 9.7 \\
\hline NGC 1097 & UGC A041 & $\mathrm{SBb}$ & 0.023 & $024618.0-301642$ & 758 & 612 & 130 & 14.20 & 10.6 \\
\hline NGC 1266 & NGC 1266 & SB0 & 0.085 & $031600.6-022541$ & 234 & 232 & 0 & 30.60 & 10.4 \\
\hline NGC 1291 & NGC 1291 & $\mathrm{SB} 0 / \mathrm{a}$ & 0.011 & $031717.9-410616$ & 884 & 836 & 90 & 10.40 & 9.5 \\
\hline NGC 1316 & FornaxA & $\mathrm{SAB} 0$ & 0.018 & $032241.2-371210$ & 864 & 583 & 50 & 21.00 & 9.9 \\
\hline NGC 1377 & NGC 1377 & So & 0.024 & $033639.0-205408$ & 181 & 162 & 90 & 24.60 & 10.1 \\
\hline NGC 1404 & NGC 1404 & E1 & 0.010 & $033852.3-353540$ & 524 & 369 & 149 & 20.20 & $\ldots$ \\
\hline IC0342 & UGC 02847 & SABcd & 0.480 & $034659.5+680539$ & 1667 & 1439 & 100 & 3.28 & 10.1 \\
\hline NGC 1482 & NGC 1482 & SA0 & 0.034 & $035439.0-203009$ & 349 & 310 & 119 & 22.60 & 10.6 \\
\hline NGC 1512 & NGC 1512 & SBab & 0.009 & $040355.6-432149$ & 1001 & 928 & 83 & 11.60 & 9.5 \\
\hline NGC 1566 & & SABbc & 0.008 & $042000.4-545615$ & 552 & 435 & 40 & 18.00 & 10.6 \\
\hline NGC 1705 & & SA0 & 0.007 & $045413.5-532137$ & 167 & 120 & 40 & 5.80 & 8.0 \\
\hline NGC 2146 & UGC 03429 & Sbab & 0.082 & $061835.6+782129$ & 236 & 235 & 120 & 17.20 & 11.0 \\
\hline NGC 2403 & UGC 03918 & SABcd & 0.034 & $073655.1+653534$ & 1512 & 929 & 124 & 3.50 & 9.6 \\
\hline HolmbergII & UGC 04305 & $\mathrm{Im}$ & 0.027 & $081910.8+704320$ & 554 & 465 & 60 & 3.05 & 7.8 \\
\hline M081DwarfA & & $\mathrm{I} ?$ & 0.018 & $082356.0+710145$ & 78 & 78 & 90 & 3.50 & $\ldots$ \\
\hline DDO 053 & UGC 04459 & $\operatorname{Im}$ & 0.033 & $083407.4+661043$ & 155 & 142 & 90 & 3.61 & 7.0 \\
\hline NGC 2798 & UGC 04905 & $\mathrm{SBa}$ & 0.017 & $091723.1+415957$ & 235 & 232 & 90 & 25.80 & 10.6 \\
\hline NGC 2841 & UGC 04966 & $\mathrm{SAb}$ & 0.013 & $092203.3+505837$ & 629 & 334 & 150 & 14.10 & 10.1 \\
\hline NGC 2915 & & I0 & 0.236 & $092609.4-763736$ & 183 & 132 & 110 & 3.78 & 7.6 \\
\hline HolmbergI & UGC 05139 & $\mathrm{IABm}$ & 0.044 & $094033.6+711120$ & 264 & 219 & 63 & 3.90 & 7.1 \\
\hline NGC 2976 & UGC 05221 & SAc & 0.062 & $094715.3+675509$ & 541 & 353 & 144 & 3.55 & 8.9 \\
\hline NGC 3049 & UGC 05325 & SBab & 0.033 & $095449.6+091614$ & 218 & 160 & 29 & 19.20 & 9.5 \\
\hline NGC 3031 & Messier 081 & SAab & 0.069 & $095531.8+690403$ & 1628 & 1122 & 154 & 3.50 & 9.6 \\
\hline NGC 3034 & Messier 082 & I0 & 0.134 & $095552.1+694057$ & 698 & 581 & 65 & 3.50 & 10.8 \\
\hline HolmbergIX & UGC 05336 & $\mathrm{Im}$ & 0.068 & $095729.2+690250$ & 247 & 180 & 40 & 3.50 & $\cdots$ \\
\hline NGC 3077 & UGC 05398 & IOpec & 0.058 & $100317.5+684354$ & 488 & 436 & 64 & 3.83 & 8.9 \\
\hline M081DwarfB & UGC 05423 & $\mathrm{Im}$ & 0.068 & $100531.2+702151$ & 134 & 90 & 139 & 3.60 & 6.5 \\
\hline NGC 3190 & UGC 05559 & SAap & 0.022 & $101805.7+214957$ & 334 & 196 & 117 & 19.30 & 9.8 \\
\hline NGC 3184 & UGC 05557 & SABcd & 0.014 & $101815.6+412542$ & 614 & 538 & 169 & 11.70 & 10.0 \\
\hline NGC 3198 & UGC 05572 & $\mathrm{SBc}$ & 0.011 & $101954.8+453301$ & 518 & 315 & 35 & 14.10 & 10.0 \\
\hline IC2574 & UGC 05666 & $\mathrm{SABm}$ & 0.031 & $102823.9+682505$ & 864 & 486 & 59 & 3.79 & 8.3 \\
\hline NGC 3265 & UGC 05705 & $\mathrm{E}$ & 0.021 & $103106.8+284751$ & 184 & 175 & 50 & 19.60 & 9.4 \\
\hline Mrk 33 & UGC 05720 & $\mathrm{Im}$ & 0.010 & $103231.2+542359$ & 181 & 177 & 90 & 21.70 & 9.8 \\
\hline NGC 3351 & Messier 095 & $\mathrm{SBb}$ & 0.024 & $104358.1+114210$ & 592 & 441 & 11 & 9.33 & 9.9 \\
\hline NGC 3521 & UGC 06150 & $\mathrm{SABbc}$ & 0.049 & $110548.1-000127$ & 926 & 455 & 165 & 11.20 & 10.5 \\
\hline NGC 3621 & UGC A232 & SAd & 0.069 & $111818.3-324855$ & 791 & 555 & 160 & 6.55 & 9.9 \\
\hline NGC 3627 & Messier 066 & $\mathrm{SABb}$ & 0.029 & $112013.4+125927$ & 745 & 486 & 167 & 9.38 & 10.4 \\
\hline NGC 3773 & UGC 06605 & SA0 & 0.023 & $113813.1+120644$ & 118 & 116 & 0 & 12.40 & 8.8 \\
\hline NGC 3938 & UGC 06856 & SAc & 0.018 & $115250.3+440715$ & 504 & 468 & 0 & 17.90 & 10.3 \\
\hline NGC 4125 & UGC 07118 & E6p & 0.016 & $120805.8+651024$ & 228 & 151 & 90 & 21.40 & 9.1 \\
\hline NGC 4236 & UGC 07306 & SBdm & 0.013 & $121643.2+692719$ & 1240 & 369 & 162 & 4.45 & 8.7 \\
\hline NGC 4254 & Messier 099 & SAc & 0.033 & $121849.7+142519$ & 519 & 420 & 60 & 14.40 & 10.6 \\
\hline NGC 4321 & Messier 100 & $\mathrm{SABbc}$ & 0.023 & $122254.8+154907$ & 558 & 483 & 40 & 14.30 & 10.5 \\
\hline NGC 4450 & UGC 07594 & SAab & 0.024 & $122830.1+170454$ & 401 & 284 & 180 & 20.00 & 9.9 \\
\hline NGC 4536 & UGC 07732 & $\mathrm{SABbc}$ & 0.016 & $123427.5+021113$ & 454 & 376 & 120 & 14.50 & 10.3 \\
\hline NGC 4552 & Messier 089 & $\mathrm{E}$ & 0.035 & $123539.8+123323$ & 306 & 306 & 90 & 4.90 & 7.7 \\
\hline NGC 4559 & UGC 07766 & SABcd & 0.015 & $123558.1+275752$ & 576 & 327 & 140 & 6.98 & 9.5 \\
\hline NGC 4569 & Messier 090 & SABab & 0.040 & $123650.2+131001$ & 593 & 327 & 21 & 9.86 & 9.7 \\
\hline NGC 4579 & Messier 058 & $\mathrm{SABb}$ & 0.035 & $123743.8+114858$ & 325 & 271 & 90 & 16.40 & 10.1 \\
\hline NGC 4594 & Messier 104 & SAa & 0.044 & $123959.6-113726$ & 767 & 669 & 90 & 9.08 & 9.5 \\
\hline NGC 4625 & UGC 07861 & SABmp & 0.016 & $124154.8+411623$ & 298 & 214 & 100 & 9.30 & 8.8 \\
\hline NGC 4631 & UGC 07865 & SBd & 0.015 & $124204.2+323219$ & 901 & 240 & 85 & 7.62 & 10.4 \\
\hline NGC 4725 & UGC 07989 & SABab & 0.010 & $125027.7+252948$ & 689 & 523 & 30 & 11.90 & 9.9 \\
\hline NGC 4736 & Messier 094 & SAab & 0.015 & $125055.2+410652$ & 944 & 899 & 0 & 4.66 & 9.8 \\
\hline DDO 154 & UGC 08024 & $\mathrm{IBm}$ & 0.008 & $125407.6+270916$ & 216 & 126 & 50 & 4.30 & $\cdots$ \\
\hline NGC 4826 & Messier 064 & SAab & 0.036 & $125643.3+214048$ & 716 & 427 & 114 & 5.27 & 9.6 \\
\hline DDO 165 & UGC 08201 & $\mathrm{Im}$ & 0.021 & $130625.9+674229$ & 263 & 161 & 90 & 4.57 & $\ldots$ \\
\hline
\end{tabular}


Table 1

(Continued)

\begin{tabular}{|c|c|c|c|c|c|c|c|c|c|}
\hline Galaxy & $\begin{array}{l}\text { Alternative } \\
\text { Name }\end{array}$ & $\begin{array}{l}\text { Optical } \\
\text { Morph. }\end{array}$ & $\begin{array}{c}E(B-V) \\
\quad(\text { mag. })\end{array}$ & $\begin{array}{c}\alpha_{0} \text { and } \delta_{0} \\
(\mathbf{J} 2000)\end{array}$ & $\begin{array}{l}2 a \\
(")\end{array}$ & $\begin{array}{l}2 b \\
(")\end{array}$ & $\begin{array}{l}\text { PA } \\
\left({ }^{\circ}\right)\end{array}$ & $\begin{array}{c}D \\
(\mathrm{Mpc})\end{array}$ & $\begin{array}{l}\text { TIR } \\
\left(L_{\odot}\right)\end{array}$ \\
\hline NGC 5033 & UGC 08307 & SAc & 0.010 & $131328.2+363534$ & 729 & 467 & 180 & 13.30 & 10.3 \\
\hline NGC 5055 & Messier 063 & SAbc & 0.015 & $131549.2+420147$ & 1097 & 711 & 80 & 7.94 & 10.3 \\
\hline NGC 5194 & Messier 051a & $\mathrm{SABbc}$ & 0.030 & $132950.6+471307$ & 1699 & 1129 & 15 & 8.20 & 10.6 \\
\hline NGC 5195 & Messier 051b & SB0p & 0.031 & $132959.4+471556$ & 202 & 191 & 0 & 8.20 & 9.3 \\
\hline NGC 5398 & Tololo89 & SBdm & 0.056 & $140121.2-330402$ & 198 & 146 & 0 & 7.66 & 8.6 \\
\hline NGC 5457 & Messier 101 & SABcd & 0.007 & $140325.0+542429$ & 1800 & 1446 & 37 & 6.70 & 10.3 \\
\hline NGC 5408 & & $\mathrm{IBm}$ & 0.059 & $140321.1-412241$ & 256 & 209 & 67 & 4.80 & 8.3 \\
\hline NGC 5474 & UGC 09013 & SAcd & 0.009 & $140500.8+533920$ & 412 & 373 & 90 & 6.80 & 8.7 \\
\hline NGC 5713 & UGC 09451 & SABbcp & 0.034 & $144011.4-001726$ & 225 & 225 & 90 & 21.40 & 10.5 \\
\hline NGC 5866 & UGC 09723 & So & 0.012 & $150628.8+554551$ & 500 & 306 & 129 & 15.30 & 9.7 \\
\hline IC4710 & & $\mathrm{SBm}$ & 0.076 & $182838.9-665903$ & 313 & 219 & 120 & 8.50 & 8.6 \\
\hline NGC 6822 & DDO 229 & $\mathrm{IBm}$ & 0.199 & $194453.2-144811$ & 1453 & 1100 & 150 & 0.60 & 7.9 \\
\hline NGC 6946 & UGC 11597 & SABcd & 0.294 & $203449.2+600959$ & 953 & 928 & 0 & 6.80 & 10.5 \\
\hline NGC 7331 & UGC 12113 & $\mathrm{SAb}$ & 0.078 & $223704.3+342435$ & 683 & 335 & 168 & 14.50 & 10.7 \\
\hline NGC 7552 & IC5294 & SAc & 0.012 & $231610.8-423505$ & 441 & 325 & 120 & 22.30 & 11.0 \\
\hline NGC 7793 & & SAd & 0.017 & $235749.9-323525$ & 716 & 526 & 98 & 3.91 & 9.3 \\
\hline
\end{tabular}

Note. Foreground extinctions derive from Schlafly \& Finkbeiner (2011). $2 a$ and $2 b$ are the lengths of the major and minor axes used in the elliptical aperture photometry described herein; the position angle of the aperture's major axis is measured east of north. The total infrared luminosity listed in the last column is derived from Equation (5) of Dale et al. (2014) and the Spitzer 8,24, 70, and $160 \mu \mathrm{m}$ fluxes in Table 2 and assumes the distances provided in this table. WISE $22 \mu \mathrm{m}$ and Herschel PACS 70 and $160 \mu \mathrm{m}$ fluxes are used for NGC 3034 since its MIPS data suffer from saturation.

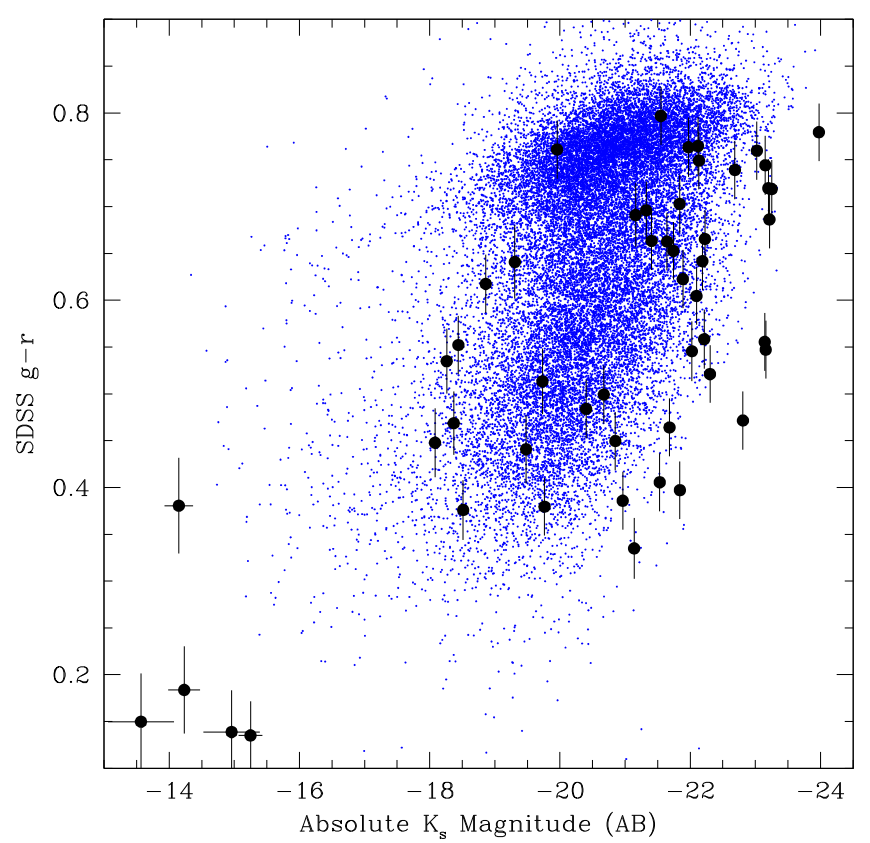

Figure 1. Comparison of global $g-r$ colors and absolute 2MASS $K_{\mathrm{s}}$ magnitudes for the KINGFISH/SINGS (large circles) and SDSS low-redshift samples $\left(10<d<150 \mathrm{Mpc}^{-1}\right.$; Blanton et al. 2005). The values are corrected for foreground Milky Way extinction.

$g_{\mathrm{PS} 1} r_{\mathrm{PS} 1} i_{\mathrm{PS} 1} z_{\mathrm{PS} 1} \rightarrow B V R_{\mathrm{C}} I_{\mathrm{C}}$ transformations and the instrumental galaxy flux measurements.

The Pan-STARRS1 survey does not encompass regions of the sky south of Galactic latitude $\delta=-30^{\circ}$, and thus PS1 calibration is not possible for 18 SINGS/KINGFISH objects. Table 3 indicates for which targets the $B V R_{\mathrm{C}} I_{\mathrm{C}}$ photometry has been recalibrated via PS1. Additional broadband optical photometry is possible via other ground-based efforts. SDSS ugriz imaging (Data Release 12) is used to provide optical fluxes for 51 of the 79 SINGS/KINGFISH galaxies. The union of the SDSS ugriz and PS1-recalibrated $B V R_{\mathrm{C}} I_{\mathrm{C}}$ samples comprises 63 galaxies, hence $80 \%$ of the full sample. The fraction of the sample for which we have reliable optical photometry approaches $100 \%$ after inclusion of $B V R_{\mathrm{C}} I_{\mathrm{C}}$ photometry from other global photometric data sets (see Table 3 and de Vaucouleurs et al. 1991; Muñoz-Mateos et al. 2009; Tully et al. 2009; Cook et al. 2014).

Other differences between our $B V R_{\mathrm{C}} I_{\mathrm{C}}$ photometry and those appearing in Dale et al. (2007) include the use of SINGS Data Release 5 (DR5) images (DR2 imaging was used in the previous publication), more robust sky level determinations (i.e., a significantly larger number of sky pixels are now used$38 \%$ more on average), and a fresh take on the editing of foreground stars and background galaxies (see Section 3.5). In some instances the DR5 images are noticeably flatter than their DR2 counterparts (e.g., NGC $0628 V R_{\mathrm{C}} I_{\mathrm{C}}$ ). Otherwise, the data-processing procedures are essentially identical to those already described in Dale et al. (2007).

\subsection{Herschel Infrared}

Fluxes based on Herschel PACS and SPIRE imaging are presented here for 67 of the 79 SINGS/KINGFISH galaxies. The Herschel PACS and SPIRE imaging observations are described in Dale et al. (2012) for the 61 KINGFISH galaxies, except for the PACS observations of NGC 0584, which were taken too late to appear in that publication. Another minor difference from Dale et al. (2012) is that the PACS maps used here are deeper for five KINGFISH objects since we have now incorporated additional data from other observing programs: Holmberg II, IC 2574, NGC 2798, NGC 4236, and NGC 4631. Deeper observations allow for more robust measurements, e.g., the 70 and $100 \mu \mathrm{m}$ flux-to-uncertainty ratios for Holmberg II are a factor of two larger than published in Dale et al. (2012) (see Section 4). We also incorporate here new Herschel imaging observations for 6 SINGS galaxies from the Herschel Very Nearby Galaxy Survey (VNGS; PI C. Wilson) that are not in the KINGFISH sample: NGC 2403, M81 = NGC 3031, M82 = NGC 3034, NGC 4125, $\mathrm{M} 51 \mathrm{a}=\mathrm{NGC} 5194$, and M51b $=$ NGC 5195. The observing 
Table 2

Global Flux Densities in Janskys Corrected for neither Galactic nor Intrinsic Extinction

\begin{tabular}{|c|c|c|c|c|c|c|c|c|c|c|}
\hline & GALEX & GALEX & & & & & SDSS & SDSS & SDSS & SDSS \\
\hline Filter & FUV & NUV & $B$ & $V$ & $R_{\mathrm{C}}$ & $I_{\mathrm{C}}$ & $u$ & $g$ & $r$ & $i$ \\
\hline $\bar{\lambda}(\mu \mathrm{m})$ & 0.154 & 0.231 & 0.441 & 0.551 & 0.659 & 0.806 & 0.356 & 0.482 & 0.626 & 0.767 \\
\hline$\Delta \lambda(\mu \mathrm{m})$ & 0.025 & 0.073 & 0.096 & 0.089 & 0.159 & 0.150 & 0.056 & 0.127 & 0.133 & 0.135 \\
\hline$A_{\lambda} / A_{V}$ & 2.586 & 2.994 & 1.310 & 1.0 & 0.788 & 0.577 & 1.642 & 1.219 & 0.849 & 0.641 \\
\hline NGC 0024 & $772 \pm 121 \mathrm{E}-3$ & $981 \pm 149 \mathrm{E}-3$ & $817 \pm 021 \mathrm{E}-2$ & $108 \pm 002 \mathrm{E}-1$ & $138 \pm 007 \mathrm{E}-1$ & $183 \pm 007 \mathrm{E}-1$ & $\ldots$ & $\ldots$ & $\ldots$ & $\ldots$ \\
\hline NGC 0337 & $463 \pm 064 \mathrm{E}-3$ & $819 \pm 113 \mathrm{E}-3$ & $786 \pm 046 \mathrm{E}-2$ & $908 \pm 044 \mathrm{E}-2$ & $108 \pm 006 \mathrm{E}-1$ & $139 \pm 015 \mathrm{E}-1$ & $249 \pm 006 \mathrm{E}-2$ & $664 \pm 014 \mathrm{E}-2$ & $100 \pm 002 \mathrm{E}-1$ & $121 \pm 002 \mathrm{E}-1$ \\
\hline NGC 0584 & $274 \pm 039 \mathrm{E}-4$ & $146 \pm 020 \mathrm{E}-3$ & $130 \pm 014 \mathrm{E}-1$ & $264 \pm 020 \mathrm{E}-1$ & $346 \pm 039 \mathrm{E}-1$ & $519 \pm 048 \mathrm{E}-1$ & $292 \pm 007 \mathrm{E}-2$ & $143 \pm 002 \mathrm{E}-1$ & $296 \pm 006 \mathrm{E}-1$ & $433 \pm 008 \mathrm{E}-1$ \\
\hline NGC 0628 & $469 \pm 073 \mathrm{E}-2$ & $591 \pm 091 \mathrm{E}-2$ & $528 \pm 033 \mathrm{E}-1$ & $721 \pm 033 \mathrm{E}-1$ & $921 \pm 042 \mathrm{E}-1$ & $118 \pm 006 \mathrm{E}+0$ & $179 \pm 003 \mathrm{E}-1$ & $492 \pm 009 \mathrm{E}-1$ & $804 \pm 016 \mathrm{E}-1$ & $108 \pm 002 \mathrm{E}+0$ \\
\hline NGC 0855 & $920 \pm 144 \mathrm{E}-4$ & $178 \pm 027 \mathrm{E}-3$ & $204 \pm 020 \mathrm{E}-2$ & $335 \pm 033 \mathrm{E}-2$ & $457 \pm 012 \mathrm{E}-2$ & & $840 \pm 029 \mathrm{E}-3$ & $268 \pm 006 \mathrm{E}-2$ & $468 \pm 010 \mathrm{E}-2$ & $633 \pm 013 \mathrm{E}-2$ \\
\hline NGC 0925 & $293 \pm 040 \mathrm{E}-2$ & $357 \pm 049 \mathrm{E}-2$ & $265 \pm 013 \mathrm{E}-1$ & $456 \pm 123 \mathrm{E}-1$ & $465 \pm 039 \mathrm{E}-1$ & $601 \pm 049 \mathrm{E}-1$ & $\ldots$ & $\ldots$ & $\ldots$ & $\ldots$ \\
\hline NGC 1097 & $298 \pm 042 \mathrm{E}-2$ & $418 \pm 058 \mathrm{E}-2$ & $373 \pm 013 \mathrm{E}-1$ & $641 \pm 023 \mathrm{E}-1$ & $898 \pm 033 \mathrm{E}-1$ & $124 \pm 004 \mathrm{E}+0$ & $\ldots$ & $\ldots$ & $\ldots$ & $\ldots$ \\
\hline NGC 1266 & $024 \pm 003 \mathrm{E}-4$ & $141 \pm 019 \mathrm{E}-4$ & $146 \pm 010 \mathrm{E}-2$ & $289 \pm 015 \mathrm{E}-2$ & $404 \pm 031 \mathrm{E}-2$ & $638 \pm 047 \mathrm{E}-2$ & $\ldots$ & $\ldots$ & $\ldots$ & $\ldots$ \\
\hline NGC 1291 & $698 \pm 109 \mathrm{E}-3$ & $151 \pm 023 \mathrm{E}-2$ & $579 \pm 021 \mathrm{E}-1$ & $118 \pm 004 \mathrm{E}+0$ & $172 \pm 006 \mathrm{E}+0$ & $241 \pm 008 \mathrm{E}+0$ & $\ldots$ & $\ldots$ & $\ldots$ & $\ldots$ \\
\hline NGC 1316 & $268 \pm 037 \mathrm{E}-3$ & $142 \pm 019 \mathrm{E}-2$ & $782 \pm 028 \mathrm{E}-1$ & $155 \pm 005 \mathrm{E}+0$ & $226 \pm 008 \mathrm{E}+0$ & $290 \pm 010 \mathrm{E}+0$ & $\ldots$ & $\ldots$ & $\ldots$ & $\ldots$ \\
\hline NGC 1377 & & $\ldots$ & $210 \pm 011 \mathrm{E}-2$ & $350 \pm 014 \mathrm{E}-2$ & $453 \pm 032 \mathrm{E}-2$ & $732 \pm 023 \mathrm{E}-2$ & $\ldots$ & $\ldots$ & $\ldots$ & $\ldots$ \\
\hline NGC 1404 & $889 \pm 123 \mathrm{E}-4$ & $253 \pm 035 \mathrm{E}-3$ & $172 \pm 006 \mathrm{E}-1$ & $355 \pm 013 \mathrm{E}-1$ & $513 \pm 018 \mathrm{E}-1$ & $764 \pm 028 \mathrm{E}-1$ & $\ldots$ & $\ldots$ & $\ldots$ & $\ldots$ \\
\hline IC0342 & $233 \pm 034 \mathrm{E}-2$ & $513 \pm 077 \mathrm{E}-2$ & $975 \pm 134 \mathrm{E}-1$ & $\ldots$ & $\ldots$ & $\ldots$ & $\ldots$ & $\ldots$ & $\ldots$ & $\ldots$ \\
\hline NGC 1482 & $303 \pm 047 \mathrm{E}-4$ & $106 \pm 015 \mathrm{E}-3$ & $499 \pm 028 \mathrm{E}-2$ & $692 \pm 045 \mathrm{E}-2$ & $976 \pm 095 \mathrm{E}-2$ & $150 \pm 013 \mathrm{E}-1$ & $\ldots$ & $\ldots$ & $\ldots$ & $\ldots$ \\
\hline NGC 1512 & $158 \pm 024 \mathrm{E}-2$ & $165 \pm 028 \mathrm{E}-2$ & $146 \pm 005 \mathrm{E}-1$ & $252 \pm 009 \mathrm{E}-1$ & $351 \pm 012 \mathrm{E}-1$ & $489 \pm 018 \mathrm{E}-1$ & $\ldots$ & $\ldots$ & $\ldots$ & $\ldots$ \\
\hline NGC 1566 & $509 \pm 071 \mathrm{E}-2$ & $612 \pm 084 \mathrm{E}-2$ & $348 \pm 012 \mathrm{E}-1$ & $593 \pm 021 \mathrm{E}-1$ & $697 \pm 025 \mathrm{E}-1$ & $64 \pm 031 \mathrm{E}-1$ & $\ldots$ & $\ldots$ & $\ldots$ & $\ldots$ \\
\hline NGC 1705 & $158 \pm 024 \mathrm{E}-2$ & $148 \pm 022 \mathrm{E}-2$ & $267 \pm 009 \mathrm{E}-2$ & $366 \pm 013 \mathrm{E}-2$ & $399 \pm 014 \mathrm{E}-2$ & $537 \pm 019 \mathrm{E}-2$ & $\ldots$ & $\ldots$ & $\ldots$ & $\ldots$ \\
\hline NGC 2146 & $188 \pm 028 \mathrm{E}-3$ & $485 \pm 072 \mathrm{E}-3$ & $119 \pm 015 \mathrm{E}-1$ & $210 \pm 027 \mathrm{E}-1$ & $\ldots$ & $537 \pm 014 \mathrm{E}-1$ & $\ldots$ & $\ldots$ & $\ldots$ & $\ldots$ \\
\hline NGC 2403 & $185 \pm 029 \mathrm{E}-1$ & $220 \pm 034 \mathrm{E}-1$ & $129 \pm 012 \mathrm{E}+0$ & $205 \pm 029 \mathrm{E}+0$ & $227 \pm 008 \mathrm{E}+0$ & $301 \pm 007 \mathrm{E}+0$ & $751 \pm 015 \mathrm{E}-1$ & $153 \pm 003 \mathrm{E}+0$ & $225 \pm 004 \mathrm{E}+0$ & $287 \pm 005 \mathrm{E}+0$ \\
\hline HoII & $340 \pm 053 \mathrm{E}-2$ & $369 \pm 056 \mathrm{E}-2$ & $127 \pm 009 \mathrm{E}-1$ & $149 \pm 007 \mathrm{E}-1$ & $201 \pm 022 \mathrm{E}-1$ & $303 \pm 049 \mathrm{E}-1$ & $\ldots$ & $\ldots$ & $\ldots$ & $\ldots$ \\
\hline M81dwA & $418 \pm 057 \mathrm{E}-4$ & $488 \pm 067 \mathrm{E}-4$ & $100 \pm 040 \mathrm{E}-3$ & $900 \pm 400 \mathrm{E}-4$ & $110 \pm 040 \mathrm{E}-3$ & $140 \pm 060 \mathrm{E}-3$ & $\ldots$ & $\ldots$ & $\ldots$ & $\ldots$ \\
\hline DDO 053 & $214 \pm 033 \mathrm{E}-3$ & $207 \pm 032 \mathrm{E}-3$ & $660 \pm 160 \mathrm{E}-3$ & $820 \pm 200 \mathrm{E}-3$ & $550 \pm 180 \mathrm{E}-3$ & $800 \pm 260 \mathrm{E}-3$ & $271 \pm 014 \mathrm{E}-3$ & $507 \pm 017 \mathrm{E}-3$ & $602 \pm 020 \mathrm{E}-3$ & $624 \pm 021 \mathrm{E}-3$ \\
\hline NGC 2798 & $965 \pm 133 \mathrm{E}-4$ & $201 \pm 027 \mathrm{E}-3$ & $298 \pm 027 \mathrm{E}-2$ & $545 \pm 033 \mathrm{E}-2$ & $645 \pm 033 \mathrm{E}-2$ & $110 \pm 010 \mathrm{E}-1$ & $839 \pm 028 \mathrm{E}-3$ & $310 \pm 007 \mathrm{E}-2$ & $598 \pm 012 \mathrm{E}-2$ & $825 \pm 017 \mathrm{E}-2$ \\
\hline NGC 2841 & $116 \pm 016 \mathrm{E}-2$ & $183 \pm 025 \mathrm{E}-2$ & $435 \pm 020 \mathrm{E}-1$ & $765 \pm 027 \mathrm{E}-1$ & $101 \pm 007 \mathrm{E}+0$ & $176 \pm 009 \mathrm{E}+0$ & $111 \pm 002 \mathrm{E}-1$ & $459 \pm 009 \mathrm{E}-1$ & $941 \pm 018 \mathrm{E}-1$ & $140 \pm 002 \mathrm{E}+0$ \\
\hline NGC 2915 & $218 \pm 030 \mathrm{E}-3$ & $216 \pm 030 \mathrm{E}-3$ & $214 \pm 021 \mathrm{E}-2$ & $308 \pm 011 \mathrm{E}-2$ & $508 \pm 018 \mathrm{E}-2$ & $500 \pm 050 \mathrm{E}-2$ & $\ldots$ & $\ldots$ & $\ldots$ & $\ldots$ \\
\hline HoI & $369 \pm 058 \mathrm{E}-3$ & $401 \pm 061 \mathrm{E}-3$ & $116 \pm 029 \mathrm{E}-2$ & $153 \pm 038 \mathrm{E}-2$ & $147 \pm 043 \mathrm{E}-2$ & $211 \pm 053 \mathrm{E}-2$ & $\ldots$ & $\ldots$ & $\ldots$ & $\ldots$ \\
\hline NGC 2976 & $125 \pm 019 \mathrm{E}-2$ & $173 \pm 026 \mathrm{E}-2$ & $220 \pm 014 \mathrm{E}-1$ & $357 \pm 011 \mathrm{E}-1$ & $464 \pm 029 \mathrm{E}-1$ & $676 \pm 045 \mathrm{E}-1$ & $753 \pm 016 \mathrm{E}-2$ & $225 \pm 004 \mathrm{E}-1$ & $400 \pm 008 \mathrm{E}-1$ & $539 \pm 010 \mathrm{E}-1$ \\
\hline NGC 3049 & $228 \pm 034 \mathrm{E}-3$ & $339 \pm 046 \mathrm{E}-3$ & $246 \pm 020 \mathrm{E}-2$ & $359 \pm 018 \mathrm{E}-2$ & $397 \pm 028 \mathrm{E}-2$ & $532 \pm 051 \mathrm{E}-2$ & $923 \pm 027 \mathrm{E}-3$ & $234 \pm 005 \mathrm{E}-2$ & $390 \pm 008 \mathrm{E}-2$ & $499 \pm 010 \mathrm{E}-2$ \\
\hline NGC 3031 & $100 \pm 015 \mathrm{E}-1$ & $133 \pm 020 \mathrm{E}-1$ & $297 \pm 029 \mathrm{E}+0$ & $610 \pm 061 \mathrm{E}+0$ & $\ldots$ & $141 \pm 003 \mathrm{E}+1$ & $884 \pm 017 \mathrm{E}-1$ & $362 \pm 007 \mathrm{E}+0$ & $778 \pm 015 \mathrm{E}+0$ & $116 \pm 002 \mathrm{E}+1$ \\
\hline NGC 3034 & $101 \pm 015 \mathrm{E}-2$ & $288 \pm 043 \mathrm{E}-2$ & $111 \pm 018 \mathrm{E}+0$ & $164 \pm 016 \mathrm{E}+0$ & $243 \pm 033 \mathrm{E}+0$ & $376 \pm 045 \mathrm{E}+0$ & $323 \pm 006 \mathrm{E}-1$ & $105 \pm 002 \mathrm{E}+0$ & $231 \pm 004 \mathrm{E}+0$ & $345 \pm 006 \mathrm{E}+0$ \\
\hline HoIX & $226 \pm 031 \mathrm{E}-3$ & $280 \pm 038 \mathrm{E}-3$ & $570 \pm 090 \mathrm{E}-3$ & $550 \pm 110 \mathrm{E}-3$ & $590 \pm 120 \mathrm{E}-3$ & $900 \pm 300 \mathrm{E}-3$ & $375 \pm 019 \mathrm{E}-3$ & $635 \pm 020 \mathrm{E}-3$ & $807 \pm 024 \mathrm{E}-3$ & $930 \pm 028 \mathrm{E}-3$ \\
\hline NGC 3077 & $\ldots$ & $\ldots$ & $183 \pm 005 \mathrm{E}-1$ & $\ldots$ & $539 \pm 014 \mathrm{E}-1$ & $\ldots$ & $694 \pm 015 \mathrm{E}-2$ & $264 \pm 005 \mathrm{E}-1$ & $496 \pm 010 \mathrm{E}-1$ & $688 \pm 013 \mathrm{E}-1$ \\
\hline M81dwB & $469 \pm 073 \mathrm{E}-4$ & $586 \pm 090 \mathrm{E}-4$ & $480 \pm 160 \mathrm{E}-3$ & $650 \pm 210 \mathrm{E}-3$ & $800 \pm 270 \mathrm{E}-3$ & $830 \pm 230 \mathrm{E}-3$ & $175 \pm 011 \mathrm{E}-3$ & $447 \pm 016 \mathrm{E}-3$ & $682 \pm 021 \mathrm{E}-3$ & $842 \pm 025 \mathrm{E}-3$ \\
\hline NGC 3190 & $334 \pm 046 \mathrm{E}-4$ & $149 \pm 020 \mathrm{E}-3$ & $997 \pm 040 \mathrm{E}-2$ & $180 \pm 005 \mathrm{E}-1$ & $253 \pm 008 \mathrm{E}-1$ & $400 \pm 015 \mathrm{E}-1$ & $216 \pm 005 \mathrm{E}-2$ & $102 \pm 002 \mathrm{E}-1$ & $211 \pm 004 \mathrm{E}-1$ & $317 \pm 006 \mathrm{E}-1$ \\
\hline NGC 3184 & $373 \pm 051 \mathrm{E}-2$ & $492 \pm 068 \mathrm{E}-2$ & $336 \pm 029 \mathrm{E}-1$ & $478 \pm 020 \mathrm{E}-1$ & $566 \pm 039 \mathrm{E}-1$ & $740 \pm 041 \mathrm{E}-1$ & $106 \pm 002 \mathrm{E}-1$ & $316 \pm 006 \mathrm{E}-1$ & $509 \pm 010 \mathrm{E}-1$ & $667 \pm 013 \mathrm{E}-1$ \\
\hline NGC 3198 & $215 \pm 029 \mathrm{E}-2$ & $259 \pm 035 \mathrm{E}-2$ & $200 \pm 008 \mathrm{E}-1$ & $251 \pm 013 \mathrm{E}-1$ & $296 \pm 024 \mathrm{E}-1$ & $398 \pm 033 \mathrm{E}-1$ & $628 \pm 013 \mathrm{E}-2$ & $171 \pm 003 \mathrm{E}-1$ & $270 \pm 005 \mathrm{E}-1$ & $350 \pm 007 \mathrm{E}-1$ \\
\hline IC2574 & $390 \pm 061 \mathrm{E}-2$ & $405 \pm 061 \mathrm{E}-2$ & $140 \pm 019 \mathrm{E}-1$ & $184 \pm 025 \mathrm{E}-1$ & $182 \pm 029 \mathrm{E}-1$ & $264 \pm 035 \mathrm{E}-1$ & $\ldots$ & $\ldots$ & $\ldots$ & $\ldots$ \\
\hline NGC 3265 & $474 \pm 065 \mathrm{E}-4$ & $807 \pm 111 \mathrm{E}-4$ & $112 \pm 014 \mathrm{E}-2$ & $170 \pm 008 \mathrm{E}-2$ & $218 \pm 020 \mathrm{E}-2$ & $352 \pm 051 \mathrm{E}-2$ & $365 \pm 016 \mathrm{E}-3$ & $113 \pm 003 E-2$ & $209 \pm 00$ & $289 \pm 006 \mathrm{E}-2$ \\
\hline Mrk 33 & $378 \pm 052 \mathrm{E}-3$ & $475 \pm 065 \mathrm{E}-3$ & $196 \pm 014 \mathrm{E}-2$ & $283 \pm 055 \mathrm{E}-2$ & $336 \pm 073 \mathrm{E}-2$ & $392 \pm 078 \mathrm{E}-2$ & $937 \pm 027 \mathrm{E}-3$ & $197 \pm 004 \mathrm{E}-2$ & $299 \pm 006 \mathrm{E}-2$ & $342 \pm 007 \mathrm{E}-2$ \\
\hline NGC 3351 & $147 \pm 023 \mathrm{E}-2$ & $242 \pm 036 \mathrm{E}-2$ & $302 \pm 037 \mathrm{E}-1$ & $533 \pm 039 \mathrm{E}-1$ & $700 \pm 050 \mathrm{E}-1$ & $107 \pm 006 \mathrm{E}+0$ & $876 \pm 018 \mathrm{E}-2$ & $328 \pm 006 \mathrm{E}-1$ & $620 \pm 012 \mathrm{E}-1$ & $878 \pm 017 \mathrm{E}-1$ \\
\hline NGC 3521 & $144 \pm 023 \mathrm{E}-2$ & $296 \pm 045 \mathrm{E}-2$ & $629 \pm 024 \mathrm{E}-1$ & $107 \pm 002 \mathrm{E}+0$ & $147 \pm 002 \mathrm{E}+0$ & $225 \pm 006 \mathrm{E}+0$ & $170 \pm 003 \mathrm{E}-1$ & $664 \pm 013 \mathrm{E}-1$ & $129 \pm 002 \mathrm{E}+0$ & $180 \pm 003 \mathrm{E}+0$ \\
\hline NGC 3621 & $427 \pm 062 \mathrm{E}-2$ & $608 \pm 086 \mathrm{E}-2$ & $428 \pm 015 \mathrm{E}-1$ & $651 \pm 024 \mathrm{E}-1$ & $780 \pm 028 \mathrm{E}-1$ & $104 \pm 003 \mathrm{E}+0$ & $\ldots$ & $\ldots$ & $\ldots$ & $\ldots$ \\
\hline NGC 3627 & $246 \pm 038 \mathrm{E}-2$ & $452 \pm 069 \mathrm{E}-2$ & $742 \pm 042 \mathrm{E}-1$ & $115 \pm 002 \mathrm{E}+0$ & $141 \pm 012 \mathrm{E}+0$ & $215 \pm 011 \mathrm{E}+0$ & $214 \pm 004 \mathrm{E}-1$ & $760 \pm 015 \mathrm{E}-1$ & $136 \pm 002 \mathrm{E}+0$ & $190 \pm 003 \mathrm{E}+0$ \\
\hline NGC 3773 & $345 \pm 047 \mathrm{E}-3$ & $454 \pm 062 \mathrm{E}-3$ & $166 \pm 009 \mathrm{E}-2$ & $226 \pm 008 \mathrm{E}-2$ & $259 \pm 021 \mathrm{E}-2$ & $327 \pm 026 \mathrm{E}-2$ & $754 \pm 023 \mathrm{E}-3$ & $163 \pm 004 \mathrm{E}-2$ & $253 \pm 005 \mathrm{E}-2$ & $307 \pm 007 \mathrm{E}-2$ \\
\hline
\end{tabular}


Table 2

(Continued)

\begin{tabular}{|c|c|c|c|c|c|c|c|c|c|c|}
\hline Filter & $\begin{array}{c}\text { GALEX } \\
\text { FUV }\end{array}$ & $\begin{array}{c}\text { GALEX } \\
\text { NUV }\end{array}$ & $B$ & V & $R_{\mathrm{C}}$ & $I_{\mathrm{C}}$ & $\begin{array}{c}\text { SDSS } \\
u\end{array}$ & $\begin{array}{c}\text { SDSS } \\
\quad g\end{array}$ & $\begin{array}{c}\text { SDSS } \\
r\end{array}$ & $\begin{array}{c}\text { SDSS } \\
i\end{array}$ \\
\hline $\bar{\lambda}(\mu \mathrm{m})$ & 0.154 & 0.231 & 0.441 & 0.551 & 0.659 & 0.806 & 0.356 & 0.482 & 0.626 & 0.767 \\
\hline$\Delta \lambda(\mu \mathrm{m})$ & 0.025 & 0.073 & 0.096 & 0.089 & 0.159 & 0.150 & 0.056 & 0.127 & 0.133 & 0.135 \\
\hline$A_{\lambda} / A_{V}$ & 2.586 & 2.994 & 1.310 & 1.0 & 0.788 & 0.577 & 1.642 & 1.219 & 0.849 & 0.641 \\
\hline NGC 3938 & $\ldots$ & $311 \pm 043 \mathrm{E}-2$ & $218 \pm 008 \mathrm{E}-1$ & $308 \pm 005 \mathrm{E}-1$ & $347 \pm 019 \mathrm{E}-1$ & $486 \pm 017 \mathrm{E}-1$ & $724 \pm 015 \mathrm{E}-2$ & $203 \pm 004 \mathrm{E}-1$ & $314 \pm 006 \mathrm{E}-1$ & $407 \pm 008 \mathrm{E}-1$ \\
\hline NGC 4125 & $\ldots$ & $193 \pm 028 \mathrm{E}-3$ & $202 \pm 003 \mathrm{E}-1$ & $369 \pm 007 \mathrm{E}-1$ & $504 \pm 011 \mathrm{E}-1$ & $764 \pm 048 \mathrm{E}-1$ & $391 \pm 008 \mathrm{E}-2$ & $204 \pm 004 \mathrm{E}-1$ & $419 \pm 008 \mathrm{E}-1$ & $619 \pm 012 \mathrm{E}-1$ \\
\hline NGC 4236 & $625 \pm 098 \mathrm{E}-2$ & $744 \pm 113 \mathrm{E}-2$ & $343 \pm 029 \mathrm{E}-1$ & $395 \pm 031 \mathrm{E}-1$ & $479 \pm 038 \mathrm{E}-1$ & $560 \pm 054 \mathrm{E}-1$ & $\ldots$ & $\ldots$ & $\ldots$ & $\ldots$ \\
\hline NGC 4254 & $259 \pm 038 \mathrm{E}-2$ & $464 \pm 064 \mathrm{E}-2$ & $344 \pm 021 \mathrm{E}-1$ & $486 \pm 014 \mathrm{E}-1$ & $614 \pm 022 \mathrm{E}-1$ & $765 \pm 026 \mathrm{E}-1$ & $128 \pm 002 \mathrm{E}-1$ & $339 \pm 006 \mathrm{E}-1$ & $543 \pm 010 \mathrm{E}-1$ & $697 \pm 014 \mathrm{E}-1$ \\
\hline NGC 4321 & $262 \pm 039 \mathrm{E}-2$ & $445 \pm 061 \mathrm{E}-2$ & $442 \pm 020 \mathrm{E}-1$ & $670 \pm 017 \mathrm{E}-1$ & $889 \pm 041 \mathrm{E}-1$ & $132 \pm 007 \mathrm{E}+0$ & $152 \pm 003 \mathrm{E}-1$ & $442 \pm 008 \mathrm{E}-1$ & $755 \pm 015 \mathrm{E}-1$ & $103 \pm 002 \mathrm{E}+0$ \\
\hline NGC 4450 & $\ldots$ & $438 \pm 060 \mathrm{E}-3$ & $201 \pm 010 \mathrm{E}-1$ & $368 \pm 010 \mathrm{E}-1$ & $505 \pm 017 \mathrm{E}-1$ & $768 \pm 032 \mathrm{E}-1$ & $513 \pm 011 \mathrm{E}-2$ & $213 \pm 004 \mathrm{E}-1$ & $432 \pm 008 \mathrm{E}-1$ & $629 \pm 012 \mathrm{E}-1$ \\
\hline NGC 4536 & $148 \pm 020 \mathrm{E}-2$ & $191 \pm 026 \mathrm{E}-2$ & $185 \pm 012 \mathrm{E}-1$ & $273 \pm 023 \mathrm{E}-1$ & $343 \pm 035 \mathrm{E}-1$ & $520 \pm 100 \mathrm{E}-1$ & $561 \pm 012 \mathrm{E}-2$ & $172 \pm 003 \mathrm{E}-1$ & $292 \pm 005 \mathrm{E}-1$ & $393 \pm 007 \mathrm{E}-1$ \\
\hline NGC 4552 & $140 \pm 019 \mathrm{E}-3$ & $344 \pm 047 \mathrm{E}-3$ & $230 \pm 009 \mathrm{E}-1$ & $425 \pm 010 \mathrm{E}-1$ & $603 \pm 016 \mathrm{E}-1$ & $942 \pm 051 \mathrm{E}-1$ & $440 \pm 009 \mathrm{E}-2$ & $239 \pm 004 \mathrm{E}-1$ & $501 \pm 010 \mathrm{E}-1$ & $749 \pm 015 \mathrm{E}-1$ \\
\hline NGC 4559 & $473 \pm 065 \mathrm{E}-2$ & $568 \pm 078 \mathrm{E}-2$ & $305 \pm 019 \mathrm{E}-1$ & $396 \pm 011 \mathrm{E}-1$ & $452 \pm 033 \mathrm{E}-1$ & $570 \pm 039 \mathrm{E}-1$ & $108 \pm 002 \mathrm{E}-1$ & $277 \pm 005 \mathrm{E}-1$ & $402 \pm 008 \mathrm{E}-1$ & $502 \pm 010 \mathrm{E}-1$ \\
\hline NGC 4569 & $427 \pm 059 \mathrm{E}-3$ & $139 \pm 019 \mathrm{E}-2$ & $335 \pm 033 \mathrm{E}-1$ & $555 \pm 055 \mathrm{E}-1$ & $\cdots$ & $\cdots$ & $909 \pm 019 \mathrm{E}-2$ & $361 \pm 007 \mathrm{E}-1$ & $624 \pm 012 \mathrm{E}-1$ & $900 \pm 018 \mathrm{E}-1$ \\
\hline NGC 4579 & $434 \pm 060 \mathrm{E}-3$ & $897 \pm 124 \mathrm{E}-3$ & $293 \pm 012 \mathrm{E}-1$ & $535 \pm 020 \mathrm{E}-1$ & $694 \pm 043 \mathrm{E}-1$ & $105 \pm 006 \mathrm{E}+0$ & $661 \pm 014 \mathrm{E}-2$ & $299 \pm 006 \mathrm{E}-1$ & $602 \pm 012 \mathrm{E}-1$ & $883 \pm 017 \mathrm{E}-1$ \\
\hline NGC 4594 & $376 \pm 059 \mathrm{E}-3$ & $111 \pm 017 \mathrm{E}-2$ & $103 \pm 006 \mathrm{E}+0$ & $242 \pm 032 \mathrm{E}+0$ & $292 \pm 009 \mathrm{E}+0$ & $453 \pm 028 \mathrm{E}+0$ & $242 \pm 005 \mathrm{E}-1$ & $123 \pm 002 \mathrm{E}+0$ & $265 \pm 005 \mathrm{E}+0$ & $401 \pm 008 \mathrm{E}+0$ \\
\hline NGC 4625 & $549 \pm 087 \mathrm{E}-3$ & $698 \pm 107 \mathrm{E}-3$ & $364 \pm 028 \mathrm{E}-2$ & $527 \pm 047 \mathrm{E}-2$ & $617 \pm 086 \mathrm{E}-2$ & $805 \pm 058 \mathrm{E}-2$ & $126 \pm 003 \mathrm{E}-2$ & $341 \pm 007 \mathrm{E}-2$ & $534 \pm 011 \mathrm{E}-2$ & $686 \pm 014 \mathrm{E}-2$ \\
\hline NGC 4631 & $963 \pm 151 \mathrm{E}-2$ & $118 \pm 018 \mathrm{E}-1$ & $616 \pm 048 \mathrm{E}-1$ & $812 \pm 030 \mathrm{E}-1$ & $944 \pm 048 \mathrm{E}-1$ & $119 \pm 005 \mathrm{E}+0$ & $249 \pm 005 \mathrm{E}-1$ & $579 \pm 011 \mathrm{E}-1$ & $854 \pm 017 \mathrm{E}-1$ & $108 \pm 002 \mathrm{E}+0$ \\
\hline NGC 4725 & $202 \pm 028 \mathrm{E}-2$ & $271 \pm 037 \mathrm{E}-2$ & $466 \pm 024 \mathrm{E}-1$ & $825 \pm 022 \mathrm{E}-1$ & $104 \pm 003 \mathrm{E}+0$ & $163 \pm 007 \mathrm{E}+0$ & $123 \pm 002 \mathrm{E}-1$ & $478 \pm 009 \mathrm{E}-1$ & $910 \pm 018 \mathrm{E}-1$ & $131 \pm 002 \mathrm{E}+0$ \\
\hline NGC 4736 & $625 \pm 098 \mathrm{E}-2$ & $809 \pm 123 \mathrm{E}-2$ & $141 \pm 009 \mathrm{E}+0$ & $215 \pm 006 \mathrm{E}+0$ & $292 \pm 010 \mathrm{E}+0$ & $431 \pm 024 \mathrm{E}+0$ & $415 \pm 008 \mathrm{E}-1$ & $162 \pm 003 \mathrm{E}+0$ & $300 \pm 006 \mathrm{E}+0$ & $421 \pm 008 \mathrm{E}+0$ \\
\hline DDO 154 & $413 \pm 064 \mathrm{E}-3$ & $401 \pm 062 \mathrm{E}-3$ & $105 \pm 029 \mathrm{E}-2$ & $113 \pm 035 \mathrm{E}-2$ & $121 \pm 030 \mathrm{E}-2$ & $127 \pm 032 \mathrm{E}-2$ & $450 \pm 017 \mathrm{E}-3$ & $892 \pm 026 \mathrm{E}-3$ & $102 \pm 002 \mathrm{E}-2$ & $102 \pm 002 \mathrm{E}-2$ \\
\hline NGC 4826 & $100 \pm 015 \mathrm{E}-2$ & $258 \pm 039 \mathrm{E}-2$ & $957 \pm 078 \mathrm{E}-1$ & $149 \pm 008 \mathrm{E}+0$ & $190 \pm 017 \mathrm{E}+0$ & $303 \pm 017 \mathrm{E}+0$ & $228 \pm 004 \mathrm{E}-1$ & $966 \pm 019 \mathrm{E}-1$ & $184 \pm 003 \mathrm{E}+0$ & $268 \pm 005 \mathrm{E}+0$ \\
\hline DDO 165 & $483 \pm 075 \mathrm{E}-3$ & $660 \pm 100 \mathrm{E}-3$ & $285 \pm 071 \mathrm{E}-2$ & $277 \pm 028 \mathrm{E}-2$ & $254 \pm 055 \mathrm{E}-2$ & $339 \pm 125 \mathrm{E}-2$ & $929 \pm 028 \mathrm{E}-3$ & $220 \pm 005 \mathrm{E}-2$ & $255 \pm 006 \mathrm{E}-2$ & $284 \pm 006 \mathrm{E}-2$ \\
\hline NGC 5033 & $181 \pm 025 \mathrm{E}-2$ & $235 \pm 032 \mathrm{E}-2$ & $241 \pm 012 \mathrm{E}-1$ & $392 \pm 021 \mathrm{E}-1$ & $\ldots$ & $636 \pm 041 \mathrm{E}-1$ & $734 \pm 015 \mathrm{E}-2$ & $235 \pm 004 \mathrm{E}-1$ & $422 \pm 008 \mathrm{E}-1$ & $581 \pm 011 \mathrm{E}-1$ \\
\hline NGC 5055 & $369 \pm 058 \mathrm{E}-2$ & $586 \pm 090 \mathrm{E}-2$ & $804 \pm 080 \mathrm{E}-1$ & $133 \pm 013 \mathrm{E}+0$ & $181 \pm 005 \mathrm{E}+0$ & $274 \pm 007 \mathrm{E}+0$ & $280 \pm 005 \mathrm{E}-1$ & $934 \pm 018 \mathrm{E}-1$ & $171 \pm 003 \mathrm{E}+0$ & $245 \pm 004 \mathrm{E}+0$ \\
\hline NGC 5194 & $107 \pm 016 \mathrm{E}-1$ & $165 \pm 025 \mathrm{E}-1$ & $133 \pm 015 \mathrm{E}+0$ & $182 \pm 012 \mathrm{E}+0$ & $215 \pm 020 \mathrm{E}+0$ & $323 \pm 029 \mathrm{E}+0$ & $568 \pm 011 \mathrm{E}-1$ & $149 \pm 003 \mathrm{E}+0$ & $249 \pm 005 \mathrm{E}+0$ & $335 \pm 006 \mathrm{E}+0$ \\
\hline NGC 5195 & $155 \pm 025 \mathrm{E}-3$ & $420 \pm 066 \mathrm{E}-3$ & $373 \pm 042 \mathrm{E}-1$ & $612 \pm 041 \mathrm{E}-1$ & $806 \pm 076 \mathrm{E}-1$ & $150 \pm 013 \mathrm{E}+0$ & $675 \pm 014 \mathrm{E}-2$ & $261 \pm 005 \mathrm{E}-1$ & $562 \pm 011 \mathrm{E}-1$ & $852 \pm 017 \mathrm{E}-1$ \\
\hline NGC 5398 & $469 \pm 064 \mathrm{E}-3$ & $699 \pm 096 \mathrm{E}-3$ & $481 \pm 048 \mathrm{E}-2$ & $506 \pm 050 \mathrm{E}-2$ & $403 \pm 040 \mathrm{E}-2$ & $520 \pm 052 \mathrm{E}-2$ & $\cdots$ & $\cdots$ & $\cdots$ & $\cdots$ \\
\hline NGC 5457 & $346 \pm 054 \mathrm{E}-1$ & $387 \pm 059 \mathrm{E}-1$ & $202 \pm 017 \mathrm{E}+0$ & $261 \pm 024 \mathrm{E}+0$ & $285 \pm 007 \mathrm{E}+0$ & $431 \pm 011 \mathrm{E}+0$ & $755 \pm 015 \mathrm{E}-1$ & $189 \pm 003 \mathrm{E}+0$ & $275 \pm 005 \mathrm{E}+0$ & $352 \pm 007 \mathrm{E}+0$ \\
\hline NGC 5408 & $\ldots$ & $\ldots$ & $562 \pm 056 \mathrm{E}-2$ & $804 \pm 080 \mathrm{E}-2$ & $\ldots$ & $\ldots$ & $\ldots$ & $\ldots$ & $\cdots$ & $\ldots$ \\
\hline NGC 5474 & $231 \pm 036 \mathrm{E}-2$ & $251 \pm 038 \mathrm{E}-2$ & $124 \pm 007 \mathrm{E}-1$ & $165 \pm 006 \mathrm{E}-1$ & $180 \pm 012 \mathrm{E}-1$ & $215 \pm 019 \mathrm{E}-1$ & $488 \pm 011 \mathrm{E}-2$ & $116 \pm 002 \mathrm{E}-1$ & $166 \pm 003 \mathrm{E}-1$ & $199 \pm 004 \mathrm{E}-1$ \\
\hline NGC 5713 & $387 \pm 053 \mathrm{E}-3$ & $750 \pm 103 E-3$ & $920 \pm 062 \mathrm{E}-2$ & $134 \pm 006 \mathrm{E}-1$ & $174 \pm 006 \mathrm{E}-1$ & $237 \pm 025 \mathrm{E}-1$ & $285 \pm 006 \mathrm{E}-2$ & $884 \pm 018 \mathrm{E}-2$ & $151 \pm 003 \mathrm{E}-1$ & $197 \pm 004 \mathrm{E}-1$ \\
\hline NGC 5866 & $584 \pm 081 \mathrm{E}-4$ & $375 \pm 051 \mathrm{E}-3$ & $235 \pm 010 \mathrm{E}-1$ & $429 \pm 015 \mathrm{E}-1$ & $542 \pm 037 \mathrm{E}-1$ & $849 \pm 049 \mathrm{E}-1$ & $556 \pm 012 \mathrm{E}-2$ & $255 \pm 005 \mathrm{E}-1$ & $493 \pm 009 \mathrm{E}-1$ & $731 \pm 014 \mathrm{E}-1$ \\
\hline IC4710 & $118 \pm 016 \mathrm{E}-2$ & $157 \pm 021 \mathrm{E}-2$ & $566 \pm 056 \mathrm{E}-2$ & $797 \pm 079 \mathrm{E}-2$ & $704 \pm 070 \mathrm{E}-2$ & $\ldots$ & $\ldots$ & $\ldots$ & $\ldots$ & $\ldots$ \\
\hline NGC 6822 & $573 \pm 079 \mathrm{E}-2$ & $739 \pm 103 E-2$ & $658 \pm 034 \mathrm{E}-1$ & $973 \pm 037 \mathrm{E}-1$ & $121 \pm 004 \mathrm{E}+0$ & $977 \pm 085 \mathrm{E}-1$ & $\ldots$ & $\ldots$ & $\ldots$ & $\ldots$ \\
\hline NGC 6946 & $183 \pm 025 \mathrm{E}-2$ & $335 \pm 046 \mathrm{E}-2$ & $610 \pm 060 \mathrm{E}-1$ & $127 \pm 005 \mathrm{E}+0$ & $\ldots$ & $349 \pm 034 \mathrm{E}+0$ & $\ldots$ & $\ldots$ & $\ldots$ & $\ldots$ \\
\hline NGC 7331 & $802 \pm 111 \mathrm{E}-3$ & $151 \pm 020 \mathrm{E}-2$ & $452 \pm 026 \mathrm{E}-1$ & $715 \pm 013 \mathrm{E}-1$ & $101 \pm 002 \mathrm{E}+0$ & $168 \pm 007 \mathrm{E}+0$ & $905 \pm 019 \mathrm{E}-2$ & $386 \pm 007 \mathrm{E}-1$ & $814 \pm 016 \mathrm{E}-1$ & $124 \pm 002 \mathrm{E}+0$ \\
\hline NGC 7552 & $697 \pm 096 \mathrm{E}-3$ & $136 \pm 019 \mathrm{E}-2$ & $127 \pm 004 \mathrm{E}-1$ & $216 \pm 007 \mathrm{E}-1$ & $226 \pm 022 \mathrm{E}-1$ & $214 \pm 021 \mathrm{E}-1$ & $\cdots$ & $\cdots$ & $\cdots$ & $\ldots$ \\
\hline \multirow[t]{2}{*}{ NGC 7793} & $111 \pm 017 \mathrm{E}-1$ & $129 \pm 019 \mathrm{E}-1$ & $550 \pm 055 \mathrm{E}-1$ & $766 \pm 076 \mathrm{E}-1$ & $745 \pm 074 \mathrm{E}-1$ & $661 \pm 066 \mathrm{E}-1$ & $\cdots$ & $\cdots$ & $\cdots$ & $\cdots$ \\
\hline & SDSS & 2MASS & 2MASS & 2MASS & Spitzer & Spitzer & Spitzer & Spitzer & WISE & Spitzer \\
\hline Filter & $z$ & $J$ & $H$ & $K_{\mathrm{s}}$ & IRAC & IRAC & IRAC & IRAC & W3 & MIPS \\
\hline $\bar{\lambda}(\mu \mathrm{m})$ & 0.910 & 1.24 & 1.65 & 2.17 & 3.56 & 4.51 & 5.76 & 7.96 & 12.8 & 23.8 \\
\hline$\Delta \lambda(\mu \mathrm{m})$ & 0.131 & 0.163 & 0.252 & 0.264 & 0.687 & 0.872 & 1.25 & 2.55 & 5.51 & 5.32 \\
\hline$A_{\lambda} / A_{V}$ & 0.488 & 0.296 & 0.187 & 0.116 & 0.0451 & 0.0288 & 0.0193 & 0.0296 & 0.0355 & 0.0193 \\
\hline NGC 0024 & $\cdots$ & $228 \pm 012 \mathrm{E}-1$ & $246 \pm 013 \mathrm{E}-1$ & $188 \pm 012 \mathrm{E}-1$ & $101 \pm 013 \mathrm{E}-1$ & $682 \pm 101 \mathrm{E}-2$ & $821 \pm 116 \mathrm{E}-2$ & $117 \pm 016 \mathrm{E}-1$ & $924 \pm 064 \mathrm{E}-2$ & $120 \pm 004 \mathrm{E}-1$ \\
\hline NGC 0337 & $136 \pm 002 \mathrm{E}-1$ & $178 \pm 009 \mathrm{E}-1$ & $190 \pm 009 \mathrm{E}-1$ & $164 \pm 008 \mathrm{E}-1$ & $958 \pm 126 \mathrm{E}-2$ & $659 \pm 090 \mathrm{E}-2$ & $141 \pm 018 \mathrm{E}-1$ & $376 \pm 047 \mathrm{E}-1$ & $291 \pm 020 \mathrm{E}-1$ & $673 \pm 028 \mathrm{E}-1$ \\
\hline NGC 0584 & $562 \pm 011 \mathrm{E}-1$ & $880 \pm 044 \mathrm{E}-1$ & $109 \pm 005 \mathrm{E}+0$ & $860 \pm 043 \mathrm{E}-1$ & $365 \pm 049 \mathrm{E}-1$ & $219 \pm 029 \mathrm{E}-1$ & $174 \pm 022 \mathrm{E}-1$ & $114 \pm 014 \mathrm{E}-1$ & $700 \pm 049 \mathrm{E}-2$ & $480 \pm 020 \mathrm{E}-2$ \\
\hline
\end{tabular}


Table 2

(Continued)

\begin{tabular}{|c|c|c|c|c|c|c|c|c|c|c|}
\hline Filter & $\begin{array}{c}\text { SDSS } \\
z\end{array}$ & $\underset{J}{2 \text { MASS }}$ & $\begin{array}{c}\text { 2MASS } \\
H\end{array}$ & $\begin{array}{c}\text { 2MASS } \\
K_{\mathrm{s}}\end{array}$ & $\begin{array}{c}\text { Spitzer } \\
\text { IRAC }\end{array}$ & $\begin{array}{c}\text { Spitzer } \\
\text { IRAC }\end{array}$ & $\begin{array}{c}\text { Spitzer } \\
\text { IRAC }\end{array}$ & $\begin{array}{c}\text { Spitzer } \\
\text { IRAC }\end{array}$ & $\begin{array}{c}\text { WISE } \\
\text { W3 }\end{array}$ & $\begin{array}{c}\text { Spitzer } \\
\text { MIPS }\end{array}$ \\
\hline $\bar{\lambda}(\mu \mathrm{m})$ & 0.910 & 1.24 & 1.65 & 2.17 & 3.56 & 4.51 & 5.76 & 7.96 & 12.8 & 23.8 \\
\hline$\Delta \lambda(\mu \mathrm{m})$ & 0.131 & 0.163 & 0.252 & 0.264 & 0.687 & 0.872 & 1.25 & 2.55 & 5.51 & 5.32 \\
\hline$A_{\lambda} / A_{V}$ & 0.488 & 0.296 & 0.187 & 0.116 & 0.0451 & 0.0288 & 0.0193 & 0.0296 & 0.0355 & 0.0193 \\
\hline NGC 0628 & $123 \pm 002 \mathrm{E}+0$ & $156 \pm 007 \mathrm{E}+0$ & $160 \pm 008 \mathrm{E}+0$ & $128 \pm 006 \mathrm{E}+0$ & $839 \pm 117 \mathrm{E}-1$ & $544 \pm 075 \mathrm{E}-1$ & $116 \pm 014 \mathrm{E}+0$ & $259 \pm 033 \mathrm{E}+0$ & $235 \pm 016 \mathrm{E}+0$ & $323 \pm 012 \mathrm{E}+0$ \\
\hline NGC 0855 & $766 \pm 017 \mathrm{E}-2$ & $53 \pm 051 \mathrm{E}-2$ & $940 \pm 064 \mathrm{E}-2$ & $778 \pm 066 \mathrm{E}-2$ & $28 \pm 060 \mathrm{E}-2$ & $280 \pm 039 \mathrm{E}-2$ & $359 \pm 030 \mathrm{E}-2$ & $474 \pm 057 \mathrm{E}-2$ & $354 \pm 024 \mathrm{E}-2$ & $51 \pm 0$ \\
\hline NGC 0925 & $\ldots$ & $561 \pm 028 \mathrm{E}-1$ & $621 \pm 031 \mathrm{E}-1$ & $500 \pm 026 \mathrm{E}-1$ & $307 \pm 041 \mathrm{E}-1$ & $210 \pm 028 \mathrm{E}-1$ & $349 \pm 044 \mathrm{E}-1$ & $606 \pm 075 \mathrm{E}-1$ & $545 \pm 038 \mathrm{E}-1$ & $943 \pm 040 \mathrm{E}-1$ \\
\hline NGC 1097 & $\ldots$ & $234 \pm 011 \mathrm{E}+0$ & $270 \pm 013 \mathrm{E}+0$ & $226 \pm 011 \mathrm{E}+0$ & $123 \pm 016 \mathrm{E}+0$ & $800 \pm 109 \mathrm{E}-1$ & $146 \pm 018 \mathrm{E}+0$ & $317 \pm 039 \mathrm{E}+0$ & $298 \pm 020 \mathrm{E}+0$ & $661 \pm 027 \mathrm{E}+0$ \\
\hline NGC 1266 & $\ldots$ & $112 \pm 005 \mathrm{E}-1$ & $120 \pm 006 \mathrm{E}-1$ & $116 \pm 006 \mathrm{E}-1$ & $538 \pm 073 \mathrm{E}-2$ & $414 \pm 054 \mathrm{E}-2$ & $542 \pm 072 \mathrm{E}-2$ & $878 \pm 111 \mathrm{E}-2$ & $111 \pm 007 \mathrm{E}-1$ & $872 \pm 036 \mathrm{E}-1$ \\
\hline NGC 1291 & $\ldots$ & $432 \pm 021 \mathrm{E}+0$ & $453 \pm 022 \mathrm{E}+0$ & $396 \pm 019 \mathrm{E}+0$ & $207 \pm 028 \mathrm{E}+0$ & $127 \pm 017 \mathrm{E}+0$ & $906 \pm 121 \mathrm{E}-1$ & $639 \pm 079 \mathrm{E}-1$ & $592 \pm 041 \mathrm{E}-1$ & $480 \pm 019 \mathrm{E}-1$ \\
\hline NGC 1316 & $\ldots$ & $461 \pm 023 \mathrm{E}+0$ & $484 \pm 024 \mathrm{E}+0$ & $417 \pm 020 \mathrm{E}+0$ & $247 \pm 033 \mathrm{E}+0$ & $153 \pm 021 \mathrm{E}+0$ & $112 \pm 014 \mathrm{E}+0$ & $553 \pm 069 \mathrm{E}-1$ & $605 \pm 042 \mathrm{E}-1$ & $428 \pm 021 \mathrm{E}-1$ \\
\hline NGC 1377 & $\ldots$ & $986 \pm 051 \mathrm{E}-2$ & $113 \pm 006 \mathrm{E}-1$ & $936 \pm 053 \mathrm{E}-2$ & $567 \pm 075 \mathrm{E}-2$ & $850 \pm 116 \mathrm{E}-2$ & $267 \pm 035 \mathrm{E}-1$ & $419 \pm 052 \mathrm{E}-1$ & $397 \pm 027 \mathrm{E}-1$ & $182 \pm 007 \mathrm{E}+0$ \\
\hline NGC 1404 & $\ldots$ & $136 \pm 006 \mathrm{E}+0$ & $158 \pm 007 \mathrm{E}+0$ & $134 \pm 006 \mathrm{E}+0$ & $727 \pm 098 \mathrm{E}-1$ & $434 \pm 059 \mathrm{E}-1$ & $331 \pm 042 \mathrm{E}-1$ & $158 \pm 020 \mathrm{E}-1$ & $137 \pm 009 \mathrm{E}-1$ & $880 \pm 040 \mathrm{E}-2$ \\
\hline IC0342 & $\ldots$ & $952 \pm 047 \mathrm{E}+0$ & $106 \pm 005 \mathrm{E}+1$ & $107 \pm 005 \mathrm{E}+1$ & $125 \pm 016 \mathrm{E}+1$ & $701 \pm 096 \mathrm{E}+0$ & $113 \pm 014 \mathrm{E}+1$ & $251 \pm 031 \mathrm{E}+1$ & $233 \pm 016 \mathrm{E}+1$ & $362 \pm 014 \mathrm{E}+1$ \\
\hline NGC 1482 & $\ldots$ & $225 \pm 011 \mathrm{E}-1$ & $294 \pm 015 \mathrm{E}-1$ & $290 \pm 015 \mathrm{E}-1$ & $204 \pm 028 \mathrm{E}-1$ & $151 \pm 020 \mathrm{E}-1$ & $592 \pm 076 \mathrm{E}-1$ & $155 \pm 019 \mathrm{E}+0$ & $123 \pm 008 \mathrm{E}+0$ & $367 \pm 014 \mathrm{E}+0$ \\
\hline NGC 1512 & $\ldots$ & $4 \pm 043 \mathrm{E}-1$ & $851 \pm 048 \mathrm{E}-1$ & $727 \pm 046 \mathrm{E}-1$ & $38 \pm 052 \mathrm{E}-1$ & $294 \pm 033 \mathrm{E}-1$ & $260 \pm 034 \mathrm{E}-1$ & $456 \pm 054 \mathrm{E}-1$ & $448 \pm 031 \mathrm{E}-1$ & $486 \pm 019 \mathrm{E}-1$ \\
\hline NGC 1566 & $\ldots$ & $138 \pm 006 \mathrm{E}+0$ & $140 \pm 007 \mathrm{E}+0$ & $126 \pm 006 \mathrm{E}+0$ & $750 \pm 101 \mathrm{E}-1$ & $479 \pm 065 E-1$ & $907 \pm 115 \mathrm{E}-1$ & $211 \pm 026 \mathrm{E}+0$ & $188 \pm 013 \mathrm{E}+0$ & $282 \pm 012 \mathrm{E}+0$ \\
\hline NGC 1705 & $\ldots$ & $570 \pm 036 \mathrm{E}-2$ & $537 \pm 044 \mathrm{E}-2$ & $442 \pm 047 \mathrm{E}-2$ & $266 \pm 036 \mathrm{E}-2$ & $192 \pm 025 \mathrm{E}-2$ & $183 \pm 019 \mathrm{E}-2$ & $192 \pm 020 \mathrm{E}-2$ & $194 \pm 013 \mathrm{E}-2$ & $538 \pm 022 \mathrm{E}-2$ \\
\hline NGC 2146 & $\ldots$ & $892 \pm 044 \mathrm{E}-1$ & $117 \pm 005 \mathrm{E}+0$ & $107 \pm 005 \mathrm{E}+0$ & $835 \pm 113 \mathrm{E}-1$ & $629 \pm 086 \mathrm{E}-1$ & $250 \pm 032 \mathrm{E}+0$ & $676 \pm 084 \mathrm{E}+0$ & $495 \pm 034 \mathrm{E}+0$ & $123 \pm 004 \mathrm{E}+1$ \\
\hline NGC 2403 & $329 \pm 006 \mathrm{E}+0$ & $283 \pm 014 \mathrm{E}+0$ & $284 \pm 014 \mathrm{E}+0$ & $235 \pm 012 \mathrm{E}+0$ & $149 \pm 025 \mathrm{E}+0$ & $101 \pm 017 \mathrm{E}+0$ & $197 \pm 026 \mathrm{E}+0$ & $386 \pm 051 \mathrm{E}+0$ & $395 \pm 027 \mathrm{E}+0$ & $587 \pm 023 \mathrm{E}+0$ \\
\hline HoII & $\ldots$ & $275 \pm 016 \mathrm{E}-1$ & $294 \pm 019 \mathrm{E}-1$ & $226 \pm 018 \mathrm{E}-1$ & $771 \pm 098 \mathrm{E}-2$ & $644 \pm 078 \mathrm{E}-2$ & $399 \pm 047 \mathrm{E}-2$ & $440 \pm 048 \mathrm{E}-2$ & $417 \pm 030 \mathrm{E}-2$ & $177 \pm 007 \mathrm{E}-1$ \\
\hline M81dwA & $\ldots$ & $370 \pm 066 \mathrm{E}-3$ & $384 \pm 098 \mathrm{E}-3$ & $290 \pm 117 \mathrm{E}-3$ & $5 \pm 094 \mathrm{E}-3$ & $960 \pm 960 \mathrm{E}-4$ & $<376 \mathrm{E}-3$ & $<236 \mathrm{E}-3$ & $<630 \mathrm{E}-4$ & $<173 \mathrm{E}-2$ \\
\hline DDO 053 & $583 \pm 033 \mathrm{E}-3$ & $730 \pm 200 \mathrm{E}-3$ & $134 \pm 030 \mathrm{E}-2$ & $783 \pm 366 \mathrm{E}-3$ & $425 \pm 100 \mathrm{E}-3$ & $309 \pm 100 \mathrm{E}-3$ & $264 \pm 090 \mathrm{E}-3$ & $437 \pm 100 \mathrm{E}-3$ & $642 \pm 047 \mathrm{E}-3$ & $239 \pm 010 \mathrm{E}-2$ \\
\hline NGC 2798 & $106 \pm 002 \mathrm{E}-1$ & $160 \pm 008 \mathrm{E}-1$ & $184 \pm 009 \mathrm{E}-1$ & $172 \pm 009 \mathrm{E}-1$ & $114 \pm 015 \mathrm{E}-1$ & $810 \pm 114 \mathrm{E}-2$ & $65 \pm 034 \mathrm{E}-1$ & $632 \pm 078 \mathrm{E}-1$ & $7 \pm 041 \mathrm{E}-1$ & $261 \pm 010 \mathrm{E}+0$ \\
\hline NGC 2841 & $179 \pm 003 \mathrm{E}+0$ & $277 \pm 013 \mathrm{E}+0$ & $319 \pm 015 \mathrm{E}+0$ & $265 \pm 013 \mathrm{E}+0$ & $126 \pm 017 \mathrm{E}+0$ & $750 \pm 103 \mathrm{E}-1$ & $69 \pm 085 \mathrm{E}-1$ & $115 \pm 0$ & $00 \pm 007 \mathrm{E}+0$ & $908 \pm 037 \mathrm{E}-1$ \\
\hline NGC 2915 & $\ldots$ & $103 \pm 005 \mathrm{E}-1$ & $127 \pm 006 \mathrm{E}-1$ & $839 \pm 048 \mathrm{E}-2$ & $1 \pm 072 \mathrm{E}-2$ & $340 \pm 049 \mathrm{E}-2$ & $21 \pm 043 \mathrm{E}-2$ & $304 \pm 0$ & $80 \pm 012 \mathrm{E}-2$ & $620 \pm 026 \mathrm{E}-2$ \\
\hline HoI & $\ldots$ & $296 \pm 041 \mathrm{E}-2$ & $5 \pm 062 \mathrm{E}-2$ & $7 \pm 072 \mathrm{E}-2$ & $9 \pm 1$ & $595 \pm 1$ & -3 & $362=$ & -3 & $654 \pm$ \\
\hline NGC 2976 & $647 \pm 013 \mathrm{E}-1$ & $8 \pm 0$ & $8 \pm 0$ & $9 \pm 0$ & $405 \pm 0$ & $1 \pm$ & -1 & $101=$ & -1 & $139=$ \\
\hline NGC 3049 & $601 \pm 013 \mathrm{E}-2$ & $1 \pm 040 \mathrm{E}-2$ & $16 \mathrm{E}-2$ & $729 \pm$ & $2 \pm 05$ & $273 \pm$ & $1 \pm$ & $134 \pm$ & $112 \pm$ & $426 \pm$ \\
\hline NGC 3031 & $153 \pm 003 \mathrm{E}+1$ & $219 \pm 010 \mathrm{E}+1$ & $243 \pm 012 \mathrm{E}+1$ & $207 \pm 010 \mathrm{E}+1$ & $105 \pm 014 \mathrm{E}+1$ & $652 \pm 089 \mathrm{E}+0$ & $556 \pm 074 \mathrm{E}+0$ & $759 \pm 099 \mathrm{E}+0$ & $580 \pm 040 \mathrm{E}+0$ & $521 \pm 020 \mathrm{E}+0$ \\
\hline NGC 3034 & $461 \pm 009 \mathrm{E}+0$ & $11 \pm 040 \mathrm{E}+0$ & $992 \pm 049 \mathrm{E}+0$ & $960 \pm 048 \mathrm{E}+0$ & $712 \pm 223 \mathrm{E}+0$ & $567 \pm 178 \mathrm{E}+0$ & $234 \pm 072 \mathrm{E}+1$ & $613 \pm 189 \mathrm{E}+1$ & $713 \pm 050 \mathrm{E}+1$ & $322 \pm 096 \mathrm{E}+2^{\mathrm{a}}$ \\
\hline HoIX & $902 \pm 060 \mathrm{E}-3$ & $231 \pm 020 \mathrm{E}-2$ & $196 \pm 027 \mathrm{E}-2$ & $142 \pm 032 \mathrm{E}-2$ & $730 \pm 113 \mathrm{E}-3$ & $377 \pm 101 \mathrm{E}-3$ & $<130 \mathrm{E}-2$ & $<119 \mathrm{E}-2$ & $526 \pm 050 \mathrm{E}-3$ & $<362 \mathrm{E}-2$ \\
\hline NGC 3077 & $821 \pm 016 \mathrm{E}-1$ & $993 \pm 050 \mathrm{E}-1$ & $100 \pm 005 \mathrm{E}+0$ & $850 \pm 044 \mathrm{E}-1$ & $533 \pm 072 \mathrm{E}-1$ & $360 \pm 049 \mathrm{E}-1$ & $430 \pm 054 \mathrm{E}-1$ & $808 \pm 100 \mathrm{E}-1$ & $748 \pm 052 \mathrm{E}-1$ & $129 \pm 005 \mathrm{E}+0$ \\
\hline M81dwB & $997 \pm 037 \mathrm{E}-3$ & $111 \pm 014 \mathrm{E}-2$ & $130 \pm 022 \mathrm{E}-2$ & $132 \pm 026 \mathrm{E}-2$ & $534 \pm 100 \mathrm{E}-3$ & $360 \pm 100 \mathrm{E}-3$ & $321 \pm 090 \mathrm{E}-3$ & $307 \pm 080 \mathrm{E}-3$ & $373 \pm 124 \mathrm{E}-3$ & $332 \pm 034 \mathrm{E}-3$ \\
\hline NGC 3190 & $423 \pm 008 \mathrm{E}-1$ & $697 \pm 034 \mathrm{E}-1$ & $824 \pm 041 \mathrm{E}-1$ & $734 \pm 036 \mathrm{E}-1$ & $371 \pm 050 \mathrm{E}-1$ & $234 \pm 032 \mathrm{E}-1$ & $246 \pm 031 \mathrm{E}-1$ & $327 \pm 041 \mathrm{E}-1$ & $277 \pm 019 \mathrm{E}-1$ & $266 \pm 011 \mathrm{E}-1$ \\
\hline NGC 3184 & $754 \pm 015 \mathrm{E}-1$ & $103 \pm 005 \mathrm{E}+0$ & $112 \pm 005 \mathrm{E}+0$ & $908 \pm 046 \mathrm{E}-1$ & $556 \pm 075 \mathrm{E}-1$ & $356 \pm 048 \mathrm{E}-1$ & $666 \pm 084 \mathrm{E}-1$ & $143 \pm 017 \mathrm{E}+0$ & $115 \pm 008 \mathrm{E}+0$ & $142 \pm 005 \mathrm{E}+0$ \\
\hline NGC 3198 & $417 \pm 008 \mathrm{E}-1$ & $568 \pm 028 \mathrm{E}-1$ & $627 \pm 031 \mathrm{E}-1$ & $545 \pm 027 \mathrm{E}-1$ & $273 \pm 036 \mathrm{E}-1$ & $173 \pm 023 \mathrm{E}-1$ & $335 \pm 042 \mathrm{E}-1$ & $682 \pm 085 \mathrm{E}-1$ & $605 \pm 042 \mathrm{E}-1$ & $105 \pm 004 \mathrm{E}+0$ \\
\hline IC2574 & $\ldots$ & $360 \pm 020 \mathrm{E}-1$ & $232 \pm 019 \mathrm{E}-1$ & $172 \pm 021 \mathrm{E}-1$ & $124 \pm 020 \mathrm{E}-1$ & $906 \pm 127 \mathrm{E}-2$ & $642 \pm 087 \mathrm{E}-2$ & $688 \pm 089 \mathrm{E}-2$ & $442 \pm 032 \mathrm{E}-2$ & $281 \pm 011 \mathrm{E}-1$ \\
\hline NGC 3265 & $361 \pm 008 \mathrm{E}-2$ & $497 \pm 028 \mathrm{E}-2$ & $565 \pm 035 \mathrm{E}-2$ & $476 \pm 035 \mathrm{E}-2$ & $283 \pm 037 \mathrm{E}-2$ & $198 \pm 026 \mathrm{E}-2$ & $410 \pm 054 \mathrm{E}-2$ & $101 \pm 012 \mathrm{E}-1$ & $855 \pm 059 \mathrm{E}-2$ & $294 \pm 012 \mathrm{E}-1$ \\
\hline Mrk 33 & $384 \pm 008 \mathrm{E}-2$ & $486 \pm 028 \mathrm{E}-2$ & $555 \pm 035 \mathrm{E}-2$ & $476 \pm 035 \mathrm{E}-2$ & $265 \pm 036 \mathrm{E}-2$ & $188 \pm 026 \mathrm{E}-2$ & $528 \pm 067 \mathrm{E}-2$ & $127 \pm 016 \mathrm{E}-1$ & $157 \pm 010 \mathrm{E}-1$ & $861 \pm 035 \mathrm{E}-1$ \\
\hline NGC 3351 & $110 \pm 002 \mathrm{E}+0$ & $164 \pm 008 \mathrm{E}+0$ & $174 \pm 008 \mathrm{E}+0$ & $152 \pm 007 \mathrm{E}+0$ & $770 \pm 109 \mathrm{E}-1$ & $500 \pm 070 \mathrm{E}-1$ & $58 \pm 092 \mathrm{E}-1$ & $126 \pm 016 \mathrm{E}+0$ & $115 \pm 008 \mathrm{E}+0$ & $253 \pm 010 \mathrm{E}+0$ \\
\hline NGC 3521 & $235 \pm 004 \mathrm{E}+0$ & $355 \pm 017 \mathrm{E}+0$ & $408 \pm 020 \mathrm{E}+0$ & $342 \pm 017 \mathrm{E}+0$ & $195 \pm 027 \mathrm{E}+0$ & $129 \pm 018 \mathrm{E}+0$ & $235 \pm 032 \mathrm{E}+0$ & $559 \pm 075 \mathrm{E}+0$ & $483 \pm 033 \mathrm{E}+0$ & $548 \pm 021 \mathrm{E}+0$ \\
\hline NGC 3621 & $\ldots$ & $181 \pm 009 \mathrm{E}+0$ & $205 \pm 010 \mathrm{E}+0$ & $164 \pm 008 \mathrm{E}+0$ & $983 \pm 132 \mathrm{E}-1$ & $664 \pm 091 \mathrm{E}-1$ & $160 \pm 020 \mathrm{E}+0$ & $347 \pm 043 \mathrm{E}+0$ & $293 \pm 020 \mathrm{E}+0$ & $368 \pm 018 \mathrm{E}+0$ \\
\hline NGC 3627 & $236 \pm 004 \mathrm{E}+0$ & $324 \pm 016 \mathrm{E}+0$ & $366 \pm 018 \mathrm{E}+0$ & $313 \pm 015 \mathrm{E}+0$ & $177 \pm 025 \mathrm{E}+0$ & $115 \pm 017 \mathrm{E}+0$ & $219 \pm 030 \mathrm{E}+0$ & $520 \pm 069 \mathrm{E}+0$ & $460 \pm 032 \mathrm{E}+0$ & $750 \pm 029 \mathrm{E}+0$ \\
\hline NGC 3773 & $345 \pm 008 \mathrm{E}-2$ & $442 \pm 023 \mathrm{E}-2$ & $380 \pm 022 \mathrm{E}-2$ & $370 \pm 023 \mathrm{E}-2$ & $221 \pm 027 \mathrm{E}-2$ & $143 \pm 023 \mathrm{E}-2$ & $255 \pm 034 \mathrm{E}-2$ & $474 \pm 059 \mathrm{E}-2$ & $409 \pm 028 \mathrm{E}-2$ & $144 \pm 005 \mathrm{E}-1$ \\
\hline NGC 3938 & $464 \pm 009 \mathrm{E}-1$ & $625 \pm 031 \mathrm{E}-1$ & $570 \pm 029 \mathrm{E}-1$ & $531 \pm 027 \mathrm{E}-1$ & $320 \pm 043 \mathrm{E}-1$ & $211 \pm 029 \mathrm{E}-1$ & $410 \pm 052 \mathrm{E}-1$ & $980 \pm 122 \mathrm{E}-1$ & $846 \pm 059 \mathrm{E}-1$ & $108 \pm 004 \mathrm{E}+0$ \\
\hline NGC 4125 & $795 \pm 016 \mathrm{E}-1$ & $136 \pm 006 \mathrm{E}+0$ & $151 \pm 007 \mathrm{E}+0$ & $127 \pm 006 \mathrm{E}+0$ & $639 \pm 086 \mathrm{E}-1$ & $371 \pm 050 \mathrm{E}-1$ & $247 \pm 032 \mathrm{E}-1$ & $143 \pm 017 \mathrm{E}-1$ & $104 \pm 007 \mathrm{E}-1$ & $784 \pm 042 \mathrm{E}-2$ \\
\hline NGC 4236 & $\ldots$ & $626 \pm 033 \mathrm{E}-1$ & $824 \pm 044 \mathrm{E}-1$ & $566 \pm 034 \mathrm{E}-1$ & $245 \pm 033 \mathrm{E}-1$ & $185 \pm 028 \mathrm{E}-1$ & $184 \pm 014 \mathrm{E}-1$ & $215 \pm 027 \mathrm{E}-1$ & $138 \pm 009 \mathrm{E}-1$ & $513 \pm 020 \mathrm{E}-1$ \\
\hline
\end{tabular}


Table 2

(Continued)

\begin{tabular}{|c|c|c|c|c|c|c|c|c|c|c|}
\hline Filter & $\begin{array}{c}\text { SDSS } \\
z\end{array}$ & $\begin{array}{c}\text { 2MASS } \\
J\end{array}$ & $\begin{array}{c}\text { 2MASS } \\
H\end{array}$ & $\begin{array}{c}2 \mathrm{MASS} \\
K_{\mathrm{s}}\end{array}$ & $\begin{array}{l}\text { Spitzer } \\
\text { IRAC }\end{array}$ & $\begin{array}{l}\text { Spitzer } \\
\text { IRAC }\end{array}$ & $\begin{array}{l}\text { Spitzer } \\
\text { IRAC }\end{array}$ & $\begin{array}{l}\text { Spitzer } \\
\text { IRAC }\end{array}$ & $\begin{array}{l}\text { WISE } \\
\text { W3 }\end{array}$ & $\begin{array}{l}\text { Spitzer } \\
\text { MIPS }\end{array}$ \\
\hline $\bar{\lambda}(\mu \mathrm{m})$ & 0.910 & 1.24 & 1.65 & 2.17 & 3.56 & 4.51 & 5.76 & 7.96 & 12.8 & 23.8 \\
\hline$\Delta \lambda(\mu \mathrm{m})$ & 0.131 & 0.163 & 0.252 & 0.264 & 0.687 & 0.872 & 1.25 & 2.55 & 5.51 & 5.32 \\
\hline$A_{\lambda} / A_{V}$ & 0.488 & 0.296 & 0.187 & 0.116 & 0.0451 & 0.0288 & 0.0193 & 0.0296 & 0.0355 & 0.0193 \\
\hline NGC 4254 & $842 \pm 017 \mathrm{E}-1$ & $122 \pm 006 \mathrm{E}+0$ & $132 \pm 006 \mathrm{E}+0$ & $119 \pm 006 \mathrm{E}+0$ & $697 \pm 094 \mathrm{E}-1$ & $468 \pm 064 \mathrm{E}-1$ & $149 \pm 018 \mathrm{E}+0$ & $392 \pm 048 \mathrm{E}+0$ & $331 \pm 023 \mathrm{E}+0$ & $419 \pm 016 \mathrm{E}+0$ \\
\hline NGC 4321 & $122 \pm 002 \mathrm{E}+0$ & $182 \pm 009 \mathrm{E}+0$ & $197 \pm 009 \mathrm{E}+0$ & $163 \pm 008 \mathrm{E}+0$ & $950 \pm 128 \mathrm{E}-1$ & $637 \pm 087 \mathrm{E}-1$ & $121 \pm 015 \mathrm{E}+0$ & $287 \pm 035 \mathrm{E}+0$ & $257 \pm 018 \mathrm{E}+0$ & $333 \pm 013 \mathrm{E}+0$ \\
\hline NGC 4450 & $789 \pm 015 \mathrm{E}-1$ & $117 \pm 005 \mathrm{E}+0$ & $136 \pm 006 \mathrm{E}+0$ & $106 \pm 005 \mathrm{E}+0$ & $527 \pm 071 \mathrm{E}-1$ & $324 \pm 044 \mathrm{E}-1$ & $260 \pm 033 \mathrm{E}-1$ & $267 \pm 033 \mathrm{E}-1$ & $246 \pm 017 \mathrm{E}-1$ & $209 \pm 011 \mathrm{E}-1$ \\
\hline NGC 4536 & $491 \pm 010 \mathrm{E}-1$ & $702 \pm 035 \mathrm{E}-1$ & $742 \pm 037 \mathrm{E}-1$ & $694 \pm 035 \mathrm{E}-1$ & $394 \pm 053 \mathrm{E}-1$ & $285 \pm 038 \mathrm{E}-1$ & $620 \pm 079 \mathrm{E}-1$ & $165 \pm 020 \mathrm{E}+0$ & $131 \pm 009 \mathrm{E}+0$ & $345 \pm 013 \mathrm{E}+0$ \\
\hline NGC 4552 & $988 \pm 019 \mathrm{E}-1$ & $157 \pm 007 \mathrm{E}+0$ & $175 \pm 008 \mathrm{E}+0$ & $143 \pm 007 \mathrm{E}+0$ & $824 \pm 111 \mathrm{E}-1$ & $480 \pm 065 \mathrm{E}-1$ & $299 \pm 038 \mathrm{E}-1$ & $170 \pm 021 \mathrm{E}-1$ & $100 \pm 007 \mathrm{E}-1$ & $940 \pm 041 \mathrm{E}-2$ \\
\hline NGC 4559 & $545 \pm 011 \mathrm{E}-1$ & $760 \pm 038 \mathrm{E}-1$ & $777 \pm 039 \mathrm{E}-1$ & $652 \pm 033 \mathrm{E}-1$ & $353 \pm 047 \mathrm{E}-1$ & $233 \pm 032 \mathrm{E}-1$ & $420 \pm 053 \mathrm{E}-1$ & $837 \pm 104 \mathrm{E}-1$ & $629 \pm 044 \mathrm{E}-1$ & $111 \pm 004 \mathrm{E}+0$ \\
\hline NGC 4569 & $108 \pm 002 \mathrm{E}+0$ & $175 \pm 008 \mathrm{E}+0$ & $203 \pm 010 \mathrm{E}+0$ & $164 \pm 008 \mathrm{E}+0$ & $759 \pm 102 \mathrm{E}-1$ & $472 \pm 064 \mathrm{E}-1$ & $590 \pm 075 \mathrm{E}-1$ & $101 \pm 012 \mathrm{E}+0$ & $931 \pm 065 \mathrm{E}-1$ & $143 \pm 005 \mathrm{E}+0$ \\
\hline NGC 4579 & $111 \pm 002 \mathrm{E}+0$ & $198 \pm 009 \mathrm{E}+0$ & $218 \pm 010 \mathrm{E}+0$ & $179 \pm 008 \mathrm{E}+0$ & $863 \pm 117 \mathrm{E}-1$ & $520 \pm 071 \mathrm{E}-1$ & $541 \pm 069 \mathrm{E}-1$ & $726 \pm 090 \mathrm{E}-1$ & $706 \pm 049 \mathrm{E}-1$ & $759 \pm 031 \mathrm{E}-1$ \\
\hline NGC 4594 & $523 \pm 010 \mathrm{E}+0$ & $771 \pm 038 \mathrm{E}+0$ & $894 \pm 044 \mathrm{E}+0$ & $743 \pm 037 \mathrm{E}+0$ & $389 \pm 053 \mathrm{E}+0$ & $235 \pm 031 \mathrm{E}+0$ & $179 \pm 022 \mathrm{E}+0$ & $144 \pm 016 \mathrm{E}+0$ & $109 \pm 007 \mathrm{E}+0$ & $771 \pm 030 \mathrm{E}-1$ \\
\hline NGC 4625 & $795 \pm 017 \mathrm{E}-2$ & $966 \pm 063 \mathrm{E}-2$ & $112 \pm 008 \mathrm{E}-1$ & $892 \pm 087 \mathrm{E}-2$ & $486 \pm 064 \mathrm{E}-2$ & $307 \pm 040 \mathrm{E}-2$ & $605 \pm 076 \mathrm{E}-2$ & $134 \pm 016 \mathrm{E}-1$ & $112 \pm 007 \mathrm{E}-1$ & $128 \pm 005 \mathrm{E}-1$ \\
\hline NGC 4631 & $121 \pm 002 \mathrm{E}+0$ & $172 \pm 008 \mathrm{E}+0$ & $196 \pm 009 \mathrm{E}+0$ & $182 \pm 009 \mathrm{E}+0$ & $119 \pm 017 \mathrm{E}+0$ & $845 \pm 114 \mathrm{E}-1$ & $247 \pm 031 \mathrm{E}+0$ & $584 \pm 072 \mathrm{E}+0$ & $463 \pm 032 \mathrm{E}+0$ & $812 \pm 032 \mathrm{E}+0$ \\
\hline NGC 4725 & $159 \pm 003 \mathrm{E}+0$ & $240 \pm 012 \mathrm{E}+0$ & $315 \pm 015 \mathrm{E}+0$ & $239 \pm 012 \mathrm{E}+0$ & $113 \pm 015 \mathrm{E}+0$ & $704 \pm 096 \mathrm{E}-1$ & $753 \pm 095 \mathrm{E}-1$ & $120 \pm 015 \mathrm{E}+0$ & $875 \pm 061 \mathrm{E}-1$ & $859 \pm 037 \mathrm{E}-1$ \\
\hline NGC 4736 & $518 \pm 010 \mathrm{E}+0$ & $684 \pm 034 \mathrm{E}+0$ & $760 \pm 038 \mathrm{E}+0$ & $639 \pm 032 \mathrm{E}+0$ & $344 \pm 048 \mathrm{E}+0$ & $229 \pm 031 \mathrm{E}+0$ & $256 \pm 034 \mathrm{E}+0$ & $481 \pm 064 \mathrm{E}+0$ & $454 \pm 031 \mathrm{E}+0$ & $552 \pm 022 \mathrm{E}+0$ \\
\hline DDO 154 & $126 \pm 004 \mathrm{E}-2$ & $984 \pm 260 \mathrm{E}-3$ & $123 \pm 039 \mathrm{E}-2$ & $119 \pm 047 \mathrm{E}-2$ & $510 \pm 100 \mathrm{E}-3$ & $350 \pm 100 \mathrm{E}-3$ & $<403 \mathrm{E}-3$ & $<399 \mathrm{E}-3$ & $<124 \mathrm{E}-3$ & $<438 \mathrm{E}-3$ \\
\hline NGC 4826 & $336 \pm 006 \mathrm{E}+0$ & $547 \pm 027 \mathrm{E}+0$ & $616 \pm 030 \mathrm{E}+0$ & $520 \pm 026 \mathrm{E}+0$ & $239 \pm 034 \mathrm{E}+0$ & $151 \pm 021 \mathrm{E}+0$ & $159 \pm 021 \mathrm{E}+0$ & $223 \pm 029 \mathrm{E}+0$ & $185 \pm 012 \mathrm{E}+0$ & $254 \pm 010 \mathrm{E}+0$ \\
\hline DDO 165 & $306 \pm 008 \mathrm{E}-2$ & $313 \pm 035 \mathrm{E}-2$ & $463 \pm 054 \mathrm{E}-2$ & $370 \pm 062 \mathrm{E}-2$ & $127 \pm 023 \mathrm{E}-2$ & $907 \pm 150 \mathrm{E}-3$ & $587 \pm 170 \mathrm{E}-3$ & $407 \pm 080 \mathrm{E}-3$ & $<168 \mathrm{E}-3$ & $<448 \mathrm{E}-3$ \\
\hline NGC 5033 & $720 \pm 014 \mathrm{E}-1$ & $119 \pm 006 \mathrm{E}+0$ & $133 \pm 006 \mathrm{E}+0$ & $116 \pm 005 \mathrm{E}+0$ & $639 \pm 086 \mathrm{E}-1$ & $470 \pm 064 \mathrm{E}-1$ & $815 \pm 103 \mathrm{E}-1$ & $192 \pm 023 \mathrm{E}+0$ & $172 \pm 012 \mathrm{E}+0$ & $196 \pm 007 \mathrm{E}+0$ \\
\hline NGC 5055 & $309 \pm 006 \mathrm{E}+0$ & $414 \pm 020 \mathrm{E}+0$ & $491 \pm 024 \mathrm{E}+0$ & $402 \pm 020 \mathrm{E}+0$ & $236 \pm 032 \mathrm{E}+0$ & $155 \pm 021 \mathrm{E}+0$ & $259 \pm 033 \mathrm{E}+0$ & $558 \pm 070 \mathrm{E}+0$ & $524 \pm 036 \mathrm{E}+0$ & $558 \pm 022 \mathrm{E}+0$ \\
\hline NGC 5194 & $414 \pm 008 \mathrm{E}+0$ & $484 \pm 024 \mathrm{E}+0$ & $577 \pm 029 \mathrm{E}+0$ & $446 \pm 022 \mathrm{E}+0$ & $265 \pm 035 \mathrm{E}+0$ & $178 \pm 025 \mathrm{E}+0$ & $422 \pm 053 \mathrm{E}+0$ & $106 \pm 013 \mathrm{E}+1$ & $101 \pm 007 \mathrm{E}+1$ & $124 \pm 004 \mathrm{E}+1$ \\
\hline NGC 5195 & $112 \pm 002 \mathrm{E}+0$ & $229 \pm 011 \mathrm{E}+0$ & $274 \pm 013 \mathrm{E}+0$ & $222 \pm 011 \mathrm{E}+0$ & $830 \pm 113 \mathrm{E}-1$ & $509 \pm 070 \mathrm{E}-1$ & $461 \pm 060 \mathrm{E}-1$ & $644 \pm 080 \mathrm{E}-1$ & $997 \pm 069 \mathrm{E}-1$ & $146 \pm 005 \mathrm{E}+0$ \\
\hline NGC 5398 & & $767 \pm 040 \mathrm{E}-2$ & $644 \pm 037 \mathrm{E}-2$ & $527 \pm 035 \mathrm{E}-2$ & $375 \pm 050 \mathrm{E}-2$ & $245 \pm 036 \mathrm{E}-2$ & $143 \pm 020 \mathrm{E}-2$ & $585 \pm 075 \mathrm{E}-2$ & $641 \pm 044 \mathrm{E}-2$ & $278 \pm 013 \mathrm{E}-1$ \\
\hline NGC 5457 & $386 \pm 007 \mathrm{E}+0$ & $435 \pm 021 \mathrm{E}+0$ & $501 \pm 025 \mathrm{E}+0$ & $440 \pm 022 \mathrm{E}+0$ & $281 \pm 038 \mathrm{E}+0$ & $189 \pm 025 \mathrm{E}+0$ & $338 \pm 042 \mathrm{E}+0$ & $761 \pm 094 \mathrm{E}+0$ & $687 \pm 048 \mathrm{E}+0$ & $105 \pm 004 \mathrm{E}+1$ \\
\hline NGC 5408 & & $124 \pm 006 \mathrm{E}-1$ & $116 \pm 006 \mathrm{E}-1$ & $742 \pm 050 \mathrm{E}-2$ & $376 \pm 071 \mathrm{E}-2$ & $214 \pm 051 \mathrm{E}-2$ & $284 \pm 053 \mathrm{E}-2$ & $254 \pm 047 \mathrm{E}-2$ & $645 \pm 045 \mathrm{E}-2$ & $427 \pm 017 \mathrm{E}-1$ \\
\hline NGC 5474 & $222 \pm 004 \mathrm{E}-1$ & $262 \pm 014 \mathrm{E}-1$ & $305 \pm 018 \mathrm{E}-1$ & $193 \pm 015 \mathrm{E}-1$ & $108 \pm 013 \mathrm{E}-1$ & $730 \pm 102 \mathrm{E}-2$ & $555 \pm 101 \mathrm{E}-2$ & $114 \pm 014 \mathrm{E}-1$ & $100 \pm 007 \mathrm{E}-1$ & $156 \pm 006 \mathrm{E}-1$ \\
\hline NGC 5713 & $230 \pm 004 \mathrm{E}-1$ & $362 \pm 018 \mathrm{E}-1$ & $379 \pm 019 \mathrm{E}-1$ & $327 \pm 016 \mathrm{E}-1$ & $200 \pm 027 \mathrm{E}-1$ & $137 \pm 019 \mathrm{E}-1$ & $288 \pm 037 \mathrm{E}-1$ & $114 \pm 014 \mathrm{E}+0$ & $982 \pm 068 \mathrm{E}-1$ & $234 \pm 009 \mathrm{E}+0$ \\
\hline NGC 5866 & $889 \pm 017 \mathrm{E}-1$ & $129 \pm 006 \mathrm{E}+0$ & $147 \pm 007 \mathrm{E}+0$ & $125 \pm 006 \mathrm{E}+0$ & $662 \pm 089 \mathrm{E}-1$ & $420 \pm 057 \mathrm{E}-1$ & $311 \pm 039 \mathrm{E}-1$ & $314 \pm 039 \mathrm{E}-1$ & $260 \pm 018 \mathrm{E}-1$ & $213 \pm 008 \mathrm{E}-1$ \\
\hline IC4710 & $\cdots$ & $997 \pm 054 \mathrm{E}-2$ & $951 \pm 057 \mathrm{E}-2$ & $753 \pm 054 \mathrm{E}-2$ & $693 \pm 096 \mathrm{E}-2$ & $461 \pm 064 \mathrm{E}-2$ & $448 \pm 061 \mathrm{E}-2$ & $644 \pm 082 \mathrm{E}-2$ & $362 \pm 025 \mathrm{E}-2$ & $118 \pm 005 \mathrm{E}-1$ \\
\hline NGC 6822 & $\ldots$ & $177 \pm 008 \mathrm{E}+0$ & $184 \pm 009 \mathrm{E}+0$ & $146 \pm 007 \mathrm{E}+0$ & $157 \pm 027 \mathrm{E}+0$ & $973 \pm 185 \mathrm{E}-1$ & $988 \pm 179 \mathrm{E}-1$ & $122 \pm 017 \mathrm{E}+0$ & $110 \pm 007 \mathrm{E}+0$ & $313 \pm 012 \mathrm{E}+0$ \\
\hline NGC 6946 & & $546 \pm 027 \mathrm{E}+0$ & $454 \pm 022 \mathrm{E}+0$ & $502 \pm 025 \mathrm{E}+0$ & $315 \pm 042 \mathrm{E}+0$ & $212 \pm 029 \mathrm{E}+0$ & $576 \pm 072 \mathrm{E}+0$ & $137 \pm 017 \mathrm{E}+1$ & $127 \pm 008 \mathrm{E}+1$ & $199 \pm 007 \mathrm{E}+1$ \\
\hline NGC 7331 & $169 \pm 003 \mathrm{E}+0$ & $264 \pm 013 \mathrm{E}+0$ & $320 \pm 016 \mathrm{E}+0$ & $273 \pm 013 \mathrm{E}+0$ & $158 \pm 021 \mathrm{E}+0$ & $101 \pm 013 \mathrm{E}+0$ & $185 \pm 023 \mathrm{E}+0$ & $401 \pm 049 \mathrm{E}+0$ & $335 \pm 023 \mathrm{E}+0$ & $434 \pm 024 \mathrm{E}+0$ \\
\hline NGC 7552 & $\cdots$ & $699 \pm 035 \mathrm{E}-1$ & $791 \pm 039 \mathrm{E}-1$ & $694 \pm 035 \mathrm{E}-1$ & $452 \pm 061 \mathrm{E}-1$ & $360 \pm 049 \mathrm{E}-1$ & $106 \pm 013 \mathrm{E}+0$ & $270 \pm 033 \mathrm{E}+0$ & $266 \pm 018 \mathrm{E}+0$ & $106 \pm 004 \mathrm{E}+1$ \\
\hline \multirow[t]{2}{*}{ NGC 7793} & $\cdots$ & $164 \pm 008 \mathrm{E}+0$ & $168 \pm 008 \mathrm{E}+0$ & $130 \pm 006 \mathrm{E}+0$ & $744 \pm 103 \mathrm{E}-1$ & $481 \pm 064 \mathrm{E}-1$ & $104 \pm 013 \mathrm{E}+0$ & $189 \pm 023 \mathrm{E}+0$ & $147 \pm 010 \mathrm{E}+0$ & $209 \pm 008 \mathrm{E}+0$ \\
\hline & Spitzer & Herschel & Herschel & Spitzer & Herschel & Herschel & Herschel & Herschel & JCMT & Planck \\
\hline Filter & MIPS & PACS & PACS & MIPS & PACS & SPIRE & SPIRE & SPIRE & SCUBA & HFI \\
\hline $\bar{\lambda}(\mu \mathrm{m})$ & 72.5 & 71.8 & 103 & 157 & 167 & 252 & 353 & 511 & 850 & 850 \\
\hline$\Delta \lambda(\mu \mathrm{m})$ & 21.3 & 21.3 & 30.6 & 35.8 & 68.5 & 67.3 & 95.2 & 184 & 247 & 238 \\
\hline$A_{\lambda} / A_{V}$ & 0.00231 & 0.00231 & 0.00104 & 0.000388 & 0.000388 & 0.000152 & 0.0000725 & 0.0000361 & 0.0000146 & 0.0 \\
\hline NGC 0024 & $222 \pm 015 \mathrm{E}+0$ & $\cdots$ & $\cdots$ & $712 \pm 085 \mathrm{E}+0$ & $\ldots$ & $\cdots$ & $\cdots$ & $\cdots$ & $\cdots$ & $457 \pm 091 \mathrm{E}-1$ \\
\hline NGC 0337 & $111 \pm 007 \mathrm{E}+1$ & $139 \pm 006 \mathrm{E}+1$ & $210 \pm 010 \mathrm{E}+1$ & $200 \pm 024 \mathrm{E}+1$ & $197 \pm 009 \mathrm{E}+1$ & $894 \pm 063 \mathrm{E}+0$ & $405 \pm 028 \mathrm{E}+0$ & $158 \pm 011 \mathrm{E}+0$ & $349 \pm 052 \mathrm{E}-1$ & $573 \pm 234 \mathrm{E}-1$ \\
\hline NGC 0584 & $175 \pm 045 \mathrm{E}-1$ & $180 \pm 025 \mathrm{E}-1$ & $300 \pm 033 \mathrm{E}-1$ & $118 \pm 030 \mathrm{E}+0$ & $610 \pm 038 \mathrm{E}-1$ & $<838 \mathrm{E}-1$ & $<603 \mathrm{E}-1$ & $<419 \mathrm{E}-1$ & $\ldots$ & $\ldots$ \\
\hline NGC 0628 & $338 \pm 023 \mathrm{E}+1$ & $420 \pm 021 \mathrm{E}+1$ & $836 \pm 041 \mathrm{E}+1$ & $111 \pm 013 \mathrm{E}+2$ & $114 \pm 005 \mathrm{E}+2$ & $621 \pm 044 \mathrm{E}+1$ & $298 \pm 021 \mathrm{E}+1$ & $114 \pm 008 \mathrm{E}+1$ & $\cdots$ & $198 \pm 015 \mathrm{E}+0$ \\
\hline NGC 0855 & $168 \pm 011 \mathrm{E}+0$ & $208 \pm 010 \mathrm{E}+0$ & $253 \pm 013 \mathrm{E}+0$ & $222 \pm 026 \mathrm{E}+0$ & $206 \pm 010 \mathrm{E}+0$ & $135 \pm 009 \mathrm{E}+0$ & $726 \pm 052 \mathrm{E}-1$ & $222 \pm 018 \mathrm{E}-1$ & $\cdots$ & $\cdots$ \\
\hline NGC 0925 & $143 \pm 010 \mathrm{E}+1$ & $136 \pm 006 \mathrm{E}+1$ & $289 \pm 014 \mathrm{E}+1$ & $433 \pm 052 \mathrm{E}+1$ & $372 \pm 018 \mathrm{E}+1$ & $251 \pm 017 \mathrm{E}+1$ & $138 \pm 009 \mathrm{E}+1$ & $652 \pm 046 \mathrm{E}+0$ & $\cdots$ & $\cdots$ \\
\hline
\end{tabular}


Table 2

(Continued)

\begin{tabular}{|c|c|c|c|c|c|c|c|c|c|c|}
\hline Filter & $\begin{array}{l}\text { Spitzer } \\
\text { MIPS }\end{array}$ & $\begin{array}{c}\text { Herschel } \\
\text { PACS }\end{array}$ & $\begin{array}{c}\text { Herschel } \\
\text { PACS }\end{array}$ & $\begin{array}{l}\text { Spitzer } \\
\text { MIPS }\end{array}$ & $\begin{array}{c}\text { Herschel } \\
\text { PACS }\end{array}$ & $\begin{array}{c}\text { Herschel } \\
\text { SPIRE }\end{array}$ & $\begin{array}{c}\text { Herschel } \\
\text { SPIRE }\end{array}$ & $\begin{array}{c}\text { Herschel } \\
\text { SPIRE }\end{array}$ & $\begin{array}{c}\text { JCMT } \\
\text { SCUBA }\end{array}$ & $\begin{array}{c}\text { Planck } \\
\text { HFI }\end{array}$ \\
\hline $\bar{\lambda}(\mu \mathrm{m})$ & 72.5 & 71.8 & 103 & 157 & 167 & 252 & 353 & 511 & 850 & 850 \\
\hline$\Delta \lambda(\mu \mathrm{m})$ & 21.3 & 21.3 & 30.6 & 35.8 & 68.5 & 67.3 & 95.2 & 184 & 247 & 238 \\
\hline$A_{\lambda} / A_{V}$ & 0.00231 & 0.00231 & 0.00104 & 0.000388 & 0.000388 & 0.000152 & 0.0000725 & 0.0000361 & 0.0000146 & 0.0 \\
\hline NGC 1097 & $598 \pm 046 \mathrm{E}+1$ & $787 \pm 039 \mathrm{E}+1$ & $123 \pm 006 \mathrm{E}+2$ & $153 \pm 018 \mathrm{E}+2$ & $132 \pm 006 \mathrm{E}+2$ & $669 \pm 047 \mathrm{E}+1$ & $293 \pm 020 \mathrm{E}+1$ & $106 \pm 007 \mathrm{E}+1$ & $144 \pm 078 \mathrm{E}+0$ & $212 \pm 012 \mathrm{E}+0$ \\
\hline NGC 1266 & $126 \pm 009 \mathrm{E}+1$ & $148 \pm 007 \mathrm{E}+1$ & $171 \pm 008 \mathrm{E}+1$ & $102 \pm 012 \mathrm{E}+1$ & $113 \pm 005 \mathrm{E}+1$ & $404 \pm 028 \mathrm{E}+0$ & $145 \pm 010 \mathrm{E}+0$ & $402 \pm 030 \mathrm{E}-1$ & $\ldots$ & $\ldots$ \\
\hline NGC 1291 & $527 \pm 037 \mathrm{E}+0$ & $439 \pm 027 \mathrm{E}+0$ & $135 \pm 007 \mathrm{E}+1$ & $262 \pm 031 \mathrm{E}+1$ & $226 \pm 011 \mathrm{E}+1$ & $151 \pm 010 \mathrm{E}+1$ & $757 \pm 054 \mathrm{E}+0$ & $309 \pm 022 \mathrm{E}+0$ & $\ldots$ & $\ldots$ \\
\hline NGC 1316 & $543 \pm 040 \mathrm{E}+0$ & $568 \pm 029 \mathrm{E}+0$ & $997 \pm 050 \mathrm{E}+0$ & $126 \pm 017 \mathrm{E}+1$ & $123 \pm 006 \mathrm{E}+1$ & $494 \pm 035 \mathrm{E}+0$ & $204 \pm 015 \mathrm{E}+0$ & $707 \pm 058 \mathrm{E}-1$ & $\ldots$ & $\ldots$ \\
\hline NGC 1377 & $634 \pm 046 \mathrm{E}+0$ & $725 \pm 036 \mathrm{E}+0$ & $651 \pm 032 \mathrm{E}+0$ & $338 \pm 042 \mathrm{E}+0$ & $337 \pm 016 \mathrm{E}+0$ & $122 \pm 008 \mathrm{E}+0$ & $469 \pm 034 \mathrm{E}-1$ & $163 \pm 013 \mathrm{E}-1$ & $\ldots$ & $\ldots$ \\
\hline NGC 1404 & $<165 \mathrm{E}-1$ & $<214 \mathrm{E}-1$ & $<252 \mathrm{E}-1$ & $<286 \mathrm{E}-1$ & $<204 \mathrm{E}-1$ & $<154 \mathrm{E}-1$ & $<134 \mathrm{E}-1$ & $<909 \mathrm{E}-2$ & $\ldots$ & $\ldots$ \\
\hline IC0342 & $351 \pm 024 \mathrm{E}+2$ & $470 \pm 023 \mathrm{E}+2$ & $894 \pm 044 \mathrm{E}+2$ & $914 \pm 109 \mathrm{E}+2$ & $107 \pm 005 E+3$ & $564 \pm 040 \mathrm{E}+2$ & $51 \pm 017 \mathrm{E}+2$ & $922 \pm 065 \mathrm{E}+1$ & $\ldots$ & $\ldots$ \\
\hline NGC 1482 & $324 \pm 028 \mathrm{E}+1$ & $419 \pm 020 \mathrm{E}+1$ & $522 \pm 026 \mathrm{E}+1$ & $387 \pm 046 \mathrm{E}+1$ & $419 \pm 021 \mathrm{E}+1$ & $154 \pm 010 \mathrm{E}+1$ & $32 \pm 041 \mathrm{E}+0$ & $176 \pm 012 \mathrm{E}+0$ & $30 \pm 050 \mathrm{E}-1$ & $\ldots$ \\
\hline NGC 1512 & $682 \pm 048 \mathrm{E}+0$ & $752 \pm 039 \mathrm{E}+0$ & $150 \pm 007 \mathrm{E}+1$ & $196 \pm 023 \mathrm{E}+1$ & $191 \pm 009 \mathrm{E}+1$ & $147 \pm 010 \mathrm{E}+1$ & $866 \pm 062 \mathrm{E}+0$ & $363 \pm 026 \mathrm{E}+0$ & $\ldots$ & $551 \pm 090 \mathrm{E}-1$ \\
\hline NGC 1566 & $343 \pm 025 \mathrm{E}+1$ & $\ldots$ & $\ldots$ & $102 \pm 012 \mathrm{E}+2$ & $\ldots$ & $\ldots$ & $\ldots$ & $\ldots$ & $\ldots$ & $142 \pm 010 \mathrm{E}+0$ \\
\hline NGC 1705 & $124 \pm 008 \mathrm{E}+0$ & $\ldots$ & $\ldots$ & $139 \pm 016 \mathrm{E}+0$ & $\ldots$ & $\ldots$ & $\ldots$ & $\ldots$ & $\ldots$ & $\ldots$ \\
\hline NGC 2146 & $215 \pm 015 \mathrm{E}+2$ & $198 \pm 009 \mathrm{E}+2$ & $236 \pm 011 \mathrm{E}+2$ & $119 \pm 014 \mathrm{E}+2$ & $174 \pm 008 \mathrm{E}+2$ & $613 \pm 043 \mathrm{E}+1$ & $221 \pm 015 \mathrm{E}+1$ & $681 \pm 048 \mathrm{E}+0$ & $\ldots$ & $\ldots$ \\
\hline NGC 2403 & $857 \pm 060 \mathrm{E}+1$ & $994 \pm 049 \mathrm{E}+1$ & $\ldots$ & $225 \pm 027 \mathrm{E}+2$ & $192 \pm 009 \mathrm{E}+2$ & $119 \pm 008 \mathrm{E}+2$ & $627 \pm 048 \mathrm{E}+1$ & $279 \pm 024 \mathrm{E}+1$ & $\ldots$ & $422 \pm 032 \mathrm{E}+0$ \\
\hline HoII & $318 \pm 022 \mathrm{E}+0$ & $438 \pm 022 \mathrm{E}+0$ & $574 \pm 028 \mathrm{E}+0$ & $346 \pm 042 \mathrm{E}+0$ & $363 \pm 018 \mathrm{E}+0$ & $152 \pm 011 \mathrm{E}+0$ & $771 \pm 067 \mathrm{E}-1$ & $284 \pm 142 \mathrm{E}-1$ & $\ldots$ & $\ldots$ \\
\hline M81dwA & $<149 \mathrm{E}-1$ & $\ldots$ & $\ldots$ & $<143 \mathrm{E}-1$ & $\ldots$ & $\ldots$ & $\ldots$ & $\ldots$ & $\ldots$ & $\ldots$ \\
\hline DDO 053 & $315 \pm 025 \mathrm{E}-1$ & $400 \pm 025 \mathrm{E}-1$ & $519 \pm 031 \mathrm{E}-1$ & $357 \pm 050 \mathrm{E}-1$ & $300 \pm 020 \mathrm{E}-1$ & $138 \pm 014 \mathrm{E}-1$ & $989 \pm 106 \mathrm{E}-2$ & $<389 \mathrm{E}-2$ & $\ldots$ & $\ldots$ \\
\hline NGC 2798 & $217 \pm 017 \mathrm{E}+1$ & $250 \pm 012 \mathrm{E}+1$ & $287 \pm 014 \mathrm{E}+1$ & $206 \pm 024 \mathrm{E}+1$ & $202 \pm 010 \mathrm{E}+1$ & $740 \pm 052 \mathrm{E}+0$ & $272 \pm 019 \mathrm{E}+0$ & $853 \pm 061 \mathrm{E}-1$ & $194 \pm 032 \mathrm{E}-1$ & $\ldots$ \\
\hline NGC 2841 & $102 \pm 007 \mathrm{E}+1$ & $110 \pm 005 \mathrm{E}+1$ & $286 \pm 014 \mathrm{E}+1$ & $622 \pm 075 \mathrm{E}+1$ & $488 \pm 024 \mathrm{E}+1$ & $321 \pm 022 \mathrm{E}+1$ & $152 \pm 010 \mathrm{E}+1$ & $589 \pm 041 \mathrm{E}+0$ & $\ldots$ & $122 \pm 010 \mathrm{E}+0$ \\
\hline NGC 2915 & $140 \pm 010 \mathrm{E}+0$ & $107 \pm 005 \mathrm{E}+0$ & $180 \pm 009 \mathrm{E}+0$ & $145 \pm 026 \mathrm{E}+0$ & $162 \pm 008 \mathrm{E}+0$ & $816 \pm 059 \mathrm{E}-1$ & $463 \pm 034 \mathrm{E}-1$ & $204 \pm 016 \mathrm{E}-1$ & $\ldots$ & $\ldots$ \\
\hline $\mathrm{HoI}$ & $292 \pm 028 \mathrm{E}-1$ & $280 \pm 031 \mathrm{E}-1$ & $41 \pm 039 \mathrm{E}-1$ & $526 \pm 075 \mathrm{E}-1$ & $552 \pm 039 \mathrm{E}-1$ & $369 \pm 031 \mathrm{E}-1$ & $229 \pm 020 \mathrm{E}-1$ & $108 \pm 012 \mathrm{E}-1$ & $\ldots$ & $\ldots$ \\
\hline NGC 2976 & $199 \pm 013 \mathrm{E}+1$ & $209 \pm 010 \mathrm{E}+1$ & $372 \pm 018 \mathrm{E}+1$ & $426 \pm 051 \mathrm{E}+1$ & $459 \pm 022 \mathrm{E}+1$ & $242 \pm 017 \mathrm{E}+1$ & $113 \pm 008 \mathrm{E}+1$ & $442 \pm 031 \mathrm{E}+0$ & $609 \pm 236 \mathrm{E}-1$ & $110 \pm 022 \mathrm{E}+0$ \\
\hline NGC 3049 & $289 \pm 021 \mathrm{E}+0$ & $382 \pm 019 \mathrm{E}+0$ & $529 \pm 026 \mathrm{E}+0$ & $486 \pm 059 \mathrm{E}+0$ & $493 \pm 024 \mathrm{E}+0$ & $259 \pm 018 \mathrm{E}+0$ & $131 \pm 009 \mathrm{E}+0$ & $644 \pm 046 \mathrm{E}-1$ & $\ldots$ & $\ldots$ \\
\hline NGC 3031 & $852 \pm 059 \mathrm{E}+1$ & $997 \pm 049 \mathrm{E}+1$ & $\ldots$ & $308 \pm 037 \mathrm{E}+2$ & $281 \pm 014 \mathrm{E}+2$ & $176 \pm 013 \mathrm{E}+2$ & $876 \pm 070 \mathrm{E}+1$ & $364 \pm 034 \mathrm{E}+1$ & $\ldots$ & $\ldots$ \\
\hline NGC 3034 & $162 \pm 048 \mathrm{E}+3^{\mathrm{a}}$ & $209 \pm 010 \mathrm{E}+3$ & $\ldots$ & $857 \pm 257 \mathrm{E}+2^{\mathrm{a}}$ & $142 \pm 007 \mathrm{E}+3$ & $442 \pm 031 \mathrm{E}+2$ & $151 \pm 010 \mathrm{E}+2$ & $456 \pm 033 \mathrm{E}+1$ & $551 \pm 082 \mathrm{E}+0$ & $900 \pm 018 \mathrm{E}+0$ \\
\hline HoIX & $<228 \mathrm{E}-1$ & $\cdots$ & $\ldots$ & $<457 \mathrm{E}-1$ & $\cdots$ & & & $\cdots$ & $\ldots$ & $\ldots$ \\
\hline NGC 3077 & $196 \pm 013 \mathrm{E}+1$ & $213 \pm 010 \mathrm{E}+1$ & $292 \pm 014 \mathrm{E}+1$ & $281 \pm 033 \mathrm{E}+1$ & $278 \pm 013 \mathrm{E}+1$ & $132 \pm 009 \mathrm{E}+1$ & $613 \pm 043 \mathrm{E}+0$ & $237 \pm 017 \mathrm{E}+0$ & $\ldots$ & $\ldots$ \\
\hline M81dwB & $114 \pm 012 \mathrm{E}-1$ & $903 \pm 116 \mathrm{E}-2$ & $270 \pm 018 \mathrm{E}-1$ & $161 \pm 027 \mathrm{E}-1$ & $292 \pm 018 \mathrm{E}-1$ & $176 \pm 014 \mathrm{E}-1$ & $104 \pm 010 \mathrm{E}-1$ & $426 \pm 213 \mathrm{E}-2$ & $\ldots$ & $\ldots$ \\
\hline NGC 3190 & $565 \pm 040 \mathrm{E}+0$ & $629 \pm 031 \mathrm{E}+0$ & $109 \pm 005 \mathrm{E}+1$ & $150 \pm 018 \mathrm{E}+1$ & $153 \pm 007 \mathrm{E}+1$ & $819 \pm 058 \mathrm{E}+0$ & $348 \pm 024 \mathrm{E}+0$ & $118 \pm 008 \mathrm{E}+0$ & $189 \pm 035 \mathrm{E}-1$ & $\ldots$ \\
\hline NGC 3184 & $157 \pm 011 \mathrm{E}+1$ & $180 \pm 009 \mathrm{E}+1$ & $392 \pm 019 \mathrm{E}+1$ & $704 \pm 084 \mathrm{E}+1$ & $537 \pm 026 \mathrm{E}+1$ & $314 \pm 022 \mathrm{E}+1$ & $146 \pm 010 \mathrm{E}+1$ & $577 \pm 041 \mathrm{E}+0$ & $\ldots$ & $126 \pm 009 \mathrm{E}+0$ \\
\hline NGC 3198 & $102 \pm 007 \mathrm{E}+1$ & $109 \pm 005 \mathrm{E}+1$ & $224 \pm 011 \mathrm{E}+1$ & $389 \pm 049 \mathrm{E}+1$ & $296 \pm 014 \mathrm{E}+1$ & $182 \pm 012 \mathrm{E}+1$ & $955 \pm 067 \mathrm{E}+0$ & $403 \pm 028 \mathrm{E}+0$ & $\ldots$ & $105 \pm 010 \mathrm{E}+0$ \\
\hline IC2574 & $483 \pm 034 \mathrm{E}+0$ & $578 \pm 029 \mathrm{E}+0$ & $937 \pm 047 \mathrm{E}+0$ & $105 \pm 012 \mathrm{E}+1$ & $986 \pm 049 \mathrm{E}+0$ & $569 \pm 040 \mathrm{E}+0$ & $412 \pm 029 \mathrm{E}+0$ & $165 \pm 012 \mathrm{E}+0$ & $\cdots$ & $\cdots$ \\
\hline NGC 3265 & $270 \pm 019 \mathrm{E}+0$ & $292 \pm 014 \mathrm{E}+0$ & $322 \pm 016 \mathrm{E}+0$ & $270 \pm 034 \mathrm{E}+0$ & $285 \pm 014 \mathrm{E}+0$ & $115 \pm 008 \mathrm{E}+0$ & $509 \pm 037 \mathrm{E}-1$ & $194 \pm 015 \mathrm{E}-1$ & $\cdots$ & $\cdots$ \\
\hline Mrk 33 & $434 \pm 031 \mathrm{E}+0$ & $\ldots$ & $\ldots$ & $386 \pm 047 \mathrm{E}+0$ & $\ldots$ & $\ldots$ & $\ldots$ & $\ldots$ & $400 \pm 060 \mathrm{E}-2$ & $\ldots$ \\
\hline NGC 3351 & $218 \pm 015 \mathrm{E}+1$ & $270 \pm 013 \mathrm{E}+1$ & $486 \pm 024 \mathrm{E}+1$ & $569 \pm 068 \mathrm{E}+1$ & $538 \pm 02$ & $3 \pm 022 \mathrm{E}+1$ & $138 \pm 00$ & $480 \pm 03$ & $\ldots$ & $102 \pm 00$ \\
\hline NGC 3521 & $644 \pm 045 \mathrm{E}+1$ & $800 \pm 040 \mathrm{E}+1$ & $163 \pm 008 \mathrm{E}+2$ & $195 \pm 023 \mathrm{E}+2$ & $203 \pm 010 \mathrm{E}+2$ & $106 \pm 007 \mathrm{E}+2$ & $459 \pm 032 \mathrm{E}+1$ & $164 \pm 011 \mathrm{E}+1$ & $210 \pm 082 \mathrm{E}+0$ & $312 \pm 014 \mathrm{E}+0$ \\
\hline NGC 3621 & $501 \pm 039 \mathrm{E}+1$ & $504 \pm 025 \mathrm{E}+1$ & $101 \pm 005 \mathrm{E}+2$ & $139 \pm 017 \mathrm{E}+2$ & $124 \pm 006 \mathrm{E}+2$ & $649 \pm 046 \mathrm{E}+1$ & $305 \pm 021 \mathrm{E}+1$ & $123 \pm 008 \mathrm{E}+1$ & $\ldots$ & $\ldots$ \\
\hline NGC 3627 & $918 \pm 070 \mathrm{E}+1$ & $107 \pm 005 \mathrm{E}+2$ & $188 \pm 009 \mathrm{E}+2$ & $215 \pm 027 \mathrm{E}+2$ & $196 \pm 009 \mathrm{E}+2$ & $904 \pm 064 \mathrm{E}+1$ & $360 \pm 025 \mathrm{E}+1$ & $120 \pm 008 \mathrm{E}+1$ & $186 \pm 070 \mathrm{E}+0$ & $228 \pm 011 \mathrm{E}+0$ \\
\hline NGC 3773 & $157 \pm 012 \mathrm{E}+0$ & $139 \pm 007 \mathrm{E}+0$ & $214 \pm 010 \mathrm{E}+0$ & $238 \pm 033 \mathrm{E}+0$ & $206 \pm 010 \mathrm{E}+0$ & $945 \pm 067 \mathrm{E}-1$ & $414 \pm 030 \mathrm{E}-1$ & $155 \pm 011 \mathrm{E}-1$ & $\ldots$ & $\ldots$ \\
\hline NGC 3938 & $142 \pm 010 \mathrm{E}+1$ & $164 \pm 008 \mathrm{E}+1$ & $307 \pm 015 \mathrm{E}+1$ & $519 \pm 062 \mathrm{E}+1$ & $392 \pm 019 \mathrm{E}+1$ & $219 \pm 015 \mathrm{E}+1$ & $983 \pm 069 \mathrm{E}+0$ & $365 \pm 026 \mathrm{E}+0$ & $\ldots$ & $883 \pm 083 \mathrm{E}-1$ \\
\hline NGC 4125 & $111 \pm 010 \mathrm{E}+0$ & $740 \pm 041 \mathrm{E}-1$ & $\ldots$ & $176 \pm 027 \mathrm{E}+0$ & $183 \pm 009 \mathrm{E}+0$ & $895 \pm 286 \mathrm{E}-1$ & $509 \pm 210 \mathrm{E}-1$ & $239 \pm 159 \mathrm{E}-1$ & $\ldots$ & $\ldots$ \\
\hline NGC 4236 & $801 \pm 056 \mathrm{E}+0$ & $803 \pm 041 \mathrm{E}+0$ & $113 \pm 005 \mathrm{E}+1$ & $161 \pm 019 \mathrm{E}+1$ & $172 \pm 008 \mathrm{E}+1$ & $107 \pm 007 \mathrm{E}+1$ & $694 \pm 049 \mathrm{E}+0$ & $356 \pm 025 \mathrm{E}+0$ & $\ldots$ & $727 \pm 101 \mathrm{E}-1$ \\
\hline NGC 4254 & $502 \pm 036 \mathrm{E}+1$ & $596 \pm 029 \mathrm{E}+1$ & $110 \pm 005 \mathrm{E}+2$ & $142 \pm 017 \mathrm{E}+2$ & $126 \pm 006 \mathrm{E}+2$ & $618 \pm 043 \mathrm{E}+1$ & $249 \pm 017 \mathrm{E}+1$ & $833 \pm 059 \mathrm{E}+0$ & $100 \pm 054 \mathrm{E}+0$ & $159 \pm 009 \mathrm{E}+0$ \\
\hline NGC 4321 & $405 \pm 028 \mathrm{E}+1$ & $440 \pm 022 \mathrm{E}+1$ & $907 \pm 045 \mathrm{E}+1$ & $139 \pm 016 \mathrm{E}+2$ & $116 \pm 005 \mathrm{E}+2$ & $627 \pm 044 \mathrm{E}+1$ & $265 \pm 018 \mathrm{E}+1$ & $901 \pm 064 \mathrm{E}+0$ & $875 \pm 493 \mathrm{E}-1$ & $165 \pm 008 \mathrm{E}+0$ \\
\hline NGC 4450 & $342 \pm 028 \mathrm{E}+0$ & $\cdots$ & $\cdots$ & $169 \pm 021 \mathrm{E}+1$ & $\cdots$ & $\cdots$ & $\cdots$ & $\cdots$ & $\cdots$ & $\cdots$ \\
\hline
\end{tabular}


Table 2

(Continued)

\begin{tabular}{|c|c|c|c|c|c|c|c|c|c|c|}
\hline Filter & $\begin{array}{l}\text { Spitzer } \\
\text { MIPS }\end{array}$ & $\begin{array}{c}\text { Herschel } \\
\text { PACS }\end{array}$ & $\begin{array}{c}\text { Herschel } \\
\text { PACS }\end{array}$ & $\begin{array}{l}\text { Spitzer } \\
\text { MIPS }\end{array}$ & $\begin{array}{c}\text { Herschel } \\
\text { PACS }\end{array}$ & $\begin{array}{l}\text { Herschel } \\
\text { SPIRE }\end{array}$ & $\begin{array}{l}\text { Herschel } \\
\text { SPIRE }\end{array}$ & $\begin{array}{l}\text { Herschel } \\
\text { SPIRE }\end{array}$ & $\begin{array}{c}\text { JCMT } \\
\text { SCUBA }\end{array}$ & $\begin{array}{c}\text { Planck } \\
\text { HFI }\end{array}$ \\
\hline $\bar{\lambda}(\mu \mathrm{m})$ & 72.5 & 71.8 & 103 & 157 & 167 & 252 & 353 & 511 & 850 & 850 \\
\hline$\Delta \lambda(\mu \mathrm{m})$ & 21.3 & 21.3 & 30.6 & 35.8 & 68.5 & 67.3 & 95.2 & 184 & 247 & 238 \\
\hline$A_{\lambda} / A_{V}$ & 0.00231 & 0.00231 & 0.00104 & 0.000388 & 0.000388 & 0.000152 & 0.0000725 & 0.0000361 & 0.0000146 & 0.0 \\
\hline NGC 4536 & $319 \pm 024 \mathrm{E}+1$ & $19 \pm 020 \mathrm{E}+1$ & $567 \pm 028 \mathrm{E}+1$ & $580 \pm 070 \mathrm{E}+1$ & $555 \pm 027 \mathrm{E}+1$ & $268 \pm 019 \mathrm{E}+1$ & $121 \pm 008 \mathrm{E}+1$ & $462 \pm 032 \mathrm{E}+0$ & $414 \pm 112 \mathrm{E}-1$ & $967 \pm 078 \mathrm{E}-1$ \\
\hline NGC 4552 & $537 \pm 110 \mathrm{E}-1$ & $\cdots$ & $\cdots$ & $142 \pm 073 \mathrm{E}+0$ & $\ldots$ & $\ldots$ & & & $\ldots$ & \\
\hline NGC 4559 & $168 \pm 011 \mathrm{E}+1$ & $180 \pm 009 \mathrm{E}+1$ & $337 \pm 016 \mathrm{E}+1$ & $541 \pm 065 \mathrm{E}+1$ & $405 \pm 020 \mathrm{E}+1$ & $236 \pm 016 \mathrm{E}+1$ & $122 \pm 008 \mathrm{E}+1$ & $529 \pm 037 \mathrm{E}+0$ & $\cdots$ & $148 \pm 011 \mathrm{E}+0$ \\
\hline NGC 4569 & $123 \pm 008 \mathrm{E}+1$ & $148 \pm 007 \mathrm{E}+1$ & $325 \pm 016 \mathrm{E}+1$ & $412 \pm 051 \mathrm{E}+1$ & $400 \pm 020 \mathrm{E}+1$ & $209 \pm 014 \mathrm{E}+1$ & $900 \pm 063 \mathrm{E}+0$ & $309 \pm 022 \mathrm{E}+0$ & $464 \pm 082 \mathrm{E}-1$ & $615 \pm 081 \mathrm{E}-1$ \\
\hline NGC 4579 & $952 \pm 075 \mathrm{E}+0$ & $999 \pm 050 \mathrm{E}+0$ & $254 \pm 012 \mathrm{E}+1$ & $410 \pm 049 \mathrm{E}+1$ & $345 \pm 017 \mathrm{E}+1$ & $194 \pm 013 \mathrm{E}+1$ & $844 \pm 059 \mathrm{E}+0$ & $297 \pm 021 \mathrm{E}+0$ & $439 \pm 066 \mathrm{E}-1$ & $625 \pm 112 \mathrm{E}-1$ \\
\hline NGC 4594 & $730 \pm 051 \mathrm{E}+0$ & $808 \pm 041 \mathrm{E}+0$ & $259 \pm 013 \mathrm{E}+1$ & $406 \pm 048 \mathrm{E}+1$ & $380 \pm 019 \mathrm{E}+1$ & $238 \pm 016 \mathrm{E}+1$ & $116 \pm 008 \mathrm{E}+1$ & $477 \pm 034 \mathrm{E}+0$ & $372 \pm 108 \mathrm{E}-1$ & $122 \pm 014 \mathrm{E}+0$ \\
\hline NGC 4625 & $185 \pm 013 \mathrm{E}+0$ & $151 \pm 008 \mathrm{E}+0$ & $376 \pm 019 \mathrm{E}+0$ & $508 \pm 061 \mathrm{E}+0$ & $469 \pm 023 \mathrm{E}+0$ & $256 \pm 018 \mathrm{E}+0$ & $130 \pm 009 \mathrm{E}+0$ & $549 \pm 040 \mathrm{E}-1$ & $\cdots$ & $\cdots$ \\
\hline NGC 4631 & $138 \pm 009 \mathrm{E}+2$ & $140 \pm 007 \mathrm{E}+2$ & $233 \pm 011 \mathrm{E}+2$ & $269 \pm 032 \mathrm{E}+2$ & $238 \pm 011 \mathrm{E}+2$ & $114 \pm 008 \mathrm{E}+2$ & $523 \pm 037 \mathrm{E}+1$ & $201 \pm 014 \mathrm{E}+1$ & $573 \pm 120 \mathrm{E}+0$ & $421 \pm 017 \mathrm{E}+0$ \\
\hline NGC 4725 & $885 \pm 066 \mathrm{E}+0$ & $103 \pm 005 E+1$ & $260 \pm 013 E+1$ & $599 \pm 073 \mathrm{E}+1$ & $466 \pm 023 \mathrm{E}+1$ & $302 \pm 021 \mathrm{E}+1$ & $160 \pm 011 \mathrm{E}+1$ & $648 \pm 046 \mathrm{E}+0$ & $\cdots$ & $128 \pm 011 \mathrm{E}+0$ \\
\hline NGC 4736 & $100 \pm 007 \mathrm{E}+2$ & $107 \pm 005 \mathrm{E}+2$ & $165 \pm 008 \mathrm{E}+2$ & $164 \pm 019 \mathrm{E}+2$ & $141 \pm 007 \mathrm{E}+2$ & $635 \pm 045 \mathrm{E}+1$ & $260 \pm 018 \mathrm{E}+1$ & $901 \pm 064 \mathrm{E}+0$ & $153 \pm 065 \mathrm{E}+0$ & $169 \pm 011 \mathrm{E}+0$ \\
\hline DDO 154 & $<591 \mathrm{E}-2$ & $<775 \mathrm{E}-2$ & $<912 \mathrm{E}-2$ & $<223 \mathrm{E}-1$ & $<801 \mathrm{E}-2$ & $<514 \mathrm{E}-2$ & $<483 \mathrm{E}-2$ & $<349 \mathrm{E}-2$ & $\cdots$ & $\cdots$ \\
\hline NGC 4826 & $528 \pm 037 \mathrm{E}+1$ & $563 \pm 028 \mathrm{E}+1$ & $975 \pm 048 \mathrm{E}+1$ & $857 \pm 102 \mathrm{E}+1$ & $906 \pm 045 \mathrm{E}+1$ & $393 \pm 027 \mathrm{E}+1$ & $156 \pm 011 \mathrm{E}+1$ & $516 \pm 036 \mathrm{E}+0$ & $123 \pm 030 \mathrm{E}+0$ & $101 \pm 008 \mathrm{E}+0$ \\
\hline DDO 165 & $<141 \mathrm{E}-1$ & $<976 \mathrm{E}-2$ & $<119 \mathrm{E}-1$ & $<205 \mathrm{E}-1$ & $<101 \mathrm{E}-1$ & $<762 \mathrm{E}-2$ & $<713 \mathrm{E}-2$ & $<484 \mathrm{E}-2$ & $\cdots$ & $\cdots$ \\
\hline NGC 5033 & $288 \pm 020 \mathrm{E}+1$ & $\cdots$ & $\cdots$ & $910 \pm 111 \mathrm{E}+1$ & $\cdots$ & $\ldots$ & $\ldots$ & $\ldots$ & $109 \pm 055 \mathrm{E}+0$ & $137 \pm 010 \mathrm{E}+0$ \\
\hline NGC 5055 & $744 \pm 052 \mathrm{E}+1$ & $764 \pm 038 \mathrm{E}+1$ & $175 \pm 008 \mathrm{E}+2$ & $273 \pm 032 \mathrm{E}+2$ & $241 \pm 012 \mathrm{E}+2$ & $138 \pm 009 \mathrm{E}+2$ & $611 \pm 043 \mathrm{E}+1$ & $221 \pm 015 \mathrm{E}+1$ & $\cdots$ & $397 \pm 015 \mathrm{E}+0$ \\
\hline NGC 5194 & $156 \pm 010 \mathrm{E}+2$ & $180 \pm 009 \mathrm{E}+2$ & $\cdots$ & $477 \pm 057 \mathrm{E}+2$ & $409 \pm 020 \mathrm{E}+2$ & $203 \pm 014 \mathrm{E}+2$ & $831 \pm 064 \mathrm{E}+1$ & $294 \pm 029 \mathrm{E}+1$ & $261 \pm 039 \mathrm{E}+0$ & $512 \pm 022 \mathrm{E}+0$ \\
\hline NGC 5195 & $971 \pm 068 \mathrm{E}+0$ & $217 \pm 010 \mathrm{E}+1$ & $\cdots$ & $129 \pm 015 \mathrm{E}+1$ & $279 \pm 013 \mathrm{E}+1$ & $23 \pm 009 \mathrm{E}+1$ & $446 \pm 040 \mathrm{E}+0$ & $152 \pm 022 \mathrm{E}+0$ & $259 \pm 039 \mathrm{E}-1$ & $\cdots$ \\
\hline NGC 5398 & $203 \pm 015 \mathrm{E}+0$ & $245 \pm 012 \mathrm{E}+0$ & $333 \pm 016 \mathrm{E}+0$ & $351 \pm 050 \mathrm{E}+0$ & $283 \pm 014 \mathrm{E}+0$ & $186 \pm 013 \mathrm{E}+0$ & $101 \pm 007 \mathrm{E}+0$ & $489 \pm 035 \mathrm{E}-1$ & $\cdots$ & $\cdots$ \\
\hline NGC 5457 & $118 \pm 008 \mathrm{E}+2$ & $135 \pm 006 \mathrm{E}+2$ & $262 \pm 013 \mathrm{E}+2$ & $399 \pm 047 \mathrm{E}+2$ & $336 \pm 016 \mathrm{E}+2$ & $194 \pm 013 \mathrm{E}+2$ & $942 \pm 066 \mathrm{E}+1$ & $388 \pm 027 \mathrm{E}+1$ & $\cdots$ & $\cdots$ \\
\hline NGC 5408 & $358 \pm 026 \mathrm{E}+0$ & $369 \pm 018 \mathrm{E}+0$ & $275 \pm 014 \mathrm{E}+0$ & $256 \pm 037 \mathrm{E}+0$ & $231 \pm 011 \mathrm{E}+0$ & $822 \pm 060 \mathrm{E}-1$ & $416 \pm 032 \mathrm{E}-1$ & $147 \pm 014 \mathrm{E}-1$ & $\cdots$ & $\cdots$ \\
\hline NGC 5474 & $347 \pm 024 \mathrm{E}+0$ & $354 \pm 018 \mathrm{E}+0$ & $604 \pm 031 \mathrm{E}+0$ & $913 \pm 109 \mathrm{E}+0$ & $853 \pm 043 \mathrm{E}+0$ & $482 \pm 034 \mathrm{E}+0$ & $280 \pm 020 \mathrm{E}+0$ & $134 \pm 009 \mathrm{E}+0$ & $\cdots$ & $377 \pm 085 \mathrm{E}-1$ \\
\hline NGC 5713 & $236 \pm 018 \mathrm{E}+1$ & $295 \pm 014 \mathrm{E}+1$ & $421 \pm 021 \mathrm{E}+1$ & $396 \pm 047 \mathrm{E}+1$ & $385 \pm 019 \mathrm{E}+1$ & $156 \pm 011 \mathrm{E}+1$ & $608 \pm 043 \mathrm{E}+0$ & $193 \pm 013 \mathrm{E}+0$ & $572 \pm 119 \mathrm{E}-1$ & $500 \pm 116 \mathrm{E}-1$ \\
\hline NGC 5866 & $870 \pm 063 \mathrm{E}+0$ & $880 \pm 044 \mathrm{E}+0$ & $180 \pm 009 \mathrm{E}+1$ & $177 \pm 021 \mathrm{E}+1$ & $178 \pm 008 \mathrm{E}+1$ & $757 \pm 053 \mathrm{E}+0$ & $305 \pm 021 \mathrm{E}+0$ & $965 \pm 072 \mathrm{E}-1$ & $140 \pm 020 \mathrm{E}-1$ & $192 \pm 067 \mathrm{E}-1$ \\
\hline IC4710 & $237 \pm 018 \mathrm{E}+0$ & $\cdots$ & $\cdots$ & $356 \pm 048 \mathrm{E}+0$ & $\cdots$ & $\cdots$ & $\cdots$ & $\cdots$ & $\cdots$ & $\cdots$ \\
\hline NGC 6822 & $636 \pm 044 \mathrm{E}+1$ & $\cdots$ & $\cdots$ & $143 \pm 017 \mathrm{E}+2$ & $\cdots$ & $\cdots$ & $\cdots$ & $\cdots$ & $\cdots$ & $\cdots$ \\
\hline NGC 6946 & $206 \pm 016 \mathrm{E}+2$ & $252 \pm 012 \mathrm{E}+2$ & $457 \pm 022 \mathrm{E}+2$ & $502 \pm 060 \mathrm{E}+2$ & $525 \pm 026 \mathrm{E}+2$ & $256 \pm 018 \mathrm{E}+2$ & $106 \pm 007 \mathrm{E}+2$ & $362 \pm 025 \mathrm{E}+1$ & $298 \pm 044 \mathrm{E}+0$ & $\cdots$ \\
\hline NGC 7331 & $749 \pm 066 \mathrm{E}+1$ & $688 \pm 034 \mathrm{E}+1$ & $135 \pm 006 \mathrm{E}+2$ & $189 \pm 024 \mathrm{E}+2$ & $171 \pm 008 \mathrm{E}+2$ & $882 \pm 062 \mathrm{E}+1$ & $385 \pm 027 \mathrm{E}+1$ & $139 \pm 009 \mathrm{E}+1$ & $211 \pm 038 \mathrm{E}+0$ & $\cdots$ \\
\hline NGC 7552 & $675 \pm 110 \mathrm{E}+1$ & $\cdots$ & $\cdots$ & $933 \pm 112 \mathrm{E}+1$ & $\cdots$ & $\cdots$ & $\cdots$ & $\cdots$ & $795 \pm 166 \mathrm{E}-1$ & $921 \pm 085 \mathrm{E}-1$ \\
\hline NGC 7793 & $329 \pm 023 \mathrm{E}+1$ & $350 \pm 017 \mathrm{E}+1$ & $695 \pm 034 \mathrm{E}+1$ & $107 \pm 012 \mathrm{E}+2$ & $901 \pm 045 \mathrm{E}+1$ & $526 \pm 037 \mathrm{E}+1$ & $276 \pm 019 \mathrm{E}+1$ & $117 \pm 008 \mathrm{E}+1$ & $\cdots$ & $283 \pm 013 \mathrm{E}+0$ \\
\hline
\end{tabular}

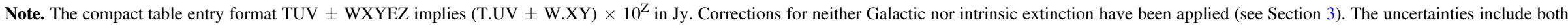
statistical and systematic effects. $5 \sigma$ upper limits are provided for non-detections. No color corrections have been applied. The filter central wavelengths and widths are computed via Equations (1) and (2).

${ }^{a}$ The Spitzer MIPS data for NGC 3034 suffer from saturation, and thus the tabulated values are lower limits. 
Table 3

$B V R_{\mathrm{C}} I_{\mathrm{C}}$ Photometry/Calibration Source

\begin{tabular}{|c|c|c|c|c|}
\hline Galaxy & $B$ & $V$ & $R_{\mathrm{C}}$ & $I_{\mathrm{C}}$ \\
\hline NGC 0024 & PS1 & PS1 & PS1 & PS1 \\
\hline NGC 0337 & PS1 & PS1 & PS1 & PS1 \\
\hline NGC 0584 & PS1 & PS1 & PS1 & PS1 \\
\hline NGC 0628 & PS1 & PS1 & PS1 & PS1 \\
\hline NGC 0855 & $\mathrm{RC} 3$ & $\mathrm{RC} 3$ & $\mathrm{C} 14$ & $\ldots$ \\
\hline NGC 0925 & PS1 & PS1 & PS1 & PS1 \\
\hline NGC 1097 & MM09 & MM09 & MM09 & MM09 \\
\hline NGC 1266 & PS1 & PS1 & PS1 & PS1 \\
\hline NGC 1291 & MM09 & MM09 & MM09 & MM09 \\
\hline NGC 1316 & MM09 & MM09 & MM09 & MM09 \\
\hline NGC 1377 & PS1 & PS1 & PS1 & PS1 \\
\hline NGC 1404 & MM09 & MM09 & MM09 & MM09 \\
\hline IC0342 & $\mathrm{RC} 3$ & $\ldots$ & $\ldots$ & $\ldots$ \\
\hline NGC 1482 & PS1 & PS1 & PS1 & PS1 \\
\hline NGC 1512 & MM09 & MM09 & MM09 & MM09 \\
\hline NGC 1566 & MM09 & MM09 & MM09 & MM09 \\
\hline NGC 1705 & MM09 & MM09 & MM09 & MM09 \\
\hline NGC 2403 & PS1 & PS1 & PS1 & PS1 \\
\hline NGC 2146 & $\mathrm{RC} 3$ & $\mathrm{RC} 3$ & $\ldots$ & T09 \\
\hline HolmbII & PS1 & PS1 & PS1 & PS1 \\
\hline M81dwA & PS1 & PS1 & PS1 & PS1 \\
\hline DDO 053 & PS1 & PS1 & PS1 & PS1 \\
\hline NGC 2798 & PS1 & PS1 & PS1 & PS1 \\
\hline NGC 2841 & PS1 & PS1 & PS1 & PS1 \\
\hline NGC 2915 & $\mathrm{RC} 3$ & MM09 & MM09 & D07 \\
\hline HolmbI & PS1 & PS1 & PS1 & PS1 \\
\hline NGC 2976 & PS1 & PS1 & PS1 & PS1 \\
\hline NGC 3049 & PS1 & PS1 & PS1 & PS1 \\
\hline NGC 3031 & $\mathrm{RC} 3$ & $\mathrm{RC} 3$ & $\ldots$ & T09 \\
\hline NGC 3034 & PS1 & PS1 & PS1 & PS1 \\
\hline HolmbIX & PS1 & PS1 & PS1 & PS1 \\
\hline NGC 3077 & $\mathrm{C} 14$ & $\ldots$ & $\mathrm{RC} 3$ & $\ldots$ \\
\hline M81dwB & PS1 & PS1 & PS1 & PS1 \\
\hline NGC 3190 & PS1 & PS1 & PS1 & PS1 \\
\hline NGC 3184 & PS1 & PS1 & PS1 & PS1 \\
\hline NGC 3198 & PS1 & PS1 & PS1 & PS1 \\
\hline IC2574 & PS1 & PS1 & PS1 & PS1 \\
\hline NGC 3265 & PS1 & PS1 & PS1 & PS1 \\
\hline Mrk 33 & PS1 & PS1 & PS1 & PS1 \\
\hline NGC 3351 & PS1 & PS1 & PS1 & PS1 \\
\hline NGC 3521 & PS1 & PS1 & PS1 & PS1 \\
\hline NGC 3621 & MM09 & MM09 & MM09 & MM09 \\
\hline NGC 3627 & PS1 & PS1 & PS1 & PS1 \\
\hline NGC 3773 & PS1 & PS1 & PS1 & PS1 \\
\hline NGC 3938 & PS1 & PS1 & PS1 & PS1 \\
\hline NGC 4125 & PS1 & PS1 & PS1 & PS1 \\
\hline NGC 4236 & PS1 & PS1 & PS1 & PS1 \\
\hline NGC 4254 & PS1 & PS1 & PS1 & PS1 \\
\hline NGC 4321 & PS1 & PS1 & PS1 & PS1 \\
\hline NGC 4450 & PS1 & PS1 & PS1 & PS1 \\
\hline NGC 4536 & PS1 & PS1 & PS1 & PS1 \\
\hline NGC 4552 & PS1 & PS1 & PS1 & PS1 \\
\hline NGC 4559 & PS1 & PS1 & PS1 & PS1 \\
\hline NGC 4569 & $\mathrm{RC} 3$ & $\mathrm{RC} 3$ & $\ldots$ & $\ldots$ \\
\hline NGC 4579 & PS1 & PS1 & PS1 & PS1 \\
\hline NGC 4594 & PS1 & PS1 & PS1 & PS1 \\
\hline NGC 4625 & PS1 & PS1 & PS1 & PS1 \\
\hline NGC 4631 & PS1 & PS1 & PS1 & PS1 \\
\hline NGC 4725 & PS1 & PS1 & PS1 & PS1 \\
\hline NGC 4736 & PS1 & PS1 & PS1 & PS1 \\
\hline DDO 154 & PS1 & PS1 & PS1 & PS1 \\
\hline NGC 4826 & PS1 & PS1 & PS1 & PS1 \\
\hline DDO 165 & PS1 & PS1 & PS1 & PS1 \\
\hline NGC 5033 & PS1 & PS1 & $\ldots$ & PS1 \\
\hline
\end{tabular}

Table 3

(Continued)

\begin{tabular}{lllll}
\hline \hline Galaxy & $B$ & $V$ & $R_{\mathrm{C}}$ & $I_{\mathrm{C}}$ \\
\hline NGC 5055 & RC3 & RC3 & C14 & T09 \\
NGC 5194 & PS1 & PS1 & PS1 & PS1 \\
NGC 5195 & PS1 & PS1 & PS1 & PS1 \\
NGC 5398 & D07 & D07 & D07 & D07 \\
NGC 5457 & RC3 & RC3 & C14 & T09 \\
NGC 5408 & RC3 & RC3 & $\ldots$ & $\ldots$ \\
NGC 5474 & PS1 & PS1 & PS1 & PS1 \\
NGC 5713 & PS1 & PS1 & PS1 & PS1 \\
NGC 5866 & PS1 & PS1 & PS1 & PS1 \\
IC4710 & D07 & D07 & D07 & $\ldots$ \\
NGC 6822 & PS1 & PS1 & PS1 & PS1 \\
NGC 6946 & RC3 & PS1 & $\ldots$ & PS1 \\
NGC 7331 & PS1 & PS1 & PS1 & PS1 \\
NGC 7552 & MM09 & MM09 & D07 & D07 \\
NGC 7793 & D07 & D07 & D07 & D07 \\
\hline
\end{tabular}

Note. PS1: recalibration of Dale et al. (2007) photometry using Pan-STARRS1 (Section 3.1); D07: Dale et al. (2007); MM09: Muñoz-Mateos et al. (2009); C14: Cook et al. (2014); T09: unpublished photometry from M. Pierce via the Extragalactic Distance Database (Tully et al. 2009); RC3: de Vaucouleurs et al. (1991).

procedures for these 6 galaxies are described in Bendo et al. (2010).

The Herschel PACS and SPIRE imaging data for these $61+6=67$ KINGFISH + VNGS galaxies for this publication were processed from Level 0 to Level 1 using HIPE Version 11.1.0, ${ }^{24}$ and the Level 1 to Level 2 post-pipeline processing used Scanamorphos Version 24.0 (Roussel 2013); the data published in Dale et al. (2012) were processed using HIPE Version 5.0 and Scanamorphos Version 12.5. With this newer version of Scanamorphos, the PACS distortion flatfield is now properly incorporated. In practice, this has decreased the noise levels in the PACS maps and slightly modified the PACS flux calibration $(\sim 1 \%)$. Moreover, the destriping of PACS observations of large and diffuse fields is substantially improved, and the subtraction of the average drift on short timescales no longer introduces low-level noise. These changes allow for more secure detections of diffuse emission and more robust estimates of sky levels.

One important factor involved in the SPIRE flux calibration is the SPIRE beam size, since the SPIRE images are converted into surface brightness units by dividing by the estimated beam areas. The updated beam sizes used for this work at [250, 350, 500] $\mu \mathrm{m}$ are $[469.35,831.27,1804.31] \operatorname{~arcsec}^{2}$, representing percentage increases of $[11.0,10.7,13.7] \%$ compared to the previous values of $[423,751,1587] \operatorname{arcsec}^{2}$ used by Dale et al. (2012). These updated values are the recommended beam sizes in Version 3.0 (2016 June 03) of the SPIRE Handbook.

The PACS fractional calibration uncertainties are of order $\epsilon_{\text {cal }} /$ $f_{\nu} \sim 5 \%$, according to Version 2.5.1 (2013 July 09) of the PACS Observer's Manual. Calibration uncertainties for SPIRE data are estimated at $\epsilon_{\mathrm{cal}} / f_{\nu} \sim 7 \%$, also taken from Version 3.0 of the SPIRE Observer's Manual. This level of uncertainty in the SPIRE calibration is a sum in quadrature of the uncertainties in the absolute and relative calibrations $(\sim 5.5 \%)$ along with the uncertainties in the extended source calibration $(\sim 4 \%)$.

\footnotetext{
24 Version 14.0.0 was used for the six VNGS galaxies. This version provides better deglitched SPIRE maps and a slightly improved extended source calibration for PACS.
} 


\subsection{WISE $12 \mu \mathrm{m}$}

WISE $12 \mu \mathrm{m}$ imaging (Wright et al. 2010) is used here to help bridge the gap in wavelength coverage in our broadband SEDs between the Spitzer 8 and $24 \mu \mathrm{m}$ bandpasses. The $12 \mu \mathrm{m}$ bandpass is an important tracer of the PAH complexes centered near (restframe) wavelengths of 11.3, 12.7, and $17 \mu \mathrm{m}$ (Smith et al. 2007). At $12 \mu \mathrm{m}$, the (single-frame) WISE PSF full-width at half maximum (FWHM) is 6!"5 and the photometric calibration $\sim 7 \% .^{25}$ A full suite of WISE W1, W2, W3, and W4 $(3.4,4.6,12$, and $22 \mu \mathrm{m})$ "total" flux densities is provided in Table 4 (see the Appendix). We use native resolution imaging (not the drizzled Atlas release imaging), which provides superior resolution and minimizes the need for aperture corrections.

\subsection{Planck $850 \mu \mathrm{m}$}

There are SCUBA $850 \mu \mathrm{m}$ flux densities available for only 27 galaxies in the SINGS/KINGFISH sample, and we therefore supplement these data with Planck $850 \mu \mathrm{m}$ flux densities for 36 galaxies; the total number of $850 \mu \mathrm{m}$ detections for the combined Planck/SCUBA SINGS/KINGFISH sample is 43. We use cataloged Planck $850 \mu \mathrm{m}$ flux densities derived from the APERFLUX technique, a technique that employs circular apertures plus concentric sky annuli. Of the different flavors of Planck flux density extractions, APERFLUX is the simplest method and relies on the fewest assumptions; the Planck team recommends APERFLUX for wavelengths $850 \mu \mathrm{m}$ and shorter (Planck Collaboration et al. 2016a). Although some of the SCUBA $850 \mu \mathrm{m}$ data suffer from relatively small maps and spatial filtering issues, the lower resolution Planck data may be affected by foreground or background sources.

\subsection{Sky Estimation and Elimination of Spatial Interlopers}

The "sky" in the direction of any galaxy is a superposition of emission from faint foreground stars and background galaxies, interstellar emission, and in the case of ground-based observations, the Earth's atmosphere. To determine the sky level for each image, a set of sky apertures has been defined (by eye) that collectively circumscribe the galaxy, projected on the sky close enough to the galaxy to measure the "local" sky, but far enough away to avoid any galaxy emission (the process is unchanged from Dale et al. 2012, see their Figure 1).

Before estimating the sky levels and executing aperture photometry, any emission from obvious foreground stars or from neighboring or background galaxies is identified and removed from the areas covered by each galaxy's aperture and collection of sky apertures. The identification is assisted by ancillary data at shorter wavelengths and higher spatial resolution (e.g., Spitzer/IRAC 3.6 and $8.0 \mu \mathrm{m}, H S T$ optical, and ground-based $\mathrm{H} \alpha$ imaging). The removal is accomplished via IRAF/IMEDIT by replacing the values of contaminated pixels via a $2 \mathrm{D}$ surface fit to to a nearby sky annulus of width 2 pixels, with noise added that matches the noise statistics of the sky annulus. These annuli only sample the local sky around each spatial interloper and thus do not capture the full sky variations across the image. Fortunately, the spatial interlopers

\footnotetext{
25 Explanatory Supplement to the WISE All-Sky Data Release Products; 2015 February 19.
}

are much smaller than our target galaxies, a fact that limits the effect of any shortcomings in the contaminant-removal procedure.

The total sky area, derived from the sum of the areas from all sky apertures, is typically significantly greater than the area covered by the galaxy aperture itself, thereby limiting the contribution of uncertainty in the sky level to the overall error budget. The mean sky level per pixel is computed from the collection of these sky apertures, the value is scaled to the number of pixels in the galaxy aperture, and the result is subtracted from the overall galaxy aperture counts.

\subsection{Aperture Photometry}

The elliptical apertures used for global photometry are listed in Table 1, and the same aperture is used to extract the flux at each wavelength. The apertures were chosen to encompass essentially all of the detectable emission at every wavelength (see also Dale et al. 2005, 2007, 2012). The average ratio of aperture major axis length $2 a$ to the de Vaucouleurs $D_{25}$ optical major axis is 1.45 (with a $1 \sigma$ dispersion in this ratio of 0.45 ).

At the longest wavelengths where the imaging resolution is typically coarsest, a small portion of the galaxy emission may appear beyond the chosen apertures. Thus, for the Spitzer and Herschel photometry we use the aperture corrections described in Dale et al. (2007, 2012). No aperture corrections were applied to the WISE photometry as they are negligible for the large apertures used here on native resolution imaging.

The uncertainties in the integrated photometry $\epsilon_{\text {total }}$ are computed as a combination in quadrature of the calibration uncertainty $\epsilon_{\mathrm{cal}}$ and the measurement uncertainty $\epsilon_{\mathrm{sky}}$ based on the measured sky fluctuations and the areas covered by the galaxy and the sum of the sky apertures, i.e.,

$$
\epsilon_{\mathrm{total}}=\sqrt{\epsilon_{\mathrm{cal}}^{2}+\epsilon_{\mathrm{sky}}^{2}}
$$

with

$$
\epsilon_{\text {sky }}=\sigma_{\text {sky }} \Omega_{\text {pix }} \sqrt{N_{\text {pix }}+N_{\text {pix }}^{2} / N_{\text {sky }}}
$$

where $\sigma_{\text {sky }}$ is the standard deviation of the sky surface brightness fluctuations, $\Omega_{\text {pix }}$ is the solid angle subtended per pixel, and $N_{\text {pix }}$ and $N_{\text {sky }}$ are the number of pixels in the galaxy and (the sum of) the sky apertures, respectively. For the few sources undetected by Spitzer, Herschel, or WISE imaging, $5 \sigma$ upper limits are derived assuming a galaxy spans all $N_{\text {pix }}$ pixels in the aperture,

$$
f_{\nu}(5 \sigma \text { upper limit })=5 \epsilon_{\mathrm{sky}}
$$

Based on our visual scrutiny of the imaging data sets from a given telescope, any images redward of a non-detection are also considered to yield non-detections.

The galaxy apertures, the sky apertures, and the foreground stellar masks are provided with the electronic version of the journal article. 
Table 4

WISE and VLA $20 \mathrm{~cm}$ Total Flux Densities in Janskys Corrected for neither Galactic nor Intrinsic Extinction

\begin{tabular}{|c|c|c|c|c|c|}
\hline & $\begin{array}{c}\text { WISE } \\
\mathrm{W} 1\end{array}$ & WISE & WISE & WISE & NRAO \\
\hline Filter & W1 & W2 & W3 & W4 & VLA \\
\hline $\bar{\lambda}(\mu \mathrm{m})$ & 3.40 & 4.65 & 12.8 & 22.4 & $20 \mathrm{~cm}$ \\
\hline$\Delta \lambda(\mu \mathrm{m})$ & 0.663 & 1.04 & 5.51 & 4.11 & \\
\hline$A_{\lambda} / A_{V}$ & 0.0523 & 0.0287 & 0.0285 & 0.0225 & 0.0 \\
\hline NGC0024 & $108 \pm 007 \mathrm{E}-1$ & $544 \pm 038 \mathrm{E}-2$ & $895 \pm 068 \mathrm{E}-2$ & $109 \pm 009 \mathrm{E}-1$ & $\cdots$ \\
\hline NGC0337 & $981 \pm 069 \mathrm{E}-2$ & $693 \pm 050 \mathrm{E}-2$ & $310 \pm 022 \mathrm{E}-1$ & $733 \pm 053 \mathrm{E}-1$ & $109 \pm 010 \mathrm{E}-1$ \\
\hline NGC0584 & $420 \pm 029 \mathrm{E}-1$ & $208 \pm 014 \mathrm{E}-1$ & $661 \pm 055 \mathrm{E}-2$ & $335 \pm 040 \mathrm{E}-2$ & $<500 \mathrm{E}-2$ \\
\hline NGC0628 & $842 \pm 059 \mathrm{E}-1$ & $513 \pm 036 \mathrm{E}-1$ & $233 \pm 016 \mathrm{E}+0$ & $329 \pm 023 \mathrm{E}+0$ & $172 \pm 017 \mathrm{E}-1$ \\
\hline NGC0855 & $442 \pm 031 \mathrm{E}-2$ & $243 \pm 018 \mathrm{E}-2$ & $391 \pm 034 \mathrm{E}-2$ & $710 \pm 056 \mathrm{E}-2$ & $490 \pm 050 \mathrm{E}-3$ \\
\hline NGC0925 & $304 \pm 021 \mathrm{E}-1$ & $177 \pm 013 \mathrm{E}-1$ & $535 \pm 040 \mathrm{E}-1$ & $864 \pm 076 \mathrm{E}-1$ & $460 \pm 050 \mathrm{E}-2$ \\
\hline NGC1097 & $126 \pm 008 \mathrm{E}+0$ & $765 \pm 054 \mathrm{E}-1$ & $293 \pm 020 \mathrm{E}+0$ & $685 \pm 048 \mathrm{E}+0$ & $414 \pm 041 \mathrm{E}-1$ \\
\hline NGC1266 & $559 \pm 039 \mathrm{E}-2$ & $399 \pm 028 \mathrm{E}-2$ & $932 \pm 067 \mathrm{E}-2$ & $740 \pm 053 \mathrm{E}-1$ & $116 \pm 012 \mathrm{E}-1$ \\
\hline NGC1291 & $226 \pm 016 \mathrm{E}+0$ & $119 \pm 008 \mathrm{E}+0$ & $563 \pm 050 \mathrm{E}-1$ & $594 \pm 069 \mathrm{E}-1$ & $\ldots$ \\
\hline NGC1316 & $293 \pm 020 \mathrm{E}+0$ & $151 \pm 010 \mathrm{E}+0$ & $527 \pm 039 \mathrm{E}-1$ & $419 \pm 034 \mathrm{E}-1$ & $255 \pm 026 \mathrm{E}-1$ \\
\hline NGC1377 & $503 \pm 035 E-2$ & $948 \pm 067 \mathrm{E}-2$ & $390 \pm 027 \mathrm{E}-1$ & $163 \pm 011 \mathrm{E}+0$ & $<100 \mathrm{E}-3$ \\
\hline NGC1404 & $721 \pm 051 \mathrm{E}-1$ & $370 \pm 026 \mathrm{E}-1$ & $166 \pm 017 \mathrm{E}-1$ & $740 \pm 073 \mathrm{E}-2$ & $390 \pm 060 \mathrm{E}-3$ \\
\hline IC0342 & $828 \pm 058 \mathrm{E}+0$ & $486 \pm 034 \mathrm{E}+0$ & $237 \pm 018 \mathrm{E}+1$ & $418 \pm 029 \mathrm{E}+1$ & $240 \pm 024 \mathrm{E}+0$ \\
\hline NGC1482 & $205 \pm 014 \mathrm{E}-1$ & $150 \pm 010 \mathrm{E}-1$ & $119 \pm 008 \mathrm{E}+0$ & $353 \pm 025 \mathrm{E}+0$ & $238 \pm 024 \mathrm{E}-1$ \\
\hline NGC1512 & $400 \pm 028 \mathrm{E}-1$ & $208 \pm 014 \mathrm{E}-1$ & $386 \pm 028 \mathrm{E}-1$ & $468 \pm 035 \mathrm{E}-1$ & $700 \pm 100 \mathrm{E}-3$ \\
\hline NGC1566 & $740 \pm 052 \mathrm{E}-1$ & $446 \pm 031 \mathrm{E}-1$ & $188 \pm 013 \mathrm{E}+0$ & $292 \pm 020 \mathrm{E}+0$ & $400 \pm 000 \mathrm{E}-1$ \\
\hline NGC1705 & $301 \pm 021 \mathrm{E}-2$ & $162 \pm 011 \mathrm{E}-2$ & $193 \pm 015 \mathrm{E}-2$ & $562 \pm 052 \mathrm{E}-2$ & $\cdots$ \\
\hline NGC2146 & $873 \pm 061 \mathrm{E}-1$ & $659 \pm 046 \mathrm{E}-1$ & $546 \pm 038 \mathrm{E}+0$ & $160 \pm 011 \mathrm{E}+1$ & $107 \pm 010 \mathrm{E}+0$ \\
\hline NGC2403 & $169 \pm 011 \mathrm{E}+0$ & $101 \pm 007 \mathrm{E}+0$ & $365 \pm 026 \mathrm{E}+0$ & $614 \pm 045 \mathrm{E}+0$ & $330 \pm 033 \mathrm{E}-1$ \\
\hline HoII & $\cdots$ & $\cdots$ & $\cdots$ & $\cdots$ & $197 \pm 028 \mathrm{E}-2$ \\
\hline M81dwA & $080 \pm 010 \mathrm{E}-4$ & $\ldots$ & $\ldots$ & $\ldots$ & $\ldots$ \\
\hline DDO053 & $460 \pm 045 \mathrm{E}-3$ & $297 \pm 037 \mathrm{E}-3$ & $\ldots$ & $338 \pm 047 \mathrm{E}-2$ & $\ldots$ \\
\hline NGC2798 & $107 \pm 007 \mathrm{E}-1$ & $762 \pm 054 \mathrm{E}-2$ & $564 \pm 040 \mathrm{E}-1$ & $238 \pm 016 \mathrm{E}+0$ & $829 \pm 085 \mathrm{E}-2$ \\
\hline NGC2841 & $139 \pm 009 \mathrm{E}+0$ & $759 \pm 054 \mathrm{E}-1$ & $895 \pm 063 \mathrm{E}-1$ & $112 \pm 008 \mathrm{E}+0$ & $840 \pm 086 \mathrm{E}-2$ \\
\hline NGC2915 & $537 \pm 038 \mathrm{E}-2$ & $296 \pm 021 \mathrm{E}-2$ & $218 \pm 017 \mathrm{E}-2$ & $513 \pm 040 \mathrm{E}-2$ & $\ldots$ \\
\hline HoI & $\ldots$ & $\ldots$ & $\ldots$ & $\ldots$ & $\cdots$ \\
\hline NGC2976 & $433 \pm 030 \mathrm{E}-1$ & $255 \pm 018 \mathrm{E}-1$ & $854 \pm 061 \mathrm{E}-1$ & $140 \pm 010 \mathrm{E}+0$ & $508 \pm 054 \mathrm{E}-2$ \\
\hline NGC3049 & $399 \pm 028 \mathrm{E}-2$ & $223 \pm 016 \mathrm{E}-2$ & $107 \pm 007 \mathrm{E}-1$ & $385 \pm 028 \mathrm{E}-1$ & $120 \pm 023 \mathrm{E}-2$ \\
\hline NGC3031 & $112 \pm 007 \mathrm{E}+1$ & $618 \pm 043 \mathrm{E}+0$ & $527 \pm 037 \mathrm{E}+0$ & $490 \pm 035 \mathrm{E}+0$ & $379 \pm 037 \mathrm{E}-1$ \\
\hline NGC3034 & $733 \pm 051 \mathrm{E}+0$ & $614 \pm 043 \mathrm{E}+0$ & $713 \pm 050 \mathrm{E}+1$ & $356 \pm 025 \mathrm{E}+2$ & $765 \pm 077 \mathrm{E}+0$ \\
\hline HoIX & $\cdots$ & $\cdots$ & $\cdots$ & $\cdots$ & $\cdots$ \\
\hline NGC3077 & $615 \pm 043 \mathrm{E}-1$ & $356 \pm 025 \mathrm{E}-1$ & $732 \pm 053 \mathrm{E}-1$ & $182 \pm 013 \mathrm{E}+0$ & $\cdots$ \\
\hline M81dwB & $559 \pm 043 \mathrm{E}-3$ & $327 \pm 029 \mathrm{E}-3$ & $\ldots$ & $\ldots$ & $\ldots$ \\
\hline NGC3190 & $377 \pm 026 \mathrm{E}-1$ & $214 \pm 015 \mathrm{E}-1$ & $267 \pm 019 \mathrm{E}-1$ & $259 \pm 019 \mathrm{E}-1$ & $430 \pm 047 \mathrm{E}-2$ \\
\hline NGC3184 & $504 \pm 035 \mathrm{E}-1$ & $290 \pm 020 \mathrm{E}-1$ & $114 \pm 008 \mathrm{E}+0$ & $146 \pm 010 \mathrm{E}+0$ & $559 \pm 059 \mathrm{E}-2$ \\
\hline NGC3198 & $288 \pm 020 \mathrm{E}-1$ & $171 \pm 012 \mathrm{E}-1$ & $596 \pm 043 \mathrm{E}-1$ & $105 \pm 007 \mathrm{E}+0$ & $270 \pm 034 \mathrm{E}-2$ \\
\hline IC2574 & $121 \pm 009 \mathrm{E}-1$ & $688 \pm 059 \mathrm{E}-2$ & $354 \pm 037 \mathrm{E}-2$ & $\ldots$ & $107 \pm 023 \mathrm{E}-2$ \\
\hline NGC3265 & $262 \pm 018 \mathrm{E}-2$ & $156 \pm 011 \mathrm{E}-2$ & $786 \pm 056 \mathrm{E}-2$ & $261 \pm 018 \mathrm{E}-1$ & $111 \pm 023 \mathrm{E}-2$ \\
\hline Mrk33 & $259 \pm 018 \mathrm{E}-2$ & $173 \pm 012 \mathrm{E}-2$ & $144 \pm 010 \mathrm{E}-1$ & $788 \pm 056 \mathrm{E}-1$ & $172 \pm 026 \mathrm{E}-2$ \\
\hline NGC3351 & $813 \pm 057 \mathrm{E}-1$ & $433 \pm 030 \mathrm{E}-1$ & $114 \pm 008 \mathrm{E}+0$ & $246 \pm 017 \mathrm{E}+0$ & $438 \pm 048 \mathrm{E}-2$ \\
\hline NGC3521 & $208 \pm 014 \mathrm{E}+0$ & $121 \pm 008 \mathrm{E}+0$ & $486 \pm 034 \mathrm{E}+0$ & $597 \pm 042 \mathrm{E}+0$ & $356 \pm 035 \mathrm{E}-1$ \\
\hline NGC3621 & $889 \pm 064 \mathrm{E}-1$ & $612 \pm 045 \mathrm{E}-1$ & $276 \pm 019 \mathrm{E}+0$ & $366 \pm 026 \mathrm{E}+0$ & $197 \pm 019 \mathrm{E}-1$ \\
\hline NGC3627 & $187 \pm 013 \mathrm{E}+0$ & $112 \pm 008 \mathrm{E}+0$ & $458 \pm 032 \mathrm{E}+0$ & $788 \pm 056 \mathrm{E}+0$ & $457 \pm 045 \mathrm{E}-1$ \\
\hline NGC3773 & $210 \pm 015 \mathrm{E}-2$ & $121 \pm 008 \mathrm{E}-2$ & $399 \pm 030 \mathrm{E}-2$ & $124 \pm 009 \mathrm{E}-1$ & $580 \pm 050 \mathrm{E}-3$ \\
\hline NGC3938 & $315 \pm 022 \mathrm{E}-1$ & $197 \pm 014 \mathrm{E}-1$ & $848 \pm 060 \mathrm{E}-1$ & $112 \pm 008 \mathrm{E}+0$ & $617 \pm 065 \mathrm{E}-2$ \\
\hline NGC4125 & $794 \pm 056 \mathrm{E}-1$ & $399 \pm 028 \mathrm{E}-1$ & $120 \pm 010 \mathrm{E}-1$ & $670 \pm 062 \mathrm{E}-2$ & $<500 \mathrm{E}-2$ \\
\hline NGC4236 & $229 \pm 016 \mathrm{E}-1$ & $124 \pm 010 \mathrm{E}-1$ & $134 \pm 014 \mathrm{E}-1$ & $\ldots$ & $281 \pm 034 \mathrm{E}-2$ \\
\hline NGC4254 & $683 \pm 048 \mathrm{E}-1$ & $445 \pm 031 \mathrm{E}-1$ & $332 \pm 023 \mathrm{E}+0$ & $448 \pm 031 \mathrm{E}+0$ & $421 \pm 041 \mathrm{E}-1$ \\
\hline NGC4321 & $100 \pm 007 \mathrm{E}+0$ & $625 \pm 045 \mathrm{E}-1$ & $258 \pm 018 \mathrm{E}+0$ & $378 \pm 027 \mathrm{E}+0$ & $340 \pm 034 \mathrm{E}-1$ \\
\hline NGC4450 & $552 \pm 039 \mathrm{E}-1$ & $297 \pm 021 \mathrm{E}-1$ & $221 \pm 016 \mathrm{E}-1$ & $223 \pm 020 \mathrm{E}-1$ & $940 \pm 100 \mathrm{E}-3$ \\
\hline NGC4536 & $422 \pm 029 \mathrm{E}-1$ & $267 \pm 019 \mathrm{E}-1$ & $132 \pm 009 \mathrm{E}+0$ & $349 \pm 025 \mathrm{E}+0$ & $194 \pm 018 \mathrm{E}-1$ \\
\hline NGC4552 & $918 \pm 065 \mathrm{E}-1$ & $447 \pm 036 \mathrm{E}-1$ & $256 \pm 034 \mathrm{E}-1$ & $999 \pm 138 \mathrm{E}-2$ & $100 \pm 003 \mathrm{E}-1$ \\
\hline NGC4559 & $352 \pm 024 \mathrm{E}-1$ & $230 \pm 016 \mathrm{E}-1$ & $677 \pm 048 \mathrm{E}-1$ & $114 \pm 008 \mathrm{E}+0$ & $654 \pm 068 \mathrm{E}-2$ \\
\hline NGC4569 & $793 \pm 056 \mathrm{E}-1$ & $448 \pm 031 \mathrm{E}-1$ & $894 \pm 063 \mathrm{E}-1$ & $139 \pm 009 \mathrm{E}+0$ & $834 \pm 086 \mathrm{E}-2$ \\
\hline NGC4579 & $929 \pm 065 \mathrm{E}-1$ & $498 \pm 035 \mathrm{E}-1$ & $610 \pm 043 \mathrm{E}-1$ & $687 \pm 049 \mathrm{E}-1$ & $984 \pm 100 \mathrm{E}-2$ \\
\hline NGC4594 & $444 \pm 031 \mathrm{E}+0$ & $236 \pm 016 \mathrm{E}+0$ & $113 \pm 008 \mathrm{E}+0$ & $893 \pm 070 \mathrm{E}-1$ & $136 \pm 014 \mathrm{E}-1$ \\
\hline NGC4625 & $477 \pm 033 \mathrm{E}-2$ & $275 \pm 019 \mathrm{E}-2$ & $100 \pm 007 \mathrm{E}-1$ & $127 \pm 011 \mathrm{E}-1$ & $710 \pm 210 \mathrm{E}-3$ \\
\hline NGC4631 & $124 \pm 008 \mathrm{E}+0$ & $804 \pm 056 \mathrm{E}-1$ & $469 \pm 033 \mathrm{E}+0$ & $839 \pm 059 \mathrm{E}+0$ & $120 \pm 012 \mathrm{E}+0$ \\
\hline NGC4725 & $116 \pm 008 \mathrm{E}+0$ & $598 \pm 042 \mathrm{E}-1$ & $740 \pm 053 \mathrm{E}-1$ & $721 \pm 053 \mathrm{E}-1$ & $280 \pm 034 \mathrm{E}-2$ \\
\hline NGC4736 & $389 \pm 027 \mathrm{E}+0$ & $221 \pm 015 \mathrm{E}+0$ & $447 \pm 031 \mathrm{E}+0$ & $586 \pm 042 \mathrm{E}+0$ & $270 \pm 027 \mathrm{E}-1$ \\
\hline DDO154 & $443 \pm 038 \mathrm{E}-3$ & & $\ldots$ & $\ldots$ & \\
\hline
\end{tabular}


Table 4

(Continued)

\begin{tabular}{|c|c|c|c|c|c|}
\hline & WISE & WISE & WISE & WISE & NRAO \\
\hline Filter & W1 & W2 & W3 & W4 & VLA \\
\hline $\bar{\lambda}(\mu \mathrm{m})$ & 3.40 & 4.65 & 12.8 & 22.4 & $20 \mathrm{~cm}$ \\
\hline$\Delta \lambda(\mu \mathrm{m})$ & 0.663 & 1.04 & 5.51 & 4.11 & \\
\hline$A_{\lambda} / A_{V}$ & 0.0523 & 0.0287 & 0.0285 & 0.0225 & 0.0 \\
\hline NGC4826 & $265 \pm 018 \mathrm{E}+0$ & $145 \pm 010 \mathrm{E}+0$ & $197 \pm 014 \mathrm{E}+0$ & $265 \pm 019 \mathrm{E}+0$ & $101 \pm 009 \mathrm{E}-1$ \\
\hline DDO165 & $124 \pm 009 \mathrm{E}-2$ & $\ldots$ & $\ldots$ & $\ldots$ & $\ldots$ \\
\hline NGC5033 & $642 \pm 045 \mathrm{E}-1$ & $389 \pm 027 \mathrm{E}-1$ & $169 \pm 012 \mathrm{E}+0$ & $210 \pm 015 \mathrm{E}+0$ & $178 \pm 017 \mathrm{E}-1$ \\
\hline NGC5055 & $247 \pm 017 \mathrm{E}+0$ & $140 \pm 009 \mathrm{E}+0$ & $516 \pm 036 \mathrm{E}+0$ & $628 \pm 044 \mathrm{E}+0$ & $389 \pm 039 \mathrm{E}-1$ \\
\hline NGC5194 & $288 \pm 020 \mathrm{E}+0$ & $177 \pm 012 \mathrm{E}+0$ & $977 \pm 069 \mathrm{E}+0$ & $\ldots$ & $149 \pm 015 E+0$ \\
\hline NGC5195 & $814 \pm 057 \mathrm{E}-1$ & $456 \pm 032 \mathrm{E}-1$ & $582 \pm 041 \mathrm{E}-1$ & $122 \pm 008 \mathrm{E}+0$ & $495 \pm 053 \mathrm{E}-2$ \\
\hline NGC5398 & $394 \pm 028 \mathrm{E}-2$ & $211 \pm 015 \mathrm{E}-2$ & $823 \pm 060 \mathrm{E}-2$ & $263 \pm 019 \mathrm{E}-1$ & $420 \pm 080 \mathrm{E}-3$ \\
\hline NGC5457 & $254 \pm 018 \mathrm{E}+0$ & $155 \pm 011 \mathrm{E}+0$ & $655 \pm 046 \mathrm{E}+0$ & $102 \pm 007 \mathrm{E}+1$ & $749 \pm 075 \mathrm{E}-1$ \\
\hline NGC5408 & $333 \pm 024 \mathrm{E}-2$ & $194 \pm 014 \mathrm{E}-2$ & $\cdots$ & $433 \pm 031 \mathrm{E}-1$ & $\ldots$ \\
\hline NGC5474 & $113 \pm 008 \mathrm{E}-1$ & $638 \pm 046 \mathrm{E}-2$ & $979 \pm 084 \mathrm{E}-2$ & $134 \pm 014 \mathrm{E}-1$ & $120 \pm 023 \mathrm{E}-2$ \\
\hline NGC5713 & $205 \pm 014 \mathrm{E}-1$ & $136 \pm 009 \mathrm{E}-1$ & $100 \pm 007 \mathrm{E}+0$ & $235 \pm 016 \mathrm{E}+0$ & $159 \pm 016 \mathrm{E}-1$ \\
\hline NGC5866 & $704 \pm 049 \mathrm{E}-1$ & $384 \pm 027 \mathrm{E}-1$ & $240 \pm 017 \mathrm{E}-1$ & $222 \pm 017 \mathrm{E}-1$ & $227 \pm 030 \mathrm{E}-2$ \\
\hline IC4710 & $602 \pm 043 \mathrm{E}-2$ & $349 \pm 027 \mathrm{E}-2$ & $\ldots$ & $\ldots$ & $\ldots$ \\
\hline NGC6822 & $160 \pm 011 \mathrm{E}+0$ & $873 \pm 065 \mathrm{E}-1$ & $139 \pm 015 \mathrm{E}+0$ & $286 \pm 034 \mathrm{E}+0$ & $694 \pm 140 \mathrm{E}-2$ \\
\hline NGC6946 & $310 \pm 022 \mathrm{E}+0$ & $202 \pm 014 \mathrm{E}+0$ & $122 \pm 008 \mathrm{E}+1$ & $205 \pm 014 \mathrm{E}+1$ & $139 \pm 014 \mathrm{E}+0$ \\
\hline NGC7331 & $163 \pm 011 \mathrm{E}+0$ & $984 \pm 069 \mathrm{E}-1$ & $335 \pm 023 \mathrm{E}+0$ & $429 \pm 030 \mathrm{E}+0$ & $372 \pm 037 \mathrm{E}-1$ \\
\hline NGC7552 & $440 \pm 031 \mathrm{E}-1$ & $347 \pm 024 \mathrm{E}-1$ & $270 \pm 019 \mathrm{E}+0$ & $114 \pm 008 \mathrm{E}+1$ & $275 \pm 028 \mathrm{E}-1$ \\
\hline NGC7793 & $775 \pm 054 \mathrm{E}-1$ & $468 \pm 033 \mathrm{E}-1$ & $150 \pm 010 \mathrm{E}+0$ & $188 \pm 013 \mathrm{E}+0$ & $102 \pm 009 \mathrm{E}-1$ \\
\hline
\end{tabular}

Note. The compact table entry format TUV \pm WXYEZ implies (T.UV \pm W.XY) $\times 10^{\mathrm{Z}}$ in Jy. Corrections for neither Galactic nor intrinsic extinction has been applied. The uncertainties include both statistical and systematic effects. No color corrections have been applied. The filter central wavelengths and widths are computed via Equations (1) and (2).

\section{Results}

\subsection{Flux Densities}

Table 2 presents the spatially integrated flux densities for all 79 SINGS+KINGFISH galaxies for 30 photometric bands. In Table 5 we also supply global aperture photometry for the few cases where upper limits are provided in Table 2. The tabulated flux densities include aperture corrections (Section 3.6) and are not corrected for Galactic extinction. No color corrections have been applied to the data in Table 2. Some of the fluxes presented here remain unchanged from the values published elsewhere, for example, 2MASS $J H K_{\mathrm{s}}$ Spitzer IRAC and MIPS, SCUBA $850 \mu \mathrm{m}$, and VLA $20 \mathrm{~cm}$ photometry. However, if any differences exist between values published in multiple publications, precedence is given to the more recent published values, e.g., 2MASS and Spitzer photometry from the Local Volume Legacy publication of Dale et al. (2009) is given priority over the 2MASS and Spitzer photometry appearing in Dale et al. (2007).

Figure 2 provides a comparison of our updated optical fluxes with those presented in previous publications. The updated $V R_{\mathrm{C}} I_{\mathrm{C}}$ optical fluxes are generally in agreement, on average, with previously published values, and the $1 \sigma$ scatters in the differences for these filters are $\sim 0.2-0.3 \mathrm{mag}$. The differences with published $B$ filter fluxes, however, show a locus of points indicating that the literature data are typically $0.2 \mathrm{mag}$ fainter than what we obtain after calibrating via Pan-STARRS1. There is also a second grouping of $B$ data points, comprising nearly one-third of the total sample, that indicates the Dale et al. (2007) data are $\sim 0.4$ mag brighter; these may be cases where the $B$ standard star calibration images for Dale et al. (2007) were either saturated or obtained in non-photometric conditions, both of which would lead to artificially faint standard star counts/s and thus artificially bright galaxy fluxes.

Figure 3 provides a similar comparison for the Herschel farinfrared/submillimeter photometry. The updated SPIRE beam sizes are larger than those used in Dale et al. (2012) by [11, 11, $14] \%$ at $[250,350,500] \mu \mathrm{m}$, which naturally leads to fainter SPIRE fluxes. This decrease is evident in Figure 3, where the average SPIRE flux is $\sim[8,5,16] \%$ fainter at $[250,350,500] \mu \mathrm{m}$ than what appeared in Dale et al. (2012). For the five galaxies where we incorporated additional PACS data from other observing programs (Holmberg II, IC 2574, NGC 2798, NGC 4236, and NGC 4631; see Section 3), the resulting PACS maps are deeper and thus allow for some diffuse flux to be additionally detected. For faint Holmberg II it makes an appreciable difference: the 70 and $100 \mu \mathrm{m}$ global fluxes are now $40 \%-50 \%$ larger.

\subsection{The Spectral Energy Distributions}

Figure 4 shows the observed infrared/submillimeter SEDs for the KINGFISH sample. Included in each panel, when available, are the GALEX far- and near-ultraviolet, $B V R_{\mathrm{C}} I_{\mathrm{C}}$ and ugriz optical, 2MASS $J H K_{\mathrm{s}}$ near-infrared, Spitzer 3.6, 4.5, 5.8, 8.0, 24, 70, and $160 \mu \mathrm{m}$, WISE $12 \mu \mathrm{m}$, Herschel 70, $100,160,250,350$, and $500 \mu \mathrm{m}$, and Planck and SCUBA $850 \mu \mathrm{m}$ fluxes.

The broadband SEDs displayed in Figure 4 are fitted with the models of Draine \& Li (2007) over the wavelength range 3.6-500 $\mu \mathrm{m}$, models based on mixtures of amorphous silicate and graphitic dust grains that effectively reproduce the average Milky Way extinction curve and are consistent with observations of PAH features and the variety of infrared continua in local galaxies. A total of four free parameters are used in the fits: the fraction $q_{\mathrm{PAH}}$ of the dust mass residing in polycyclic 
Table 5

Aperture Photometry for Cases of Upper Limits in Table 2

\begin{tabular}{|c|c|c|c|c|c|c|c|c|c|c|c|c|}
\hline $\begin{array}{l}\text { Filter } \\
\bar{\lambda}(\mu \mathrm{m})\end{array}$ & $\begin{array}{c}\text { Spitzer } \\
\text { IRAC } \\
5.76\end{array}$ & $\begin{array}{c}\text { Spitzer } \\
\text { IRAC } \\
7.96\end{array}$ & $\begin{array}{c}\text { WISE } \\
\text { W3 } \\
12.8\end{array}$ & $\begin{array}{c}\text { Spitzer } \\
\text { MIPS } \\
23.8\end{array}$ & $\begin{array}{c}\text { Spitzer } \\
\text { MIPS } \\
72.5\end{array}$ & $\begin{array}{c}\text { Herschel } \\
\text { PACS } \\
71.8\end{array}$ & $\begin{array}{c}\text { Herschel } \\
\text { PACS } \\
103\end{array}$ & $\begin{array}{c}\text { Spitzer } \\
\text { MIPS } \\
157\end{array}$ & $\begin{array}{c}\text { Herschel } \\
\text { PACS } \\
167\end{array}$ & $\begin{array}{c}\text { Herschel } \\
\text { SPIRE } \\
252\end{array}$ & $\begin{array}{c}\text { Herschel } \\
\text { SPIRE } \\
353\end{array}$ & $\begin{array}{c}\text { Herschel } \\
\text { SPIRE } \\
511\end{array}$ \\
\hline NGC0584 & $\ldots$ & $\ldots$ & $\ldots$ & $\ldots$ & & & & & & $281 \pm 158 \mathrm{E}-1$ & $110 \pm 106 \mathrm{E}-1$ & $099 \pm 684 \mathrm{E}-2$ \\
\hline NGC1404 & & & & & $110 \pm 080 \mathrm{E}-1$ & $330 \pm 062 \mathrm{E}-1$ & $-111 \pm 009 \mathrm{E}+0$ & $130 \pm 120 \mathrm{E}-1$ & $630 \pm 066 \mathrm{E}-1$ & $-149 \pm 030 \mathrm{E}-1$ & $-139 \pm 025 \mathrm{E}-1$ & $295 \pm 166 \mathrm{E}-2$ \\
\hline M81dwA & $-010 \pm 520 \mathrm{E}-3$ & $034 \pm 640 \mathrm{E}-3$ & $-420 \pm 090 \mathrm{E}-4$ & $050 \pm 330 \mathrm{E}-3$ & $029 \pm 146 \mathrm{E}-1$ & $330 \pm 090 \mathrm{E}-2$ & $\ldots$ & $020 \pm 473 \mathrm{E}-1$ & $\ldots$ & $\ldots$ & $\ldots$ & $\ldots$ \\
\hline DDO053 & & & & $\ldots$ & & & & & $\ldots$ & & & $980 \pm 578 \mathrm{E}-3$ \\
\hline HoIX & $-400 \pm 200 \mathrm{E}-4$ & $771 \pm 100 \mathrm{E}-3$ & $526 \pm 050 \mathrm{E}-3$ & $128 \pm 040 \mathrm{E}-3$ & $068 \pm 120 \mathrm{E}-2$ & $\ldots$ & $\ldots$ & $-251 \pm 260 \mathrm{E}-2$ & $\ldots$ & $\ldots$ & $\ldots$ & \\
\hline DDO154 & $560 \pm 080 \mathrm{E}-3$ & $180 \pm 200 \mathrm{E}-4$ & $422 \pm 035 \mathrm{E}-3$ & $145 \pm 050 \mathrm{E}-3$ & $338 \pm 140 \mathrm{E}-2$ & $-270 \pm 019 \mathrm{E}-1$ & $500 \pm 029 \mathrm{E}-1$ & $-009 \pm 280 \mathrm{E}-2$ & $600 \pm 136 \mathrm{E}-2$ & $109 \pm 011 \mathrm{E}-1$ & $693 \pm 093 \mathrm{E}-2$ & $294 \pm 061 \mathrm{E}-2$ \\
\hline DDO165 & $-000-5$ & $100-2000$ & $800 \pm 280 \mathrm{E}-4$ & $449 \pm 060 \mathrm{E}-3$ & $141 \pm 020 \mathrm{E}-1$ & $-240 \pm 021 \mathrm{E}-1$ & $150 \pm 022 \mathrm{E}-1$ & $206 \pm 043 \mathrm{E}-1$ & $400 \pm 178 \mathrm{E}-2$ & $101 \pm 133 \mathrm{E}-2$ & $100 \pm 124 \mathrm{E}-2$ & $501 \pm 092 \mathrm{E}-2$ \\
\hline
\end{tabular}

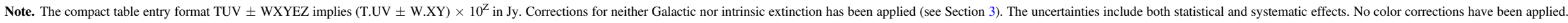




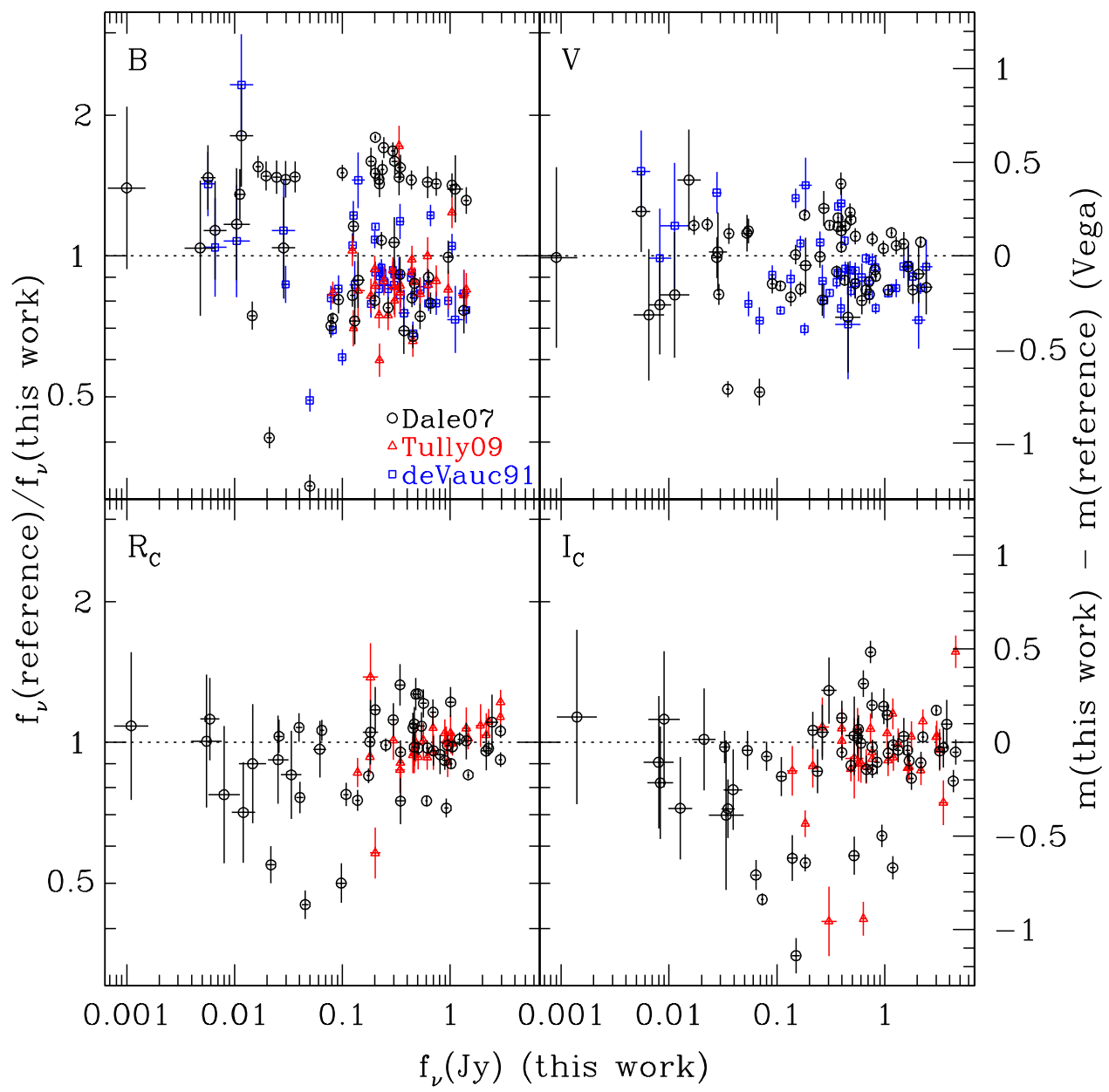

Figure 2. Comparison of global $B V R_{\mathrm{C}} I_{\mathrm{C}}$ galaxy photometry from the literature with those measured here that are calibrated based on Pan-STARRS1 $g_{\mathrm{P} 1} r_{\mathrm{P} 1} i_{\mathrm{P} 1} z_{\mathrm{P} 1}$ photometry on field stars (see Section 3.1).

aromatic hydrocarbons (PAHs), the intensity $U_{\min }$ of the interstellar radiation field that heats the general diffuse interstellar medium, and the fraction $\gamma$ of the dust mass heated by more intense starlight distributions such as those arising from photodissociation regions (PDRs) in star-forming regions, the ratio of the stellar mass to the dust mass ( $M_{\text {dust }}$ is determined by the normalization of the SED model with the observed photometric data). The parameter $q_{\mathrm{PAH}}$ ranges between $0 \%$ and $12 \%$, and $U_{\min }$ can have values between 0.01 and 30. As was done for Draine et al. (2007) and Dale et al. (2012), we minimize the number of free parameters by fixing the maximum value of the interstellar radiation field $\left(U_{\max }=10^{6}\right)$ as well as the power-law exponent that governs the distribution of starlight intensities heating the dust $(\alpha=2)$. More details of these models may be found in Draine \& $\mathrm{Li}$ (2007) and Draine et al. (2007).

The new SED fits here can be compared to what was obtained previously for the KINGFISH sample in Dale et al. (2012) with the outdated SPIRE photometric calibration. Figure 5 provides such a comparison for dust mass, PAH fraction, and the properties of the radiation field that is heating the dust. The two systematic differences, in the inferred dust mass and the diffuse radiation field intensity $U_{\min }$, are the result of the changes in the PACS and SPIRE calibrations-brighter at 70 and $100 \mu \mathrm{m}$ and fainter at 250,350 , and $500 \mu \mathrm{m}$ result in warmer interstellar dust and smaller overall dust masses. We note that while recent results indicate a necessary change in the DL07 dust opacities (Dalcanton et al. 2015; Planck Collaboration et al. 2016b), we are consistently using the same (original) dust models from Draine \& $\mathrm{Li}$ (2007) in the Figure 5 comparisons. The two outliers in $q_{\mathrm{PAH}}$ are the result of the inclusion of new PACS photometry for NGC 0584 and an improved sky estimate for NGC 1291's PACS $70 \mu \mathrm{m}$ map, which led to a factor of $\sim 2$ smaller flux that is now in much better agreement with the Spitzer $70 \mu \mathrm{m}$ flux.

As was mentioned in Dale et al. (2012), the faintest targets pose the most challenges to SED fitting. Spatial variations in the foreground cirrus amplify the relative uncertainty in the extracted fluxes for these targets. Even with our updated data processing and analysis, this situation remains essentially unchanged for galaxies like NGC 0584, DDO 053, M81 dwarf B, and Holmberg I. However, the addition of WISE fluxes to the SED fitting incrementally improves our confidence in the SED fits. A more detailed analysis of theoretical fits to these SEDs is presented in the companion paper (L.K. Hunt et al. 2017, in preparation) and in B.T. Draine et al. (2017, in preparation).

\subsection{Submillimeter Excess}

Dale et al. (2012) found an excess of submillimeter emission for several galaxies in the KINGFISH sample, where the observed $500 \mu \mathrm{m}$ emission was measured to lie significantly 


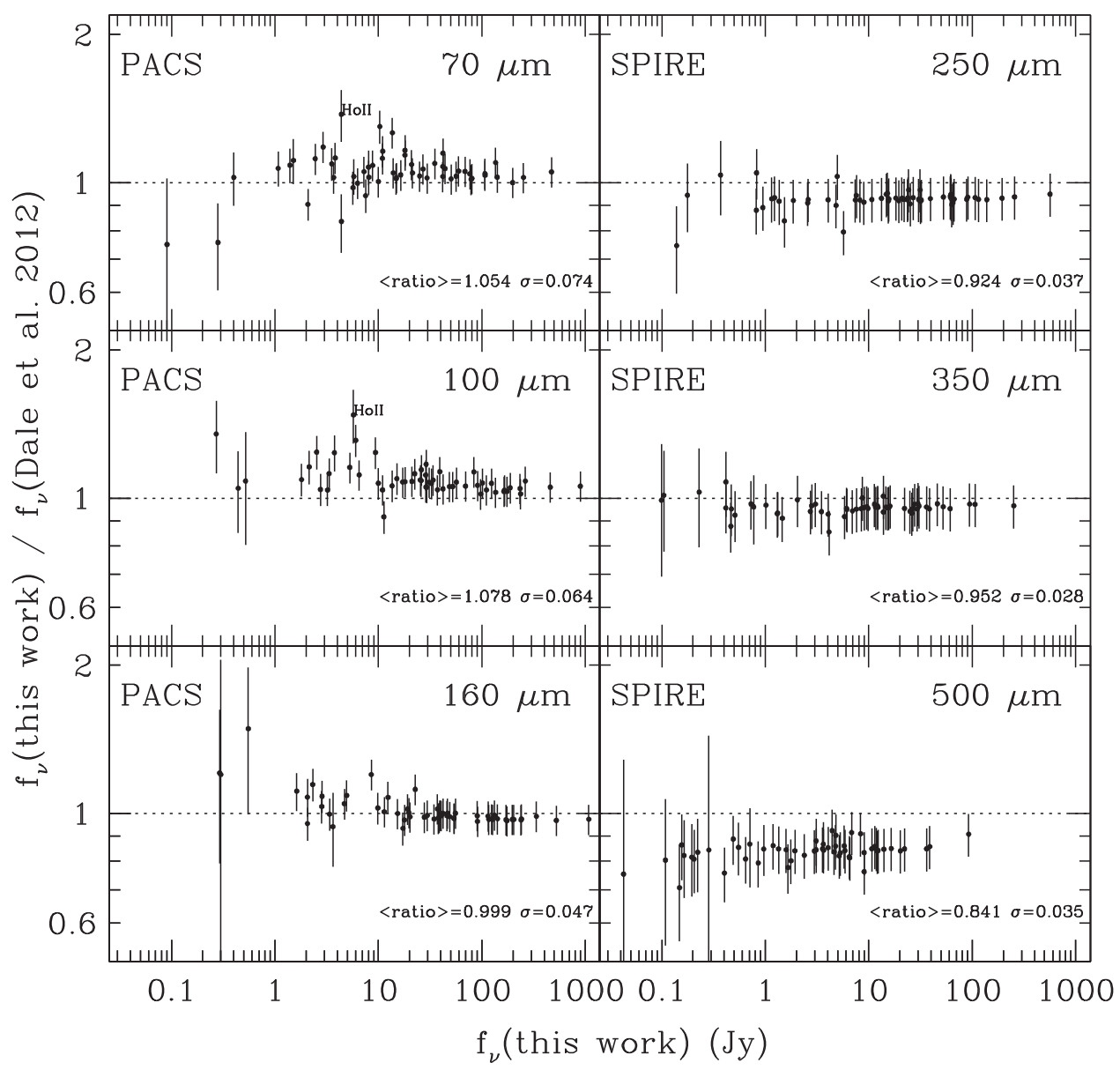

Figure 3. Comparison of updated global Herschel photometry with those presented in Dale et al. (2012). The error bars are relatively constant since they are primarily dominated by systematics for the brighter sources.

above the model predictions based on Draine \& Li (2007) fits to the observed 3.6-500 $\mu \mathrm{m}$ SEDs. The submillimeter excess $\xi$ $(500 \mu \mathrm{m})$ was quantitatively defined in Dale et al. (2012) as

$$
\xi(500 \mu \mathrm{m})=\frac{f_{\nu}(500 \mu \mathrm{m})_{\text {observed }}-f_{\nu}(500 \mu \mathrm{m})_{\text {model }}}{f_{\nu}(500 \mu \mathrm{m})_{\text {model }}} .
$$

This excess was primarily found in low-metallicity galaxies: 9 of the 10 dwarf/irregular/Magellanic galaxies in the KINGFISH sample with $500 \mu \mathrm{m}$ detections exhibited $\xi(500 \mu \mathrm{m})>0.60$. This result echoed similar claims of submillimeter excess in studies of M33, the Magellanic Clouds, and other lowmetallicity star-forming galaxies (Galliano et al. 2005; Galametz et al. 2011; Planck Collaboration et al. 2011; Gordon et al. 2014; Izotov et al. 2014; Hermelo et al. 2016). With the updated calibrations for our present data set, including SPIRE calibration changes that result in $500 \mu \mathrm{m}$ fluxes lower by an average of 16\% (Figure 3), we still see submillimeter excesses in the sample, but at lower significance. The average excess for dwarf/ irregular/Magellanic galaxies in Dale et al. (2012) was $\langle\xi(500 \mu \mathrm{m})\rangle_{T=\mathrm{Im}, \mathrm{I}, \mathrm{Sm}} \sim 0.70$, whereas in the current work the average value is $\langle\xi(500 \mu \mathrm{m})\rangle_{T=\mathrm{Im}, \mathrm{I}, \mathrm{Sm}} \sim 0.53$ for the 11 SINGS/KINGFISH dwarf/irregular/Magellanic galaxies with secure $500 \mu \mathrm{m}$ detections. Note that an alternative signal-tonoise-ratio-like definition of the submillimeter excess may be defined by normalizing via the model and observational uncertainties, namely

$$
\begin{aligned}
& \mathrm{S} / \mathrm{N}(500 \mu \mathrm{m} \text { excess }) \\
& =\frac{f_{\nu}(500 \mu \mathrm{m})_{\mathrm{observed}}-f_{\nu}(500 \mu \mathrm{m})_{\text {model }}}{\sqrt{\epsilon_{\text {obs }}^{2}+\epsilon_{\text {mod }}^{2}}} .
\end{aligned}
$$

In this case, the average value is $\langle\mathrm{S} / \mathrm{N}(500 \mu \mathrm{m}$ excess $)\rangle \sim 4.3$ for $\epsilon_{\mathrm{mod}}=0$ and $\sim 3.3$ for $\epsilon_{\mathrm{mod}}=0.1 f_{\nu}(500 \mu \mathrm{m})_{\text {model }}$ for the 11 SINGS/KINGFISH dwarf galaxies. We caution that our results may be biased since we restrict our analysis to securely detected sources.

Although the left-hand panel of Figure 6 shows no dependence for submillimeter excess on far-infrared color, there is a clear trend in the right-hand panel, with higher submillimeter excesses for lower gas-phase metallicity. This persistency of a submillimeter excess in primarily low-metallicity galaxies contradicts the analysis of Kirkpatrick et al. (2013), who found no significant submillimeter excesses for a subset of 20 galaxies from the KINGFISH sample with robust (and updated) far-infrared photometry that span a range in metallicity. However, there are three main differences between the analysis carried out here and by Kirkpatrick et al. (2013). First, Kirkpatrick et al. (2013) only fit data spanning $24-350 \mu \mathrm{m}$. Our SED fitting employs the full infrared continuum over 3.6-500 $\mu \mathrm{m}$, and therefore our approach must balance contributions from stellar and PAH emission in addition to that from larger dust grains. Second, Kirkpatrick et al. (2013) employ the superposition of two modified blackbodies, 


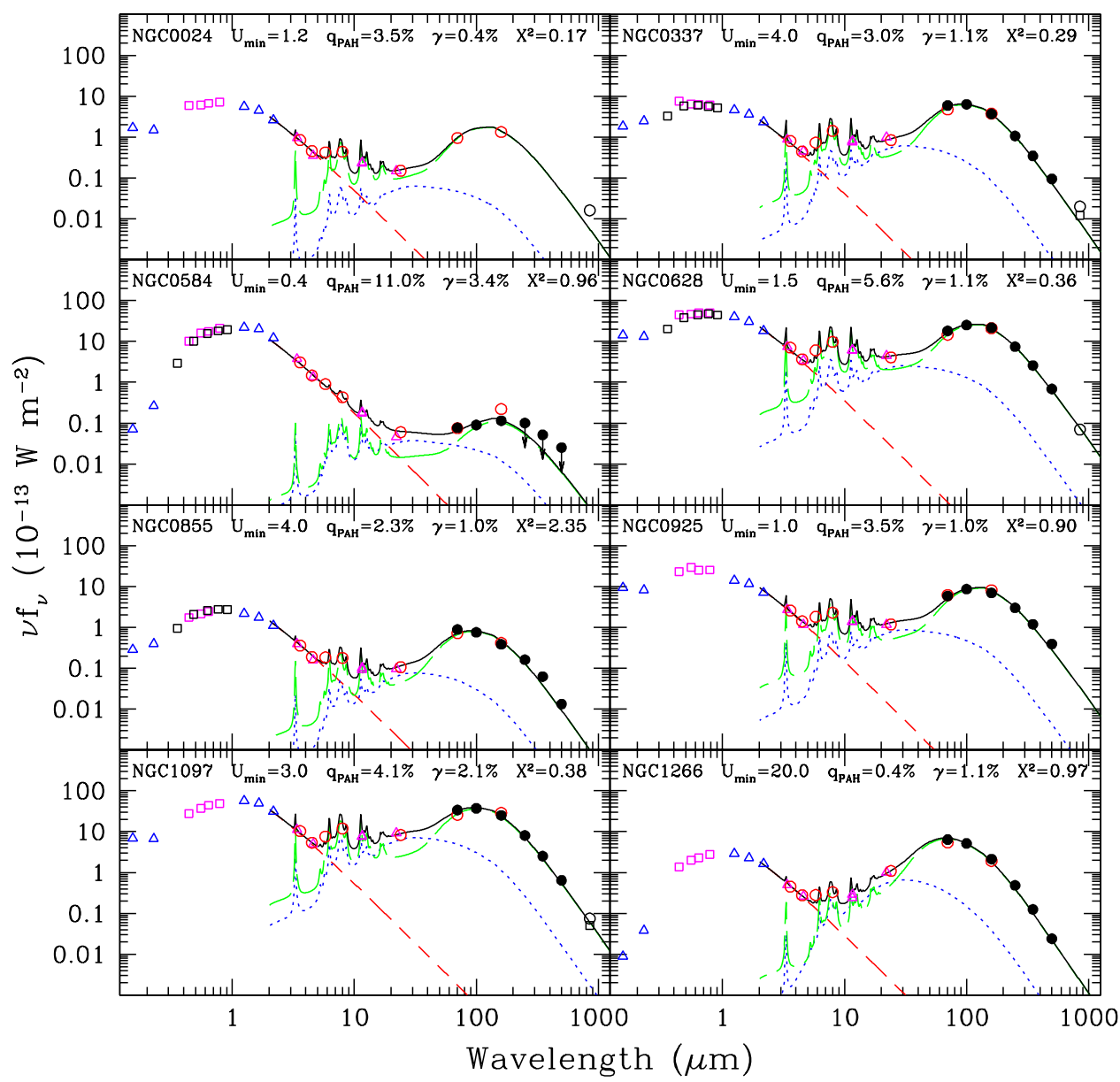

Figure 4. Globally integrated infrared/submillimeter spectral energy distributions for all the galaxies in the KINGFISH/SINGS sample, sorted by R.A. The following symbols are used: filled circles (Herschel), triangles (GALEX, 2MASS, and WISE), open circles (Spitzer and Planck), and squares (BVR $R_{\mathrm{C}} I_{\mathrm{C}}$ and $u g r i z$ and SCUBA). Arrows indicate $5 \sigma$ upper limits (and lower limits in the case of NGC 3034). The solid curve is the sum of a $5000 \mathrm{~K}$ stellar blackbody (short dashed) along with models of dust emission from PDRs (dotted; $U>U_{\mathrm{min}}$ ) and the diffuse interstellar medium (long dashed; $U=U_{\mathrm{min}}$ ). The fitted parameters from these Draine \& $\mathrm{Li}$ (2007) 3.6-500 $\mu \mathrm{m}$ model fits are listed within each panel along with the reduced $\chi^{2}$ (see Section 4.2 for details). While the plotted data are corrected for Galactic extinction, the fluxes tabulated in Table 2 are not corrected. The uncertainties are smaller than the symbols plotted. There are no SED fits in cases where the far-infrared photometry provides only limits.

(An extended version of this figure is available.)

whereas our fits use a more sophisticated dust model (Draine \& Li 2007); we have four free parameters in our SED fitting, whereas Kirkpatrick et al. (2013) have only three free parameters: the temperature and emissivity of the cold dust component, and the ratio of the amplitudes of the two modified blackbodies. Third, while we normalize our submillimeter excesses by the predicted model value at $500 \mu \mathrm{m}$, Kirkpatrick et al. (2013) normalize via the observed $500 \mu \mathrm{m}$ value. Normalizing by the observed flux is an approach that naturally depresses the excess measure. For example, the average submillimeter excess for the 11 SINGS/ KINGFISH dwarf/irregular/Magellanic galaxies with $500 \mu \mathrm{m}$ detections is 0.33 if the normalization is via the observed flux, a factor of 1.6 times smaller than when normalizing by the predicted model flux.

\section{Summary}

We present an update to the full panchromatic photometric database for the 79 galaxies in the combined SINGS/KINGFISH sample of nearby galaxies, for ultraviolet through radio wavelengths. Updates include incorporating recent improvements in the calibration of the Herschel photometers and a recalibration of $B V R_{\mathrm{C}} I_{\mathrm{C}}$ photometry using broadband data from the PanSTARRS1 survey. On average, the updated Herschel fluxes differ by $[+5,+8,0,-8,-5,-16] \%$ at $[70,100,160,250,350$, 500] $\mu \mathrm{m}$ compared to what was published in the original KINGFISH photometry paper of Dale et al. (2012). The average updated fluxes for the $V R_{\mathrm{C}} I_{\mathrm{C}}$ filters are essentially unchanged from the SINGS photometry presented in Dale et al. (2007), but with a scatter of $0.2-0.3 \mathrm{mag}$. The updated collection of $B$ fluxes are about $\sim 0.2 \mathrm{mag}$ brighter than what appears in the literature. Finally, theoretical Draine \& Li (2007) SED models are fit to each galaxy's 3.6-500 $\mu \mathrm{m}$ data set. Two of the fitted parameters show small but systematic differences with the results published previously: the total dust masses are about $20 \%$ lower, and the typical value of the radiation field that is heating the diffuse ISM is about $10 \%$ higher. Both of these results naturally arise from the changes in the calibrations of the Herschel imagers since 2012. We confirm our previous finding of an excess of submillimeter emission $(500 \mu \mathrm{m})$ in primarily low-metallicity dwarf/irregular galaxies, but at a smaller amplitude due to the updated calibration of the Herschel SPIRE beams. A full exploration of this panchromatic data set is carried out in a companion paper by $\mathrm{L}$. K. Hunt et al. (2017, in preparation).

We thank the referee for excellent suggestions that improved this work. Herschel is an ESA space observatory with science 

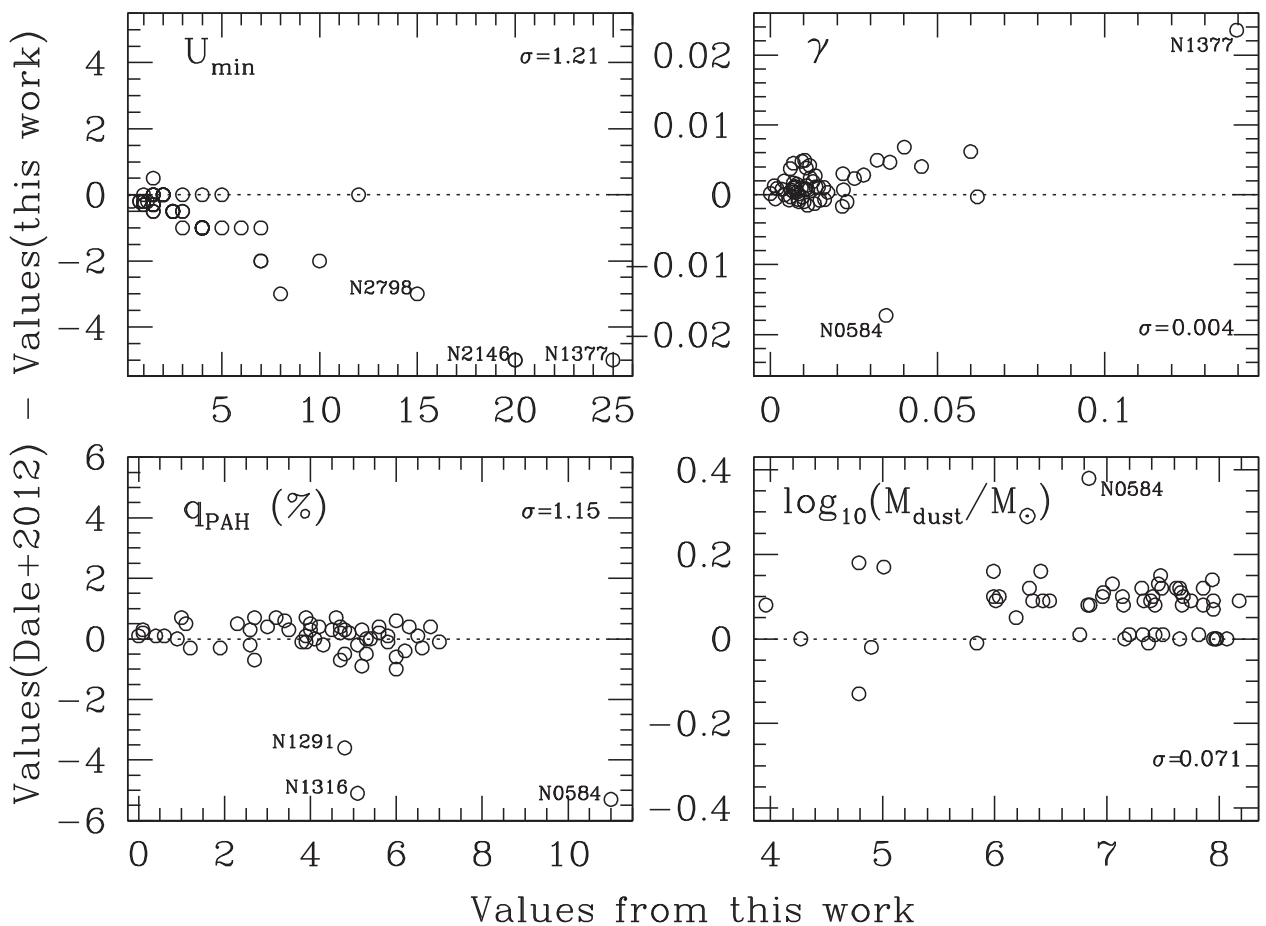

Figure 5. Comparison of DL07 output fit parameters from Dale et al. (2012) with those measured here. The dispersions in the ordinate (y-axis) values are inset.

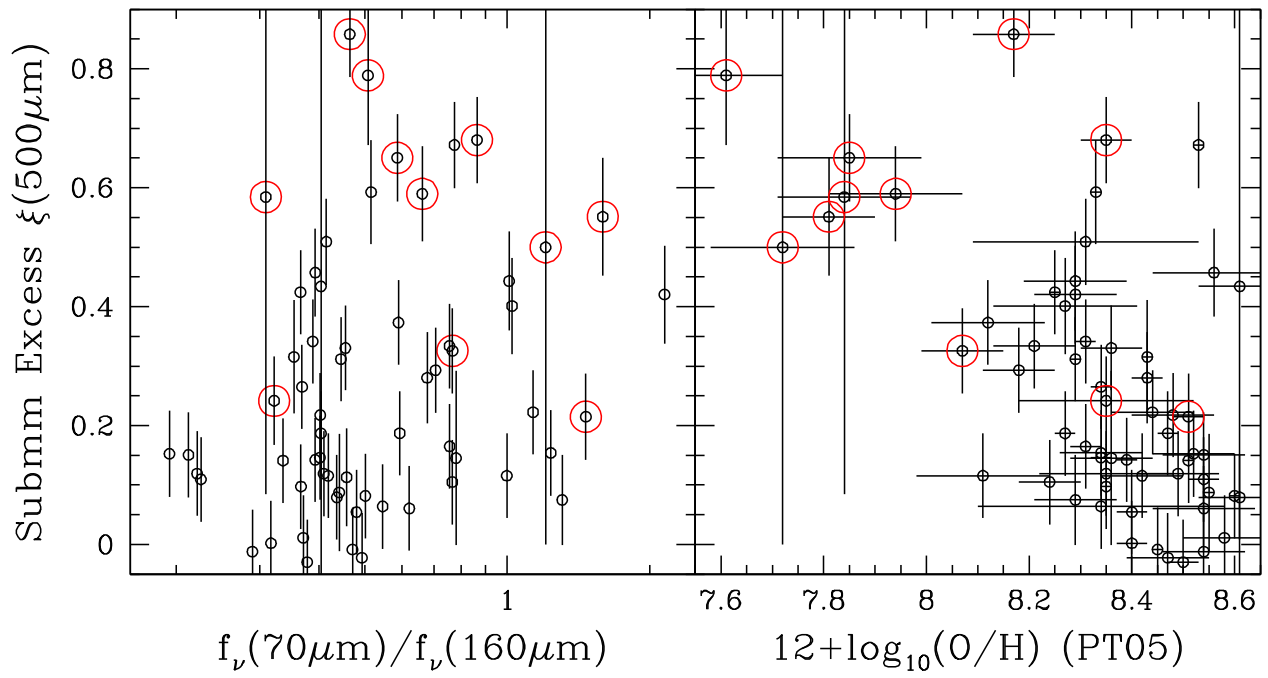

Figure 6. The submillimeter excess parameter $\xi(500 \mu \mathrm{m})$ (see Equation (7)) as a function of far-infrared color and characteristic oxygen abundance as derived from Moustakas et al. (2010). Red circles indicate irregular galaxies $(T=\mathrm{Sm}$, Im, or I0).

instruments provided by European-led Principal Investigator consortia and with important participation from NASA. This work is based on observations made with the Spitzer Space Telescope and utilizes the NASA/IPAC Infrared Science Archive, both operated by JPL/Caltech under a contract with NASA. We gratefully acknowledge NASA's support for construction, operation, and science analysis for the GALEX mission, developed in cooperation with the Centre National d'Etudes Spatiales of France and the Korean Ministry of Science and Technology. Funding for the Sloan Digital Sky Survey and SDSS-II has been provided by the Alfred P. Sloan Foundation, the Participating Institutions, the NSF, the U.S. Department of Energy, NASA, the Japanese Monbukagakusho, the Max Planck Society, and the Higher Education Funding
Council for England. This publication makes use of data products from the Wide-field Infrared Survey Explorer, which is a joint project of the University of California, Los Angeles, and the JPL/Caltech, funded by NASA. The Pan-STARRS1 Surveys have been made possible through contributions of the Institute for Astronomy, the University of Hawaii, the PanSTARRS Project Office, the Max Planck Society and its participating institutes, the Max Planck Institute for Astronomy, Heidelberg and the Max Planck Institute for Extraterrestrial Physics, Garching, The Johns Hopkins University, Durham University, the University of Edinburgh, Queen's University Belfast, the Harvard-Smithsonian Center for Astrophysics, the Las Cumbres Observatory Global Telescope Network Incorporated, the National Central University of 


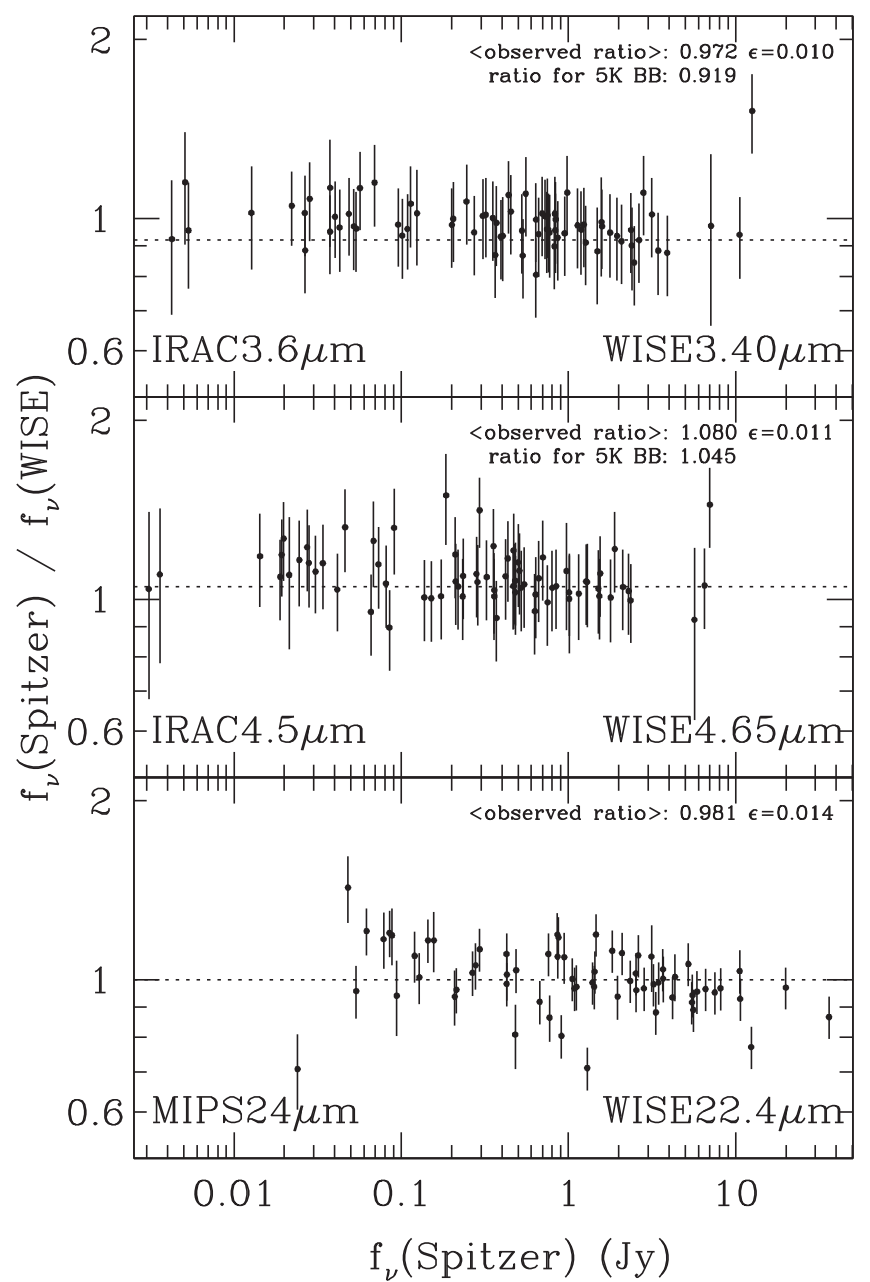

Figure 7. Comparison of Spitzer- and aperture-based global photometry with total WISE photometry. The dotted lines for the top two panels indicate the expected flux density ratios after convolving a $5000 \mathrm{~K}$ blackbody with the respective filter bandpass profiles. The average ratio and its uncertainty $(\sigma / \sqrt{N})$ are inset.

Taiwan, the Space Telescope Science Institute, the NSF, the University of Maryland, and Eotvos Lorand University and the Los Alamos National Laboratory.

\section{Appendix WISE Fluxes}

Table 2 presents WISE W3 $(12 \mu \mathrm{m})$ flux densities based on the same apertures used for the other flux densities presented in Table 2. We additionally present here "total" flux densities for all four WISE bands using zero-points of [309.666, 170.623, $29.043,7.875] \mathrm{Jy}$ for [W1, W2, W3, W4] at central wavelengths of $[3.4,4.6,12,22] \mu \mathrm{m}^{26}$ The process for extracting "total" flux densities follows that described in Jarrett et al. (2013), whereby azimuthally averaged elliptical surface brightness profiles are extrapolated to three disk scale lengths beyond the $1 \sigma$ (sky rms) isophotal radii. For WISE, the $1 \sigma$ isophotes are at surface brightness levels of approximately $[23.0,21.8,18.1,15.8] \mathrm{mag} \operatorname{arcsec}^{-2}$ (Vega) for [W1, W2, W3, W4] (Jarrett et al. 2013). This particular WISE database provides an excellent opportunity to check our Spitzer- and aperture-based global fluxes at 3.6, 4.5, and $24 \mu \mathrm{m}$. Figure 7 presents such a comparison. The agreement between Spitzer and WISE flux densities is close to expectations. The dotted lines in the top two panels indicate the expected flux density ratios after convolving the filter bandpasses with a $5000 \mathrm{~K}$ stellar blackbody; the observed ratios match the expected ratios to within the observed $1 \sigma$ scatters. There is perhaps a weak trend for the ratio of Spitzer $24 \mu \mathrm{m}$ and WISE W3, with the ratio decreasing with increasing brightness.

\section{References}

Bendo, G. J., Wilson, C. D., Pohlen, M., et al. 2010, A\&A, 518, L65 Blanton, M. R., Schlegel, D. J., Strauss, M. A., et al. 2005, AJ, 129, 2562 Brown, M. J. I., Jarrett, T. H., \& Cluver, M. E. 2014, PASA, 31, 49 Chonis, T. S., \& Gaskell, C. M. 2008, AJ, 135, 264

Cook, D. O., Dale, D. A., Johnson, B. D., et al. 2014, MNRAS, 445, 881 da Cunha, E., Charlot, S., \& Elbaz, D. 2008, MNRAS, 388, 1595 Dalcanton, J. J., Fouesneau, M., Hogg, D. W., et al. 2015, ApJ, 814, 3 Dale, D. A., Aniano, G., Engelbracht, C. W., et al. 2012, ApJ, 745, 95 Dale, D. A., Bendo, G. J., Engelbracht, C. W., et al. 2005, ApJ, 633, 857 Dale, D. A., Cohen, S. A., Johnson, L. C., et al. 2009, ApJ, 703, 517 Dale, D. A., Gil de Paz, A., Gordon, K. D., et al. 2007, ApJ, 655, 863 Dale, D. A., Helou, G., Magdis, G. E., et al. 2014, ApJ, 784, 83 Dale, D. A., Smith, J. D. T., Armus, L., et al. 2006, ApJ, 646, 161 de Vaucouleurs, G., de Vaucouleurs, A., Corwin, H. G., Jr., et al. 1991, Third Reference Catalogue of Bright Galaxies (New York: Springer) Draine, B. T., Dale, D. A., Bendo, G., et al. 2007, ApJ, 663, 866 Draine, B. T., \& Li, A. 2007, ApJ, 657, 810

Galametz, M., Madden, S. C., Galliano, F., et al. 2011, A\&A, 532, A56 Galliano, F., Madden, S. C., Jones, A. P., Wilson, C. D., \& Bernard, J.-P. 2005, A\&A, 434, 867

Gordon, K. D., Roman-Duval, J., Bot, C., et al. 2014, ApJ, 797, 85 Grier, C. J., Mathur, S., Ghosh, H., \& Ferrarese, L. 2011, ApJ, 731, 60 Hermelo, I., Relaño, M., Lisenfeld, U., et al. 2016, A\&A, 590, A56 Izotov, Y. I., Guseva, N. G., Fricke, K. J., Krügel, E., \& Henkel, C. 2014, A\&A, 570, A97

Jarrett, T. H., Masci, F., Tsai, C. W., et al. 2013, AJ, 145, 6

Jester, S., Schneider, D. P., Richards, G. T., et al. 2005, AJ, 130, 873 Jonsson, P., Groves, B. A., \& Cox, T. J. 2010, MNRAS, 403, 17 Jordi, K., Grebel, E. K., \& Ammon, K. 2006, A\&A, 460, 339 Kartaltepe, J. S., Sanders, D. B., Le Floc'h, E., et al. 2010, ApJ, 709, 572 Kennicutt, R. C., Calzetti, D., Aniano, G., et al. 2011, PASP, 123, 1347 Kennicutt, R. C., Jr., Armus, L., Bendo, G., et al. 2003, PASP, 115, 928 Kirkpatrick, A., Calzetti, D., Galametz, M., et al. 2013, ApJ, 778, 51 Lupton, R. H., Jurić, M., Ivezić, Z., et al. 2005, BAAS, 37, 1384 Magnier, E. A., Schlafly, E., Finkbeiner, D., et al. 2013, ApJS, 205, 20 Maiolino, R., Carniani, S., Fontana, A., et al. 2015, MNRAS, 452, 54 Moustakas, J., Kennicutt, R. C., Jr., Tremonti, C. A., et al. 2010, ApJS, 190, 233

Muñoz-Mateos, J. C., Gil de Paz, A., Zamorano, J., et al. 2009, ApJ, 703, 1569 Noll, S., Burgarella, D., Giovannoli, E., et al. 2009, A\&A, 507, 1793

Planck Collaboration, Ade, P. A. R., Aghanim, N., et al. 2011, A\&A, 536, A17 Planck Collaboration, Ade, P. A. R., Aghanim, N., et al. 2016a, A\&A, 594, A26

Planck Collaboration, Ade, P. A. R., Aghanim, N., et al. 2016b, A\&A, 586, A132

Rémy-Ruyer, A., Madden, S. C., Galliano, F., et al. 2015, A\&A, 582, A121 Roussel, H. 2013, PASP, 125, 1126

Schlafly, E. F., \& Finkbeiner, D. P. 2011, ApJ, 737, 103

Schlafly, E. F., Finkbeiner, D. P., Jurić, M., et al. 2012, ApJ, 756, 158

Scolnic, D., Casertano, S., Riess, A., et al. 2015, ApJ, 815, 117

Scoville, N., Sheth, K., Aussel, H., et al. 2016, ApJ, 820, 83

Silva, L., Granato, G. L., Bressan, A., \& Danese, L. 1998, ApJ, 509, 103

Smith, J. D. T., Draine, B. T., Dale, D. A., et al. 2007, ApJ, 656, 770

Tajer, M., Trinchieri, G., Wolter, A., et al. 2005, A\&A, 435, 799

Tonry, J. L., Stubbs, C. W., Lykke, K. R., et al. 2012, ApJ, 750, 99

Tully, R. B., Rizzi, L., Shaya, E. J., et al. 2009, AJ, 138, 323

Wright, E. L., Eisenhardt, P. R. M., Mainzer, A. K., et al. 2010, AJ, 140, 1868

26 Brown et al. (2014) suggest W4 has an effective central wavelength of $22.8 \mu \mathrm{m}$. 